GABRIEL DO NASCIMENTO GUIMARÃES

A GEOID MODEL IN THE STATE OF SÃO PAULO: AN ATTEMPT FOR THE EVALUATION OF DIFFERENT METHODOLOGIES 
GABRIEL DO NASCIMENTO GUIMARÃES

\section{A GEOID MODEL IN THE STATE OF SÃO PAULO: AN ATTEMPT FOR THE EVALUATION OF DIFFERENT METHODOLOGIES}

Thesis presented to the Polytechnic School of the University of São Paulo of the requirements for the Degree of Doctor of Philosophy

Area of Concentration:

Transportation Engineering

Emphasis:

Spatial Information

Brazilian Supervisor:

Prof. Denizar Blitzkow

Italian Supervisor:

Prof. Riccardo Barzaghi 
Este exemplar foi revisado e alterado em relação à versão original, sob responsabilidade única do autor e com a anuência de seu orientador.

São Paulo, 28 de fevereiro de 2013.

Assinatura do autor

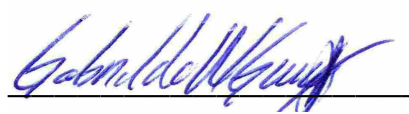

Assinatura do orientador

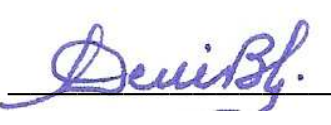

FICHA CATALOGRÁFICA

Guimarães, Gabriel do Nascimento

A geoid model in the state of São Paulo: an attempt for the evaluation of different methodologies / G. N. Guimarães. -ed. rev. -- São Paulo, 2013.

$144 \mathrm{p}$.

Tese (Doutorado) - Escola Politécnica da Universidade de São Paulo. Departamento de Engenharia de Transportes.

1. Geodésia física 2. Gravidade 3. Satélites artificiais I. Uni versidade de São Paulo. Escola Politécnica. Departamento de Engenharia de Transportes II. t. 
This thesis is dedicated to my brother Pedro Henrique (in memorian) and my best friend Arthur (in memorian). 


\section{ACKNOWLEDGEMENTS}

I thank God above all, by discernment, wisdom and health given to me, to solve, to circumvent and overcome all difficulties encountered along this route.

I would like to express my appreciation and thank my Brazilian supervisor, Dr. Denizar Blitzkow for his support and guidance throughout my studies, and for his friendship. His continuous encouragement and advice were greatly appreciated.

I also would like to thank my Italian supervisor, Dr. Riccardo Barzaghi for his opportunity to receive me as a research fellow in Italy at Politecnico di Milano during one year. It was an excellent occasion and an inspiring place to work.

To my father and mother for their support, dedication and guidance at all times. I could not have done it without them.

My colleague and talented researcher Dr. Ana Cristina de Oliveira Matos Cancoro, who helped me throughout this work.

Prof. Edvaldo Simões da Fonseca Junior and Prof. Ana Paula Larocca, for their support and valuable suggestions offered.

To my Brazilian colleagues Wagner, Flávio, Sônia, Augusto and Fernando. To my Italian colleagues Carlo, Noemi, Cynthia, Lucio, Cinzia and Elena.

To all employers of the Transportation Engineering Department of the University of São Paulo.

Finally, to the Brazilian government agencies (CAPES and CNPq) for the granting of Ph.D. scholarship. 
"Good decisions come from experience.

Experience comes from bad decisions."

Albert Einstein 


\begin{abstract}
The purpose of this thesis is to compute and to evaluate the geoid model in the State of São Paulo from two methodologies (Stokes' integral through the Fast Fourier Transform - FFT and Least Squares Collocation - LSC). Another objective of this study is to verify the potentiality of GOCE-based. Therefore, a brief study about mathematical foundations and fundamentals of Physical Geodesy is carried out. Some features of the Global Geopotential Models (GGMs) are discussed, as well as an overview of the new gravimetric missions. A special attention is given to GOCE mission. The theory related to Stokes' integral and Least Squares Collocation is also discussed in this work. The spectral decomposition was employed in the geoid models computation and the long wavelength component was represented by EGM2008 up to degree and order 150 and 360 and GOCE-based models up to 150. The models were compared in terms of geoid height residual and absolute and relative comparisons from GPS/leveling and the results show consistency between them. Also, a comparison in the mountain regions was carried out to verify the methodologies behavior in this area; the results showed that LSC is less consistent than FFT. Regarding GOCE-based models, 13 were tested, besides EGM2008. The evaluation was performed in terms of geoid height comparison obtained by GGMs over GPS/leveling and in terms of gravity disturbance. The evaluation shows that DIR _R3 and TIM_R3 presented more compatible results. The reason for the choice of São Paulo state is that there are a lot of geodetic activities and important engineering works that require the use of a height system. Furthermore, there are a lot of gravity and GPS/leveling data all around the state.
\end{abstract}

Keywords: Geodesy. GOCE. Geoid. Evaluation. 


\section{RESUMO}

Esta tese tem como propósito o cálculo e a avaliação do modelo geoidal no Estado de São Paulo a partir da aplicação de duas metodologias (integral de Stokes por meio da Transformada Rápida de Fourier - FFT e a colocação por mínimos quadrados - Least Squares Collocation - LSC). Outro objetivo deste trabalho é verificar a potencialidade dos mais recentes Modelos Globais do Geopotential (MGGs) baseados nos dados do satélite GOCE. Para tanto, um breve estudo é realizado sobre os fundamentos matemáticos e os da Geodésia Física. Algumas características dos MGGs são discutidas, bem como uma visão global das novas missões gravimétricas. Uma atenção especial é dada a missão do satélite GOCE. A teoria referente à integral de Stokes e a colocação por mínimos quadrados são outros temas discutidos no trabalho. A decomposição espectral foi empregada no cálculo dos modelos geoidais e a componente de longo comprimento de onda foi representada pelo modelo EGM2008 até grau e ordem 150 e 360 e aqueles baseados na missão GOCE até 150. Os modelos foram comparados entre si em termos do resíduo da altura geoidal e na forma absoluta e relativa por meio das estações GPS/RN. Os resultados apontaram consistência entre os modelos em termos de diferença média quadrática. Também foi realizado um estudo na região montanhosa a fim de verificar o comportamento das metodologias; os resultados mostraram que a LSC é menos consistente do que a FFT. No que diz respeito aos modelos baseados na missão GOCE, 13 foram testados, além do EGM2008. A avaliação foi realizada em termos da comparação da altura geoidal obtidas pelos MGGs com as estações GPS/nivelamento e em termos do distúrbio de gravidade. A avaliação mostra que os modelos DIR_R3 e TIM_R3 apresentaram os resultados mais compatíveis. A escolha do estado de São Paulo está relacionada à grande quantidade de trabalhos geodésicos e atividades na área da engenharia e que necessitam da utilização de um sistema altimétrico. Além disso, a grande quantidade de dados gravimétricos e de estações GPS/RN é mais uma justificativa para a realização do trabalho.

Palavras chaves: Geodésia. GOCE. Geoide. Avaliação. 


\section{LIST OF FIGURES}

Figure 2.1 Relationship between the adjustments issues............... 31

Figure 2.2 Original and linearized function........................................ 33

Figure 3.1 The components of the gravitational force................... 37

Figure 3.2 Geoid and ellipsoid surfaces........................................ 47

Figure 3.3 Spherical and rectangular coordinates.............................. 48

Figure $3.4 \quad$ Types of spherical harmonics......................................... 52

Figure $3.5 \quad$ Geodetic quantities................................................. 52

Figure 4.1 Science applications areas using GOCE data............... 59

Figure 4.2 The Electrostatic Gravity Gradiometer.............................. 60

Figure 4.3 Time series of gravity gradients (upper right). Gravity gradient maps (lower right)................................... 61

Figure 4.4 Degree variance (upper) and error degree variances

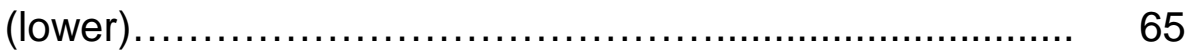

Figure 4.5 Amplitude and commission error........................... 66

Figure 4.6 Geoid height components................................ 70

Figure 4.7 Quantities involved in real and Helmert's spaces........... 78

Figure $4.8 \quad$ The basic model......................................... 83

Figure $4.9 \quad$ The planar grid............................................ 88

Figure $4.10 \quad$ The geographic grid...................................... 90

Figure 4.11 Contributions of different data to regional geoid determination

Figure 5.1 Data area (in red the geoid area, in blue the gravity data and in green the DTM and DBM) 
Figure 5.2 Terrestrial gravity data distribution................................... 95

Figure 5.3 $\Delta$ g data source with 5' resolution.......................... 96

Figure 5.4 Digital Terrain and Bathymetric Model............................. 98

Figure 5.5 GPS/leveling distribution............................................. 99

Figure 5.6 IBGE GPS/leveling standard deviation............................ 100

Figure $6.1 \quad$ FFT scheme computation................................. 102

Figure 6.2 Helmert anomalies (white gaps there is no data)........... 103

Figure $6.3 \quad$ Geoid model by FFT .................................... 104

Figure $6.4 \quad$ LSC scheme computation................................ 105

Figure 6.5 Empirical covariance (blue) and fitted model (red) using EGM2008 up to $n=m$ 150............................... 106

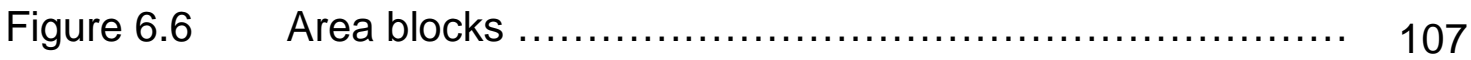

Figure $6.7 \quad$ Geoid model by LSC................................... 109

Figure $6.8 \quad$ Geoid heights residual.................................... 110

Figure 6.9 Difference between GPS/leveling and GEOIDSP ......... 112

Figure 6.10 Discrepancy GEOIDSP and GPS/leveling in the State of

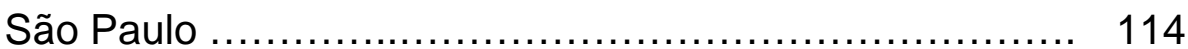

Figure 6.11 Histogram GPS/leveling and geoid models................ 116

Figure 6.12 GPS/leveling stations in the mountain area ................ 119

Figure 6.13 Relative comparison GPS/BM and GEOIDSP FFT

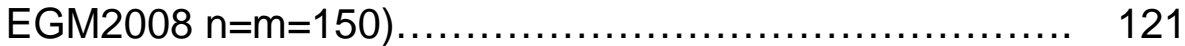

Figure 6.14 Relative comparison GPS/BM and GEOIDSP LSC

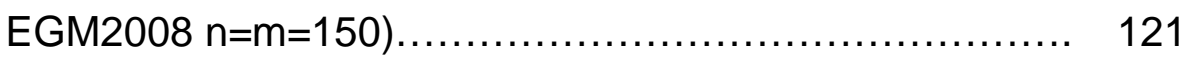

Figure 6.15 RMS of the geoid height (GPS/leveling) minus (GGMs) ... 128 
Figure 6.16 Differences between observed gravity disturbance minus models (left without RTC and right with RTC).............. 132 


\section{LIST OF TABLES}

Table 4.1 Main characteristics of the three satellite missions.......... 58

Table 4.2 Data periods of solutions and releases...................... 62

Table 5.1 GPS/leveling standard deviation....................... 97

Table 5.2 Statistics analysis of the geopotential models used in

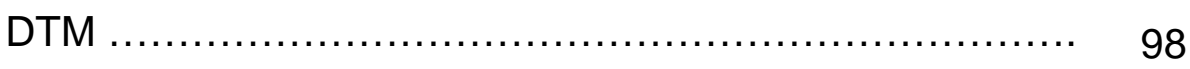

Table 6.1 Helmert anomalies and FFT geoid heights statistics........ 103

Table 6.2 Empirical and fitted variance values for the blocks........... 108

Table 6.3 LSC geoid heights statistics............................. 108

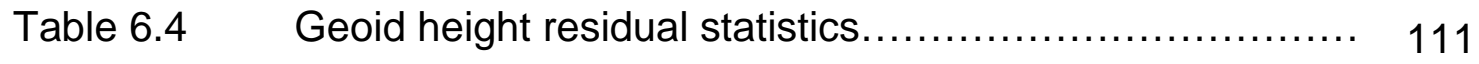

Table 6.5 Absolute comparison statistics (units in meters) ............. 118

Table 6.6 Absolute comparison statistics in the mountains (units in

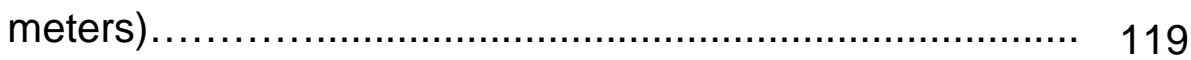

Table $6.7 \quad$ Relative comparison statistics............................ 122

Table 6.8 GGMs degree and order used in the comparisons.......... 123

Table 6.9 Statistics of the geoid height (GPS/leveling) minus

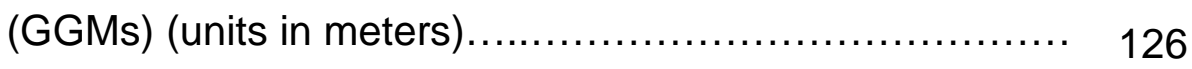

Table 6.10 Statistics of gravity disturbance differences (observed minus models) with RTC (units in mGal) .................. 130

Table 6.11 Statistics of gravity disturbance differences (observed minus models) without RTC (units in mGal) 


\section{LIST OF ABBREVIATIONS}

$B G I$

BM

BVP

CHAMP

DAE

DNSC08

DBM

DIR

DORIS

DOT

DTM2002

DTE

DTU

EGG

EGM96

EGM2008

ESA

EINGEN

EPUSP

FAPESP

FFT

GBVP
Bureau Gravimétrique International

Bench Mark

Boundary-Value Problem

CHAllenging Minisatellite Payload for Geophysical Research and Application

Direct Atmospheric Effect

Danish National Space Center 2008

Digital Bathymetric Model

Direct Solution

Doppler Orbit Determination and Radiopositioning Integrated on Satellite

Dynamic Ocean Topography

Digital Terrain Model 2002

Direct Topographical Effect

Danmarks Tekniske Universitet

Electrostatic Gravity Gradiometer

Earth Gravity Model 1996

Earth Gravity Model 2008

European Space Agency

European Improved Gravity Model of the Earth by New Techniques

Escola Politécnica da Universidade de São Paulo

Fundação de Amparo à Pesquisa do Estado de São Paulo

Fast Fourier Transform

Geodetic Boundary-Value Problem 


\begin{tabular}{|c|c|}
\hline GGM & Global Geopotential Model \\
\hline GLOBE & Global Land One-kilometer Base Elevation \\
\hline GNSS & Global Navigation Satellite System \\
\hline GOCE & Gravity field and steady-state Ocean Circulation Explorer \\
\hline GOCO & Gravity Observation COmbination \\
\hline GPS & Global Positioning System \\
\hline GRACE & Gravity Recovery And Climate Experiment \\
\hline GRAVSOFT & Geodetic Gravity Field Modelling Programs \\
\hline GSFC & Goddard Space Flight Center \\
\hline IAG & Instituto de Astronomia Geofísica e Ciências Atmosféricas \\
\hline IBGE & Fundação Instituto Brasileiro de Geografia e Estatística \\
\hline ICGEM & International Centre for Global Earth Model \\
\hline IGC & Instituto Geográfico e Cartográfico \\
\hline IGG & Instituto Geográfico e Geológico \\
\hline LSC & Least Squares Collocation \\
\hline LAGEOS & Laser Geodynamics Satellite \\
\hline MSL & Mean Sea Level \\
\hline NGA & National Geo-spatial Intelligence Agency \\
\hline NGDC & National Geophysical Data Center \\
\hline NOOA & National Oceanic and Atmospheric Administration \\
\hline ON & Observatório Nacional \\
\hline PGGM & Preconditioned Conjugate Gradient Method \\
\hline PETROBRAS & Petróleo do Brasil S.A. \\
\hline PITE & Primary Indirect Topographical Effect \\
\hline RTC & Residual Terrain Correction \\
\hline
\end{tabular}




$\begin{array}{ll}\text { RTM } & \text { Residual Terrain Model } \\ \text { SGG } & \text { Satellite Gravity Gradiometry } \\ \text { SHGEO } & \text { Stokes-Helmert GEOid software } \\ \text { SI } & \text { Système International d'unités } \\ \text { SITE } & \text { Secondary Indirect Topographical Effect } \\ \text { SPW } & \text { Space-Wise Solution } \\ \text { SSTI } & \text { Satellite-to-Satellite Tracking Instrument } \\ \text { SRTM } & \text { Shuttle Radar Topographic Mission } \\ \text { TC } & \text { Terrain Correction } \\ \text { TIM } & \text { Time-Wise Solution } \\ \text { WGS 84 } & \text { World Geodetic System 1984 }\end{array}$


- Dedication

- Acknowledgements

- Abstract

- Resumo

- List of Figures

- List of Tables

- List of Abbreviations

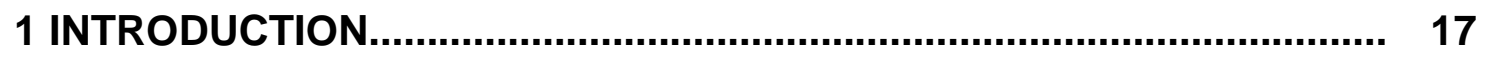

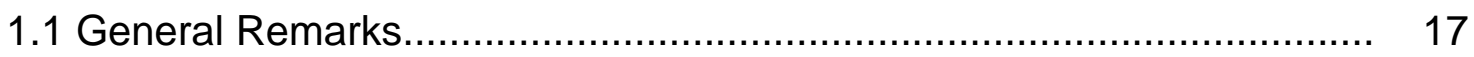

1.2 Thesis Objectives..................................................................... 19

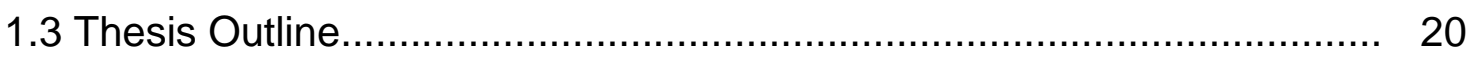

2 MATHEMATICAL FOUNDATIONS.................................................... 22

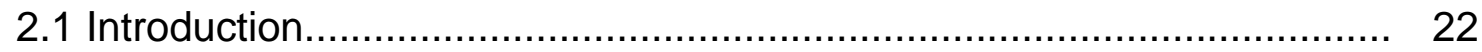

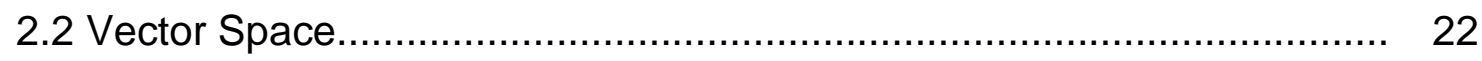

2.2.1 Linear space........................................................................ 22

2.2.2 Linear combination................................................................. 23

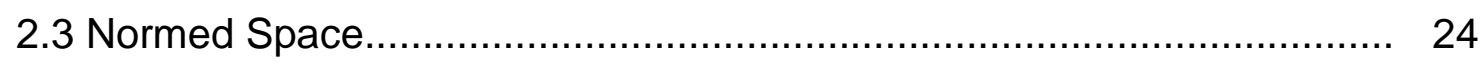

2.4 Inner Product Space................................................................. 25

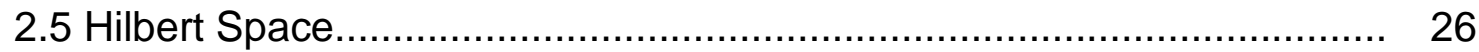

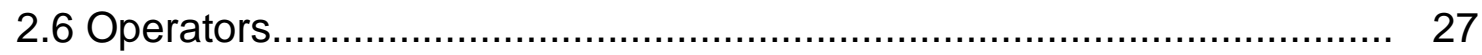

2.7 Least Squares Adjustment............................................................ 29

2.7.1 The least squares principle....................................................... 30

2.7.2 The alternatives of least squares................................................. 31

2.7.3 Linear and nonlinear models........................................................ 32

3 BASICS CONCEPTIONS...................................................................... 36

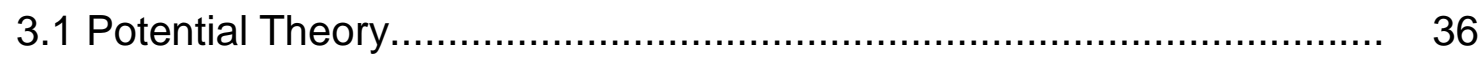

3.2 Gauss' and Green's Integral Formulas........................................... 40

3.3 Geodetic Boundary-Value Problem.................................................... 44 


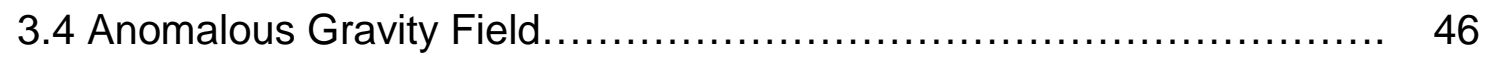

3.5 Spherical Harmonics Functions....................................... 48

3.6 The Geoid and Heights................................................ 52

4 GEOID ESTIMATION............................................................... 55

4.1 Global Gravitational Models............................................. 55

4.1.1 The new satellites missions....................................................... 57

4.1.1.1 The GOCE mission........................................................... 58

4.1.1.2 Global gravitational models based on GOCE................................. 62

4.1.1.3 The degree variance of the gravitational models............................. 64

4.2 Stokes' Integral......................................................... 67

4.2.1 Stokes' kernel modification............................................ 68

4.2.2 Fast Fourier Transform............................................. 73

4.3 Stokes-Helmert Scheme.............................................. 75

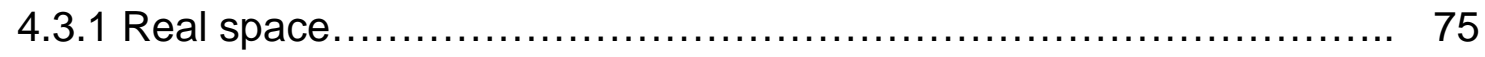

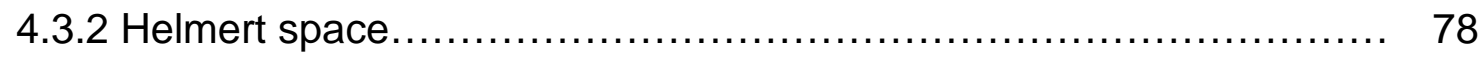

4.4 Least Squares Collocation......................................... 80

4.4 .1 Covariance function.................................................. 84

4.4 .2 Fast collocation........................................................ 86

4.5 The Spectral Decomposition (Remove-Restore) ........................ 92

5 DATA SET

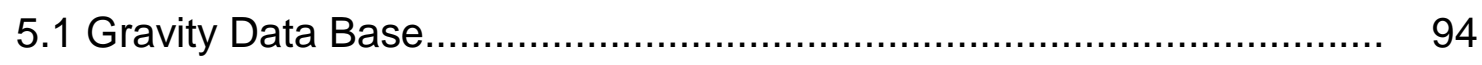

5.2 Geopotential Models.................................................................... 96

5.3 Digital Terrain Model..................................................................... 97

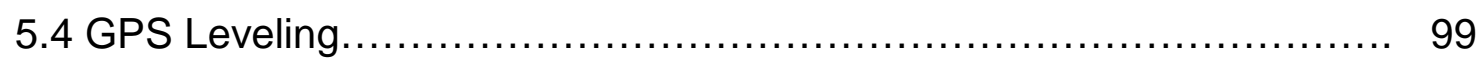

6 ESTIMATION PROCEDURES AND RESULTS................................... 101

6.1 Geoid Model Computed by FFT.................................................... 101

6.2 Geoid Model Computed by LSC....................................................... 104

6.3 Geoid Model Comparisons............................................... 109

6.3.1 Geoid height residual comparisons.................................. 109

6.3.2 Absolute comparisons............................................... 111 
6.3.2.1 Absolute comparisons in the mountains.................................... 118

6.3.3 Relative comparisons................................................ 120

6.4 GOCE Models Evaluation.............................................. 122

6.4.1 Comparison in terms of geoid heights............................... 122

6.4.2 Comparison in terms of gravity disturbances........................ 129

7 CONCLUSIONS AND RECOMMENDATIONS..................................... 134

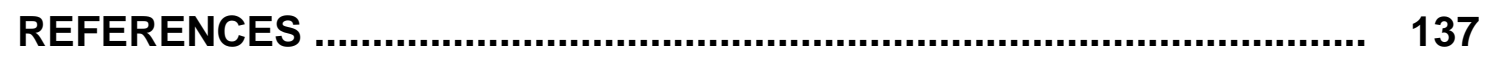




\section{INTRODUCTION}

\subsection{General Remarks}

Geodesy is concerned with the Earth's orientation parameters in the space, the shape and dimension of the Earth and estimation of its external gravity field. The shape computation of the Earth is carried out by the knowledge of the gravity field involving the mass distribution and the rotational effect of the planet. The determination of the potential function of the referred field should involve what is called "Geodetic Boundary-Value Problem". The reference surface of the Earth's gravity field is the geoidal surface, which is an equipotential surface. The geoid height represents the distance between the geoidal and the ellipsoidal surfaces along with the normal. The first surface has attracted some specific interests. Unlike the ellipsoidal surface, this one has a physical meaning.

The Boundary-Value Problem consists in the determination of the gravity field external to the masses where the boundary surface is unknown. Stokes proposed a formulation to obtain the disturbing potential as a function of the gravity anomaly on the geoidal surface. However, this proposition implies in some difficulties because it is a problem internal do the masses. A new formulation was proposed by Molodensky. It is a problem external to the masses which uses the physical surface as the boundary. In this sense, there is no need for the knowledge, even approximately, of a density distribution model within the crust between the geoidal and the physical surface. However, this surface has no physical meaning as the geoidal surface, because it is not an equipotential surface. The expression proposed by Molodensky is a nonlinear integral that cannot be solved directly. The solution is to linearize by introducing suitable approximate values. In this case, the real Earth is substituted by the normal Earth and the approximate solution for the boundary surface is the telluroid. What is computed is not the geoid, but the quasi-geoid. Gravity anomaly and vertical deflections are referred to the physical surface and not to the geoidal surface. Moreover, geoid heights are substituted by height anomalies 
and orthometric heights are replaced by normal heights. In this thesis the terminology geoid and geoid height will be used instead quasi-geoid and height anomaly.

In the geoid model computation, the gravity data provides the short wavelength, while the Global Geopotential Model (GGM) contributes in terms of medium and long wavelength components. The gravity data can be terrestrial, marine or airbone type and it is important that covers the interest area where the geoid model will be computed. A GGM is a set of coefficients of the Earth's gravitational potential function developed in series of spherical harmonic functions. It consists of a set of numerical values for certain parameters, the statistics of the errors associated with these values and a collection of mathematical expressions and algorithms that allows a user to perform synthesis and error propagation. In addition, the harmonic functions represented by a GGM should fulfill certain conditions due to the basic physics. It should represent a scalar function of position that is harmonic outside the attracting masses and vanishes at infinity as the reciprocal of the distance between attracted point and attracting mass elements. Nowadays, EGM2008 (Earth Gravity Model 2008) is the most used GGM in the geoid determination.

The spatial era contributed to the GGMs. The first model was published in the 1960s. The 1990s initialized the so called gravity decade. The modern gravitational missions were developed with specific objectives. The satellites were built to traffic in low orbit and they were designed with the most sophisticated equipments: GPS (Global Satellite System), DORIS (Doppler Orbit determination and Radiopositioning Integrated on Satellite) and laser system. From these missions three satellites were projected: CHAMP (CHAlleging Minisatellite Payload), GRACE (Gravity Recovery And Climate Experiment) and GOCE (Gravity field and steady-state Ocean Circulation Explorer). The last one was developed by the European Space Agency (ESA) and the main objective of the mission is to obtain a gravitational field model at the $~ 1-2 \mathrm{~cm}$ accuracy level for geoid height and at the 1-2 mGal level for gravity anomalies, and to achieve these results at a spatial resolution better than $100 \mathrm{~km}$. Since then, several geopotencial models of different degree and order have been published. In this thesis, GOCE-based models will be used, beyond EGM2008.

The geoid height can be computed using several techniques: numerical integration of Stokes' formula directly or using Fast Fourier Transform (FFT), Least Squares Collocation (LSC), spherical harmonic functions developed in series, direct 
calculation by the difference between the ellipsoidal height (from Global Navigation Satellite System - GNSS) and orthometric height (from spirit leveling), radar altimetry (in the oceans) and combination of them. In this thesis, Stokes' formula applying FFT and LSC were used to compute the geoid model. The first one, provides the disturbing potential as a function of the gravity anomalies on the geoid. The use of FFT is to speed up the process that involves a large quantity of data in a discrete form. The second one is a mathematical method in the determination of the anomalous gravity field combining geodetic measurements of different kinds. A modification in the original LSC also can be done to speed up the numerical procedures; this technique is called Fast Collocation.

Once the geoid model is computed, the most common way to analyze its quality is comparing over GPS observations on Bench Marks of spirit leveling network. The geoid height obtained from ellipsoidal height (GPS) minus normal height (spirit leveling) allows comparing with the geoid model height. Moreover, the comparison with recent GGMs is another option. In this case it is also possible to verify the quality of the geopotential model and the compatibility between them. The comparison involving two different methodologies can be done to ensure if they have the same behavior and to provide information of their consistency.

In 2008 an important project was initialized in the State of São Paulo. The project entitled Thematic Project (process: 06/04008-2) and supported by the State of São Paulo Research Foundation (FAPESP) has among its objectives, the gravity densification in the state and surrounding areas and the computation of the geoid model. Since then, some efforts were carried out to fill in the gravimetric gaps in this region providing an opportunity to perform a detailed study in the most developed region of the country.

\subsection{Thesis Objectives}

This research has two main objectives. The first one presents a detailed study and analysis of the geoid model in the State of São Paulo and surroundings, in Brazil using two different methodologies. The techniques are: Stokes' integration using Fast Fourier Transform and Least Squares Collocation applying Fast Collocation. 
Furthermore, a particular emphasis is given in terms of the positive points that one technique has in relation to the other one. Also, to verify the geoid models quality and consistency by comparisons over GPS observations on Bench Mark (BM) of spirit leveling network in a relative and absolute way.

The second objective is to provide a study on GOCE-based models in the same area. Since 2010 several global geopotential models using GOCE data have been published and have opened an opportunity to verify the quality of these models. In this case 13 models are tested and also EGM2008 using different degree and order to evaluate their potentiality. The models were compared in terms of geoid height and gravity disturbance.

\subsection{Thesis Outline}

This thesis is divided in 7 chapters. An outline of the essential structure of them is discussed below.

In chapter 2, the necessary background information regarding the mathematical foundations is presented. The discussion focuses on the functional analysis applied in Geodesy. Some concepts related to vector spaces, normed spaces and Hilbert spaces are discussed as well as the operators. Some examples useful in Geodesy are given. The end of the section addresses the principle of least squares adjustment and the related techniques.

In chapter 3, the basic concepts in Geodesy are discussed. The potential theory is described in detail coming up to Laplace's and Poisson's equations. The Geodetic Boundary-Value Problem is presented and the three boundary-value problems are commented. Gauss' and Green's integral formulas are explained, as well as the anomalous gravity field, where some quantities (disturbing potential, gravity anomaly, gravity disturbance) are mentioned. The section also presents the spherical harmonic functions and some concepts relate to height systems.

The next chapter focuses on the geoid estimation. Some features of the GGMs are explained. An overview of the new satellites missions is described. A special attention is given to GOCE mission. Its operation and the most important equipments 
are discussed, as well as, each GOCE-based model is mentioned in this section. Some characteristics regarding their solution are given. This chapter also presents a study related to the degree variance of the geopotencial models. Two plots show the degree variance and the error degree variance of seven geopotential models. The commission error was also computed for these seven models. Furthermore, the chapter describes Stokes' integral and some aspects of the kernel modification. Also, FFT technique is demonstrated. A comparison involving Stokes-Helmert scheme is discussed, which the real and the Helmert space are presented. A detailed description about Least Squares Collocation is performed including the covariance function details and the Fast Collocation technique. Finally, a brief discussion at the end of the chapter outlines the spectral decomposition.

In chapter 5, the data set used in the computation is described. Starting from the gravity data base, a description of the EGM2008, the digital terrain model used and finally, the GPS/leveling points are mentioned.

The geoid estimation procedures and the results are presented in chapter 6 . The schemes to compute the geoid model using FFT and LSC are described. The comparison involving the two methodologies is carried out, as well as the geoid models over GPS/leveling. Also, the results regarding the evaluation of the GOCEbased model in terms of geoid heights and gravity disturbances are presented.

Chapter 7 summarizes the main conclusions of this research and some considerations are proposed. 


\section{MATHEMATICAL FOUNDATIONS}

\subsection{Introduction}

In the late sixties and early seventies, Geodesy was introduced to functional analysis. Due to the work by Krarup, Moritz, Tengström, Hotine and others, some concepts related to vector, Hilbert and Banach spaces, and inverse problems were applied in geodetic problems. On the one hand, most of these problems are associated with three vector spaces: observations, parameters and mathematical model. On the other hand, Geodetic Boundary-Value Problem (GBVP) is a circular problem. In fact, this kind of problem is present in several sciences.

In the course of time, computers became available with a constant decrease in the price, and then geodesists attempted to solve some problems such as determining the shape of the Earth and describing the gravitational field, impossible without computers. Artificial satellites also contributed to the solution of these problems. This chapter outlines some definitions, properties and applications related to these settings.

\subsection{Vector Space}

\subsubsection{Linear space}

In mathematics, there is a list of the topics of mathematical situations involving linear problems. An example are the problems in the theory of linear integral equations e.g., problems related to Fourier and Laplace transforms.

According to Taylor (1958), a collection of elements, together with a certain structure of relations between elements or of rules of manipulation and combination, the whole supporting a mathematical development, is often called a space. A linear vector space $X$ over the field of real numbers is a set of elements $f, g, \ldots$, for which two 
types of operations are possible: addition of two elements and multiplication of an element of $X$ by a scalar. Vector addition has the following properties:

- $f+g=g+f$ (commutative);

- $\boldsymbol{f}+(\boldsymbol{g}+\boldsymbol{h})=(\boldsymbol{f}+\boldsymbol{g})+\boldsymbol{h}$ (associative);

- There is a unique element $0 \in X$ so that $0+f=f$ (neutral element);

- To each $\boldsymbol{f} \in \boldsymbol{X}$ there is a unique inverse $-\boldsymbol{f}$ so that $f+(-\boldsymbol{f})=0$ (inverse element).

Scalar multiplication has the properties as follows:

- $\alpha(f+g)=\alpha f+\alpha g, \alpha \in \Re$

- $\quad \alpha(\beta f)=(\alpha \beta) f$ for $\alpha, \beta \in \Re$ and $f \in X$ (associative);

- $(\alpha+\beta) f=\alpha f+\beta f$ (distributive);

- $1(f)=f \quad$ (neutral element).

\subsubsection{Linear combination}

The linear combination of vectors $f_{1}, f_{2}, f_{3}, \ldots, f_{n}$ over the vector space $X$ is a sum with the following form:

$$
\sum_{i=1}^{n} \alpha_{i} f_{i}=\alpha_{1} f_{1}+\ldots+\alpha_{n} f_{n}, \alpha_{i} \in \Re, f \in X
$$

As an example, let $\Omega$ be an open set in $\Re^{3}$ outside a sphere of radius $R$ and center at the origin and let $X$ consist of all the harmonic functions in $\Omega$ and regular at infinity (TSCHERNING, 1978). A finite dimensional subspace of this linear vector space is spanned by all the linear combinations of the solid spherical harmonics: 


$$
V_{i j}(P)=\left(\frac{R}{r}\right)^{i+1} P_{i j}(\sin \phi)\left\{\begin{array}{cc}
\cos j \lambda & 0 \leq j \leq i \\
\sin j \lambda & -j \leq i<0
\end{array}\right.
$$

where $r, \phi, \lambda$ are the distance from the origin, latitude and longitude of $P$, respectively. $P_{i j}$ are the normalized associated Legendre polynomials of degree $n$, cf. Heiskanen; Moritz (1967, eq. (1-77a,b)).

The harmonic functions in $\Omega$ and regular at infinity may be represented through a convergent series in solid spherical harmonics (TSCHERNING, 1978):

$$
f(P)=\sum_{i=0}^{\infty} \sum_{j=-i}^{i} \alpha_{i j} V_{i j}(P)
$$

Let $X$ be the space of harmonic functions considered in (2.2) and (2.3). In this case, gravity in a point $P$, with Cartesian coordinates $\left(x_{1}, x_{2}, x_{3}\right)$ is a non-linear function (TSCHERNING, 1978):

$$
g_{P}=\left(\sum_{i=1}^{3} \frac{\partial f}{\partial x_{i}}\right)^{\frac{1}{2}}
$$

while the gravity anomaly (cf. Heiskanen; Moritz (1967, (eq. 2-154))) is a well known quantity associated with a linear function, $\Delta_{g}$ :

$$
\Delta_{g}=-\frac{\partial f}{\partial r}-\frac{2}{r} f
$$

\subsection{Normed Space}

A normed space is a linear space with elements $f$ in which it is possible to define a real number designated $\|\boldsymbol{f}\|$ with the following properties: 
- $\|\boldsymbol{f}\| \geq 0$ (positivity);

- $\|\alpha f\|=|\alpha| \bullet \mid f \|$ (homogeneity);

- $\|\boldsymbol{f}+\boldsymbol{g}\| \leq\|f\|+\|\boldsymbol{g}\|$ (triangle inequality);

- $\|f\|=0$ if and only if $f=0$ (definiteness).

A normed space is also considered a metric space since to each pair of elements $\boldsymbol{f}, \boldsymbol{g}$ be possible to associate the distance $\boldsymbol{d}(\boldsymbol{f}, \boldsymbol{g})=\|\boldsymbol{f}-\boldsymbol{g}\|$ (TSCHERNING, 1978). A linear space which is a metric space in this way is called a normed linear space or a normed vector space. Regarding $f$ as a vector, its length is $\|f\|$; the length $\|f-g\|$ of the vector difference $\boldsymbol{f}-\boldsymbol{g}$ is the distance between the end points of the vectors $f$ and $g$ (TAYLOR, 1958).

\subsection{Inner Product Space}

The inner product space is a linear space if there is a mapping denoted by the inner product, with the following properties:

- $(f, g)=(g, f)$ (symmetry);

- $\left(f_{1}+f_{2}, g\right)=\left(f_{1}, g\right)+\left(f_{2}, g\right)$ (distributivity);

- $(\alpha f, g)=\alpha(\boldsymbol{f}, \boldsymbol{g})$ (homogeneity);

- $(f, f) \geq 0$ if and only if $f=0$ (positivity).

The expression "inner product space" and "pre-Hilbert space" are synonymous used by different authors (AUBIN, 1979), (ROBBINS, 2008).

A set of elements $\left(f_{i}, i=1,2, \ldots,\right)$ belonging to an inner product space is called orthogonal if

- $\left(f_{i}, f_{j}\right)=0$ for $i \neq j$ 
and orthonormal if also

- $\left(f_{i}, f_{j}\right)=1$

\subsection{Hilbert Space}

An inner product space which is infinite dimensional and complete is called a Hilbert space. $\operatorname{In} \Re^{n}$, a vector $\boldsymbol{x}$ may be represented in the form:

$$
\boldsymbol{x}=\alpha_{1} \boldsymbol{x}_{1}+\alpha_{2} \boldsymbol{x}_{2}+\ldots+\alpha_{n} \boldsymbol{x}_{n}
$$

where $\alpha_{1}, \alpha_{2}, \ldots, \alpha_{n}$ satisfy the orthonormality relations ( $\left.\S 2.4\right)$. Component $\boldsymbol{x}_{1}$ is the inner product of vector $x$ with base function $\alpha_{1}$

$$
x_{i}=\left(x, \alpha_{i}\right)
$$

This is the generalized Hilbert space $L_{2}$ (MORITZ, 1989). If it is assumed that there are base functions $\phi_{i}$ satisfying the following condition:

$$
\left(\phi_{i}, \phi_{j}\right)=\delta_{i j}
$$

for $i, j=1,2,3, \ldots$, and that given function $f$ may be expanded into a series of such base functions:

$$
f=\sum_{i=1}^{\infty} f_{i} \phi_{i}
$$

then the orthonormal system $\phi_{i}$ is called complete. According to Moritz (1989), a Banach space is a complete normed space, a complete inner-product space is an Euclidean space if its dimension is finite and a Hilbert space if its dimension is 
infinite. If all elements of the space can be represented as linear combinations of $n$ base elements, it is called finite dimension. For Hilbert space, the number of base elements is infinite cf. (2.9).

\subsection{Operators}

An operator is a mapping from one vector space $D$ (domain) to another vector space $R$ (range). Let $A$ and $B$ be two sets. The relation that associates $A$ and $B$, indicated by:

$$
f=T g
$$

where $f \in A$ and $g \in B$, are called operators so that

$$
T: D(T) \rightarrow R(T)
$$

in which $D(T)$ is the $T$ domain and $R(T)$ the range. If $D(T)=A$ and $R(T)=B$, so

$$
T: A \rightarrow B
$$

One operator $T$ is called linear operator since:

- the domain $D(T)$ and the range $R(T)$ are vector spaces associated with the same scalar field $K$;

- $\quad T(f+g)=T f+T g$ and $T(\alpha f)=\alpha T f \quad f, g \in D(T)$ and $\alpha \in K$.

For any $f_{1}, f_{2} \in D(T), x_{1} \neq x_{2} \Rightarrow T x_{1} \neq T x_{2}$ or equivalently: $T x_{1}=T x_{2} \Rightarrow x_{1}=x_{2}$. In these conditions, there is an inverse operator:

$$
T^{-1}: R(T) \rightarrow D(T)
$$


that associates each element $g \in R(T)$ to one element $x \in D(T)$.

Let $T: D(T) \rightarrow R(T)$ a linear operator, so

- $T^{-1}: R(T) \rightarrow D(T)$ if and only if $T x=0 \Rightarrow x=0$;

- if $T^{-1}$ exists, then it will be a linear operator;

- if $\operatorname{dim} D(T)=n<\infty$ and $T^{-1}$ exists, then $\operatorname{dim} R(T)=\operatorname{dim} D(T)$

Robbins (2008) presents an example to clarify the difference between a linear function and a linear operator. Let the two expressions (HEISKANEN; MORITZ, 1967):

$$
\begin{gathered}
N=\frac{R}{4 \pi G} \iint_{\sigma} \Delta g S(\psi) d \sigma \\
\left\{\begin{array}{l}
\xi \\
\eta
\end{array}\right\}=\frac{1}{4 \pi G} \iint_{\sigma} \Delta g \frac{d S}{d \psi}\left\{\begin{array}{l}
\cos \alpha \\
\sin \alpha
\end{array}\right\} d \sigma
\end{gathered}
$$

where $N$ is the geoid height, $G$ is a mean gravity value, $R$ is a mean radius of the Earth, and $\Delta g$ is the gravity anomaly. The $S(\psi)$ is the Stokes' function for a certain spherical distance $\psi$, between gravity anomaly contributing to the undulation or the deflection of the vertical (denoted by its components $\xi$ and $\eta$ ).

If $N$ or $\xi$ and $\eta$ are computed at one point only of fixed coordinates $(\varphi, \lambda)$, expressions (2.14) and (2.15) are considered. At a given point, the Stokes' function relates the vector space of gravity anomalies to the scalar values of $N$ or $\xi$ and $\eta$. However, considering the regional function of $N$, which is permissible since $N$ is a function of position $\varphi, \lambda$, then the integral in (2.14) acts as an operator. In this case, the Stokes' operator relates the vector of gravity anomalies to the regional position vector of undulations. The same holds true for the Vening Meinesz' case (ROBBINS, 2008). 


\subsection{Least Squares Adjustment}

In statistics, adjustment is a method of deriving estimates for stochastic variables and their distribution parameters from observed samples (MIKHAIL; AKERMANN, 1976). Least squares is by far the most common adjustment method. Gauss was the first to apply the least square adjustment to an astronomical problem, and since then, it has been introduced and used in a wide range of fields in science and engineering. Also, its practical importance was improved by the introduction of electronic computers and by connecting its concepts to statistics.

In the least square adjustment, a mathematical model must be specified by a certain number of variables (parameters or observations or both). There are three spaces involved in the adjustment problem:

- observations (measurements) space $\Re^{n}$;

- mathematical model space $\mathfrak{R}^{m}$;

- parameters space $\mathfrak{R}^{u}$.

Once a model is selected, a minimum number of distinct variables are defined. Denoting the minimum number of variables by $n_{0}$ and the total number of observations by $n$, the difference between them shows the redundancy denoted by $r$ and given by:

$$
r=n-n_{0}
$$

The redundancy corresponds to the degrees of freedom. When $n$ is larger then $n_{0}$, adjustment is needed in order to obtain a unique set of estimates for the model variables. 


\subsubsection{The least squares principle}

In a linear or nonlinear model or system, redundant and inconsistent, the solutions for the set of variables from each minimum subset would yield a different result. No single result is possible from redundant observations unless an additional criterion is introduced. In this situation the least squares application to derive a single set of estimates for all the model variables, with certain optimum properties is used (MIKHAIL; AKERMANN, 1976).

The original set of observations, which will be denoted by vector $L_{b}$, includes redundant observations and is inconsistent with the model, is replaced in the adjustment by another set of estimates $L_{a}$ which satisfies the model.

The set of estimates $L_{a}$ is different from the original set $L_{b}$ and the difference between them is given by:

$$
V=L_{a}-L_{b}
$$

where $V$ is known as corrections or residuals vectors.

Vector $L_{a}$, which satisfies the model, depends on the choice of vector $V$. According to Gemael (1994), any criterion should be established to obtain a single solution to $L_{a}$. The option proposed by Gauss and Legendre is to accept as best estimates of $L_{a}$, the value that makes the sum of the residual squares minimum.

The least squares principles states that:

$$
\phi=V^{T} P V \rightarrow \text { minimum }
$$

where $V$ is the residual vector and $P$ is the weight matrix of the observations given by:

$$
P=\sigma_{0}^{2} \Sigma_{L_{b}}^{-1}
$$


where $\sigma_{0}^{2}$ is the a priori variance factor and $\Sigma_{L_{b}}$ is the variance-covariance matrix of the error of the observations. It is worth mentioning that the least squares principle does not require a priori knowledge of the distribution associated with the observations.

\subsubsection{The alternatives of least squares}

The mathematical model is the first point of the adjustment task. Once the model is established, the practical and computational aspects are employed to select a particular technique of least squares. Figure 2.1 shows the relationship between these issues.

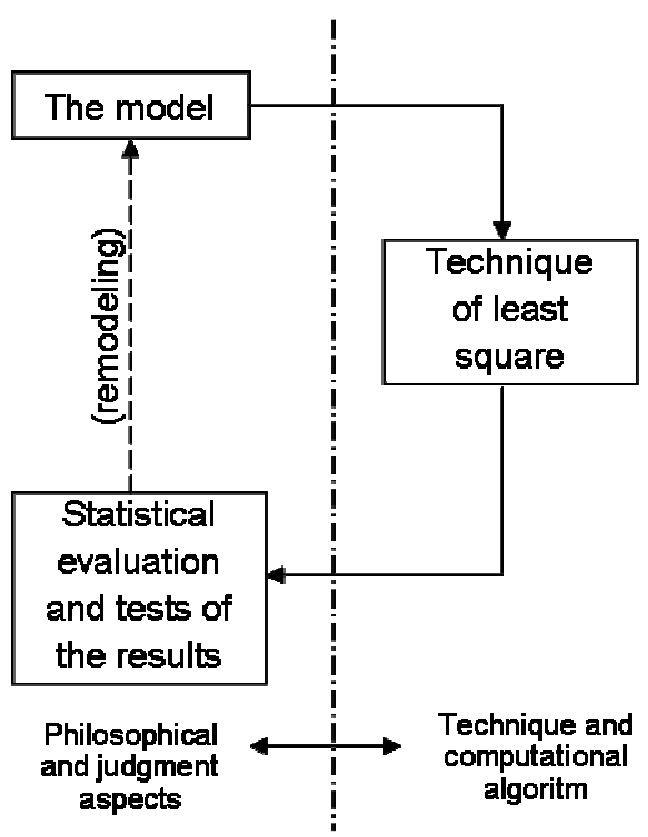

Figure 2.1 - Relationship between the adjustments issues (Mikhail; Akermann, 1976).

The least squares computations generate updated estimates of all model variables as well as their respective covariance matrix. After the computational algorithm is applied, the next step is the statistic results evaluation. It is a judgment operation of the model and the observations.

Depending on the variables (observations and/or parameters) involved in the model, the adjustment can be solved using one of the follows alternatives: 
- The mathematical model involves only the observation vector $\left(L_{a}\right)$. In this case, parameters do not participate in the adjustment. The model is represented by:

$$
F\left(L_{a}\right)=0
$$

- The observations $\left(L_{a}\right)$ and parameters $\left(X_{a}\right)$ are involved in the mathematical model, and the model is expressed by:

$$
F\left(L_{a}, X_{a}\right)=0
$$

- The model also involves observations $\left(L_{a}\right)$ and parameters $\left(X_{a}\right)$; however, the observations are explicit functions of the parameters:

$$
L_{a}=F\left(X_{a}\right)
$$

\subsubsection{Linear and nonlinear models}

In general, equations involved in an adjustment problem can be linear or nonlinear. However, least squares computations are usually carried out with linear functions, once it is rather difficult and impractical to obtain the solution using nonlinear functions. When the equations in the model are originally nonlinear, series expansions and/or Taylor's series are often used as linearization. In this case, only the zero and first-order terms are used and all the other higher-order terms are neglected.

The linearization basis of a function is the Taylor's series expansion. This series provides a function value in the form:

$$
y=f(x) .
$$

At point $x$, when the function value $x=x_{0}$ is known, the series can be written: 


$$
f(x)=f\left(x_{0}\right)+f^{\prime}\left(x_{0}\right) \frac{\left(x-x_{0}\right)}{1 !}+f^{\prime \prime}\left(x_{0}\right) \frac{\left(x-x_{0}\right)^{2}}{2 !}+\ldots
$$

The equal power or higher than the second can be neglected for $x$ values close to $x_{0}$. Nearby $x_{0}$ the curve $f(x)$ can be replaced by a line

$$
f(x)=f\left(x_{0}\right)+f^{\prime}\left(x_{0}\right) \Delta x .
$$

Expression (2.25) represents the linear model for one variable, where $\Delta x=\left(x-x_{0}\right)$ and $f\left(x_{0}\right)$ is the angular coefficient of the line at point $x_{0}$. Figure 2.2 shows the linearization process.

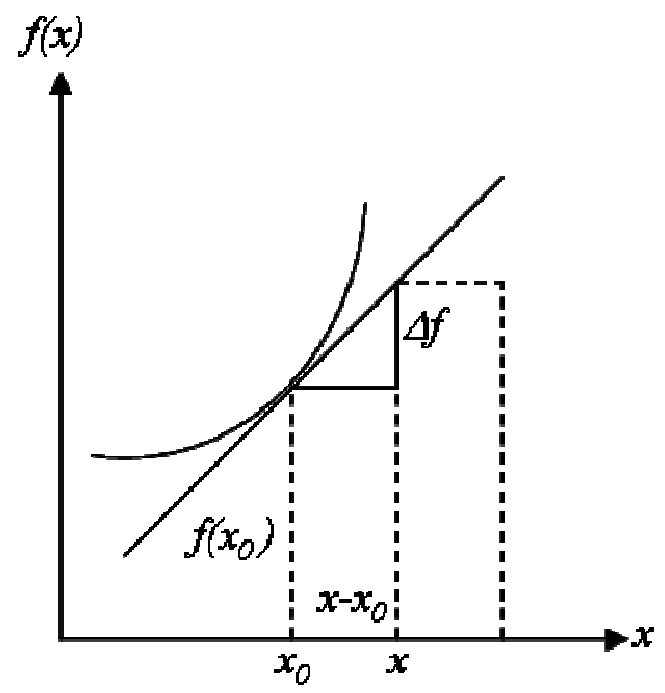

Figure 2.2 - Original and linearized function.

Considering a two-variable function, the linear approach is as follows:

$$
f(X)=f\left(x_{1}, x_{2}\right)
$$

and

$$
f\left(x_{1}, x_{2}\right)=f\left(x_{1}^{0}, x_{2}^{0}\right)+\left.\frac{\partial f}{\partial x_{1}}\right|_{X^{0}}\left(x_{1}-x_{1}^{0}\right)+\left.\frac{\partial f}{\partial x_{2}}\right|_{X^{0}}\left(x_{2}-x_{2}^{0}\right) .
$$


Applying

$$
X=\left[\begin{array}{l}
x_{1} \\
x_{2}
\end{array}\right] ; \quad \Delta x=\left[\begin{array}{l}
x_{1}-x_{1}^{0} \\
x_{2}-x_{2}^{0}
\end{array}\right] ; \quad X^{0}=\left[\begin{array}{l}
x_{1}^{0} \\
x_{2}^{0}
\end{array}\right] ; \quad \frac{\partial f}{\partial X}=\left[\frac{\partial f}{\partial x_{1}} \frac{\partial f}{\partial x_{2}}\right]
$$

the result is

$$
f(X)=f\left(X^{0}\right)+\left.\frac{\partial f}{\partial X}\right|_{X^{0}} \Delta X
$$

The linear approach considering $n$ variables $x_{1}, x_{2}, \ldots ., x_{n}$ and $m$ functions $f_{1}, f_{2}, \ldots, f_{m}$ is

$$
\begin{aligned}
& f_{1}(X)=f_{1}\left(X^{0}\right)+\left.\frac{\partial f_{1}}{\partial X}\right|_{X^{0}} \Delta X \\
& f_{2}(X)=f_{2}\left(X^{0}\right)+\left.\frac{\partial f_{2}}{\partial X}\right|_{X^{0}} \Delta X \\
& \ldots \ldots \ldots \ldots \ldots \ldots \ldots \ldots \ldots \ldots \ldots \ldots \ldots \ldots \ldots \ldots \ldots \ldots \\
& f_{m}(X)=f_{m}\left(X^{0}\right)+\left.\frac{\partial f_{m}}{\partial X}\right|_{X^{0}} \Delta X
\end{aligned}
$$

and applying

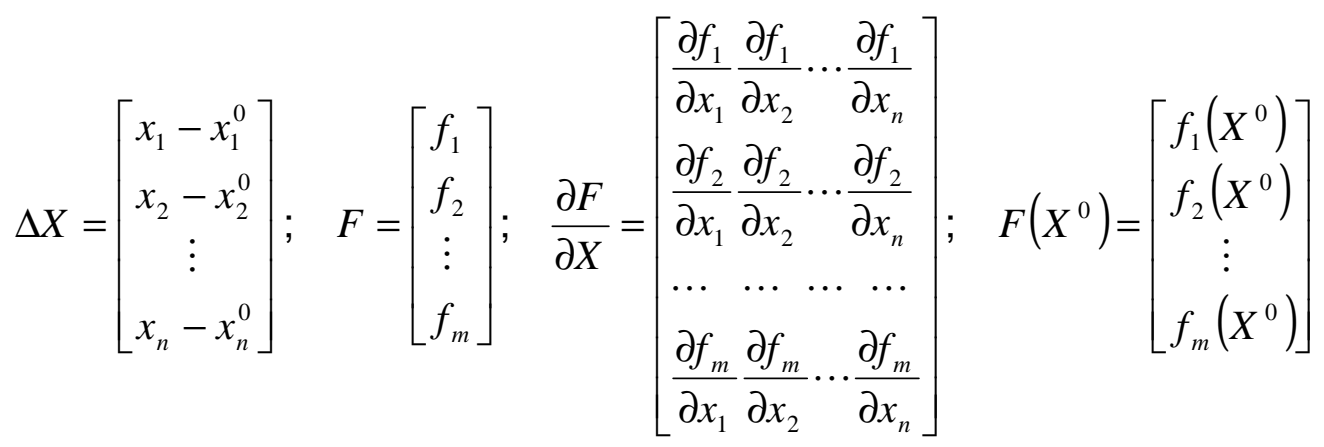

the Taylor's series linear approach in a matrix form is given by: 


$$
F(X)=F\left(X^{0}\right)+\left.\frac{\partial F}{\partial X}\right|_{X^{0}} \Delta X
$$




\section{BASICS CONCEPTIONS}

\subsection{Potential Theory}

The physical attraction that a body exerts on near objects is the result of the gravitational force. Isaac Newton formulated the law of universal gravitation in his work Philosophiae Naturalis Principia Mathematica, published in 1687, which also describes Newton's laws - laws of motion of celestial bodies, the basis of classical mechanics.

According to Newton's law of gravitation, a point with mass $m_{1}$ in the universe attracts another point with mass $m_{2}$ with a force that is directly proportional to the product of their masses and inversely proportional to the square of the distance between them (3.1) (HOFMANN-WELLENHOF; MORITZ, 2006):

$$
F=G \frac{m_{1} m_{2}}{l^{2}}
$$

where $G$ is known as "gravitation universal constant", which in SI units (Système International d'unités) has the value :

$$
G=6,6742 * 10^{-11} \mathrm{~m}^{3} \mathrm{~kg}^{-1} \mathrm{~s}^{-2}
$$

To avoid possible ambiguity, it is convenient to consider one of the particles as "attracting mass" and the other as "attracted mass". For simplicity, if the attracted mass is considered equal to unity and the attracting mass denoted by $m$, the formula reads as follow:

$$
F=G \frac{m}{l^{2}}
$$


In terms of a coordinate system $x, y, z$ the force may be represented by a vector $F$. The coordinates of the attracting mass $m$ are indicated by $\xi, \eta, \zeta$ and the coordinates of the attracted point $P$ by $x, y, z$. The force may be represented by a vector $F$ with magnitude $F$. The components of $F$ are given by:

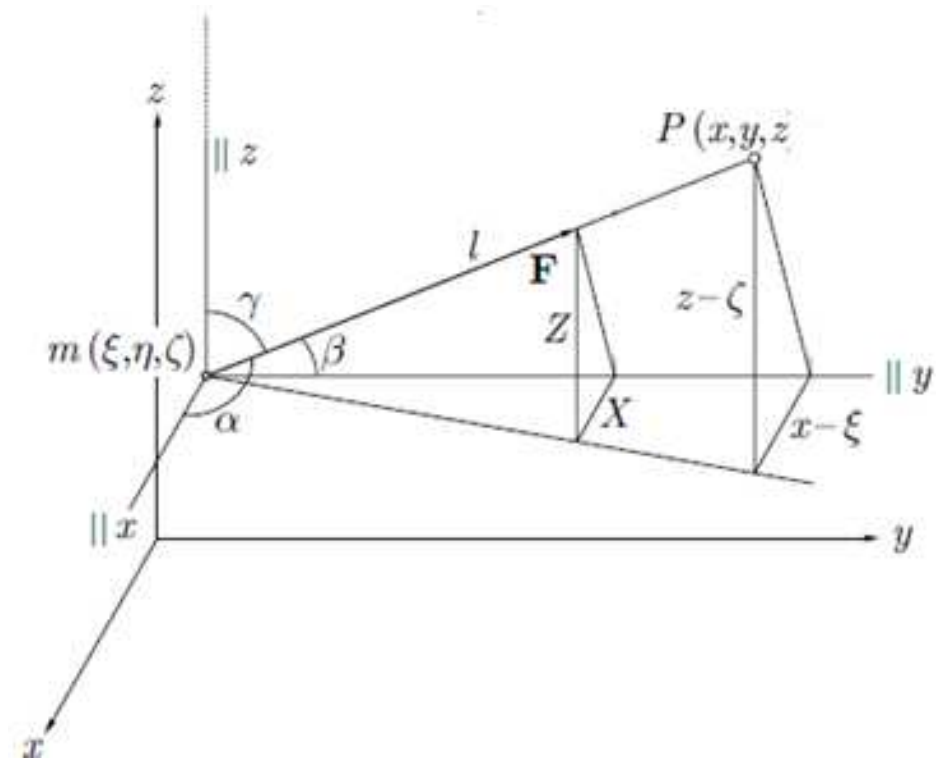

Figure 3.1 - The components of the gravitational force (Hofmann-Wellenhof; Moritz, 2006).

$$
\begin{aligned}
& X=-F \cos \alpha=-\frac{G m}{l^{2}} \frac{x-\xi}{l}=-G m \frac{x-\xi}{l^{3}}, \\
& Y=-F \cos \beta=-\frac{G m}{l^{2}} \frac{y-\eta}{l}=-G m \frac{y-\eta}{l^{3}}, \\
& Z=-F \cos \gamma=-\frac{G m}{l^{2}} \frac{z-\zeta}{l}=-G m \frac{z-\zeta}{l^{3}},
\end{aligned}
$$

where

$$
l=\sqrt{(x-\xi)^{2}+(y-\eta)^{2}+(z-\zeta)^{2}}
$$

Components $X, Y, Z$ of $F$ can be expressed by: 


$$
\begin{aligned}
& X=\frac{\partial V}{\partial x} \\
& Y=\frac{\partial V}{\partial y} \\
& Z=\frac{\partial V}{\partial z}
\end{aligned}
$$

In vector notation, expression (3.5) is written as:

$$
F=[X, Y, Z]=\operatorname{grad} V
$$

The potential of gravitation or Newtonian is a scalar function defined by:

$$
V=G \frac{m}{l}
$$

and for a system of several point masses, the potential of the system is the sum of the individual contributions (3.7):

$$
V=\frac{G m_{1}}{l_{1}}+\frac{G m_{2}}{l_{2}}+\ldots+\frac{G m_{n}}{l_{n}}=G \sum_{i=1}^{n} \frac{m_{i}}{l_{i}}
$$

Assuming that point masses are distributed continuously over a volume $v$, the sum of (3.8) becomes an integral and the gravitational potential $V$ may be expressed by the formula:

$$
V(P)=V(x, y, z)=G \iiint_{v} \frac{\rho(Q)}{l} d v_{Q}
$$

where $P$ is a point having coordinates $(x, y, z), Q$ is a point variable within the Earth's body, $l$ is the distance between $P$ and $Q$, and $\rho(Q)$ is the mass density at $Q$.

The expression (3.9) has only theoretical value because its practical use would require the knowledge of the detailed density distribution within the Earth, which is 
not possible (MORITZ, 1989). Let us introduce another important quantity in Physical Geodesy, the potential of the centrifugal force:

$$
\Phi=\frac{1}{2} \omega^{2}\left(x^{2}+y^{2}\right)
$$

where $\omega$ is the angular velocity of the Earth's rotation.

The sum of (3.9) and (3.10) results in the gravity potential $W$ :

$$
W=\Phi+V
$$

The field of potential $V$ is called the gravitational field and the field of potential $W$ is the gravity field. Thus, gravitational vector $\vec{f}$ is the gradient of $V$ :

$$
\vec{f}=\operatorname{grad} V=\left(\begin{array}{c}
V_{x} \\
V_{y} \\
V_{z}
\end{array}\right)
$$

and vector gradient $\vec{g}$ is the gradient of $W$ :

$$
\vec{g}=\operatorname{grad} W=\left(\begin{array}{l}
W_{x} \\
W_{y} \\
W_{z}
\end{array}\right) .
$$

It is worth mentioning that the gravitational gradient tensor $V_{i j}$ :

$$
V_{i j}=\left[\begin{array}{lll}
V_{x x} & V_{x y} & V_{x z} \\
V_{y x} & V_{y y} & V_{y x} \\
V_{z x} & V_{z y} & V_{z z}
\end{array}\right]
$$

where the elements are the second-order partial derivatives of $V$. Similarly, the second-order derivatives of $W$ form the gravity gradient tensor. 
The trace of the matrix in (3.14) is the Laplacian of $V$ :

$$
\Delta V=V_{x x}+V_{y y}+V_{z z} .
$$

Outside the attracting masses, $V$ satisfies Laplace's equation:

$$
\Delta V=0
$$

and inside the masses it satisfies Poisson's equation:

$$
\Delta V=-4 \pi G \rho .
$$

Equations (3.16) and (3.17) are very important in Geodesy. The first one is used in the solution of the Geodetic Boundary-Value Problem (GBVP) outside the attracting masses. The second one offers the possibility to solve the GBVP inside the masses, provided the density distribution model be known.

\subsection{Gauss' and Green's Integral Formulas}

Green's theorems are the basic formulation of potential theory and they are important tools for certain problems of theoretical Geodesy. Starting with Gauss' and Ostrogradski's integral formula (HEISKANEN; MORITZ, 1967):

$$
\iiint_{v} d i v F d v=\iint_{S} F_{n} d S
$$

where $v$ is the volume enclosed by the surface $S, F_{n}$ is the projection of vector $F$ along normal $n$ of the surface oriented outside the surface and $\operatorname{div} F$ is the so-called divergence of vector $F$.

The right-hand side of (3.18) represents the flux of force, in geodetic case gravitational flux, also in analogy to fluid flow. Gauss's formula may be expressed in terms of function $V$, for any force whose components can be derived from a potential 
$V$ according to (3.7). Inserting $F_{n}=\frac{\partial V}{\partial n}$ and the relation $F=\Delta V$ into (3.18), Gauss' integral formula for the potential can be expressed by:

$$
\iiint_{v} \Delta V d v=\iint_{S} \frac{\partial V}{\partial n} d S
$$

From (3.19) is possible to derive the following formulas:

$$
\begin{aligned}
& X=U \frac{\partial V}{\partial x} \\
& Y=U \frac{\partial V}{\partial y} \\
& Z=U \frac{\partial V}{\partial z}
\end{aligned}
$$

where $U, V$ are functions of $x, y, z$. The normal component of vector $F=(X, Y, Z)$ is then given by:

$$
F_{n}=U \frac{\partial V}{\partial n}
$$

and the divergence of the vector $F$ is:

$$
\operatorname{div} F=\frac{\partial U}{\partial x} \frac{\partial V}{\partial x}+\frac{\partial U}{\partial y} \frac{\partial V}{\partial y}+\frac{\partial U}{\partial z} \frac{\partial V}{\partial z}+U \Delta V
$$

Applying Gauss' divergence theorem in (3.18), one obtain the Green's first identity:

$$
\iiint_{v} U \Delta V d v+\iiint_{v}\left(\frac{\partial U}{\partial x} \frac{\partial V}{\partial x}+\frac{\partial U}{\partial y} \frac{\partial V}{\partial y}+\frac{\partial U}{\partial z} \frac{\partial V}{\partial z}\right) d v=\iint_{S} U \frac{\partial V}{\partial n} d S
$$

Interchanging $U$ and $V$ and subtracting the new equation from the original, the Green's second identity reads as follows: 


$$
\iiint_{v}(U \Delta V-V \Delta U) d v=\iiint_{S}\left(U \frac{\partial V}{\partial n}-V \frac{\partial U}{\partial n}\right) d S .
$$

Attention should be given to the case in which $U=\frac{1}{l}$, where $l$ is the distance from a certain fixed point $P$. If $P$ is outside surface $S$, then $1 / l$ is regular inside and on $S$, and $U$ satisfies the condition mentioned above. On the other hand, if $P$ is inside or on $S$, then $1 / l$ becomes infinite at a point in $v$ and (3.24) cannot be applied directly and must be modified. Omitting the derivation, maintaining $n$ as the outer normal and reversing $\partial / \partial n$ Green's third identity for the exterior of surface $S$ is derived:

$$
\iiint_{v} \frac{1}{l} \Delta V d v=-p V-\iint_{S}\left[\frac{1}{l} \frac{\partial V}{\partial n}-V \frac{\partial}{\partial n}\left(\frac{1}{l}\right)\right] d S
$$

where

$$
p= \begin{cases}4 \pi & \text { if } P \text { outside } S \\ 2 \pi & \text { if } P \text { on } S \\ 0 & \text { if } P \text { inside } S\end{cases}
$$

This is Green's third identity for the exterior of surface $S$. It is valid for functions $V$ that, besides satisfying the general requirements for Green's identities, satisfies certain conditions at infinity, such as vanishing there (HEISKANEN; MORITZ, 1967). Considering the Earth and gravitational potential $W$ and applying the third identity (3.25) to a point on the Earth's surface and $p=2 \pi$ :

$$
\iiint_{v}^{1} \frac{1}{l} \Delta W d v+2 \pi W-\iint_{S}\left[\frac{1}{l} \frac{\partial W}{\partial n}-W \frac{\partial}{\partial n}\left(\frac{1}{l}\right)\right] d S=0 .
$$

Function $W$ satisfies expression (3.11) so that:

$$
\Delta W=-4 \pi G \rho+2 \omega^{2}
$$


which is similar to Poisson's expression (3.17) and $\omega$ is the angular velocity of the Earth's rotation. Let $v$ be the Earth and $S$ its physical surface. Then

$$
\frac{\partial W}{\partial n}=-g_{n}
$$

which is the component of gravity normal to the Earth's surface $S$. Substituting (3.25) into (3.26) and (3.27):

$$
\iiint_{v}^{1} \frac{1}{l}\left(-4 \pi G \rho+2 \omega^{2}\right) d v+2 \pi W+\iint_{S}\left[W \frac{\partial}{\partial n}\left(\frac{1}{l}\right)+\frac{g_{n}}{l}\right] d S=0
$$

and according to (3.9)

$$
W=G \iiint_{v} \frac{\rho}{l} d v+\frac{1}{2} \omega^{2}\left(x^{2}+y^{2}\right)
$$

it finally has:

$$
-2 \pi W+\iint_{S}\left[W \frac{\partial}{\partial n}\left(\frac{1}{l}\right)-\frac{1}{l} \frac{\partial W}{\partial n}\right] d S+2 \pi \omega^{2}\left(x^{2}+y^{2}\right)+2 \omega^{2} \iiint_{v} \frac{d v}{l^{\prime}}=0
$$

that relates surface $S$ to gravity potential $W$ and gravity $g$. If $W$ and $g$ are given, it is reasonable to assume that one can somehow solve the above equation for surface $S$. Actually, this equation may be considered the mathematical basis for determining the physical surface $S$ of the Earth from the potential $W$ and gravity $g$, or in other words, of the Boundary-Value Problem of Physical Geodesy according to Molodensky.

Expression (3.30) is a nonlinear integral that cannot be solved directly. The solution is to linearize by introducing suitable approximate values. In this case, the real Earth is substituted by the normal Earth; the actual potential $W$ is thus approximated by a normal potential $U$ and the approximate solution for $S$ is telluroid $\Sigma$. Details about the linearization can be found in (HEISKANEN; MORITZ, 1967). After the linearization process, the following expression is derived: 


$$
T-\frac{1}{2 \pi} \iint_{\Sigma}\left[\frac{\partial}{\partial n}\left(\frac{1}{l}\right)-\frac{1}{\gamma} \frac{\partial \gamma}{\partial h} \frac{\cos \beta}{l}\right] T d \Sigma=\frac{1}{2 \pi} \iint_{\Sigma} \frac{1}{l}\left[\Delta g-\gamma\left(\xi \tan \beta_{1}+\eta \tan \beta_{2}\right)\right] \cos \beta d \Sigma
$$

This is a linear integral expression of the second kind for disturbing potential $T$ or for height anomaly $\zeta=T / \gamma$.

\subsection{Geodetic Boundary-Value Problem}

The knowledge of the values of a given variable on the surface and thereafter, the determination of a function that relates to that variable, leading to what is called "Boundary-Value Problem" (BVP) (BLITZKOW, 1996). Briefly, the aim of the BVP is to establish a function for a given body. The determination of the external gravity field of the Earth from terrestrial observational data is related to the formulation of BVP in Geodesy with respect to the Laplace-Poisson differential equation (3.16) and (3.17).

As said before, the Geodetic Boundary-Value Problem is to determine the gravity field outside the masses whose surface is unknown. Stokes proposed a formulation to obtain the disturbing potential as a function of gravity anomalies on the geoidal surface. However, this proposition implies some difficulties because it is a problem inside the masses. In this sense, a new formulation was proposed by Molodensky. This is an external problem to the masses that uses the physical surface as a boundary. In this way it is not necessary to know, even approximately, the density distribution model inside the crust. However, this surface does not have the same physical meaning than the geoidal surface, because it is not an equipotential surface.

The potential theory presents three boundary-value problems. To be simple it is assumed that the disturbing potential $T$ (see 3.37) is given on a sphere of radius $R$. So it shall treat simply the problems:

- Dirichlet: the knowledge of the values of function $T$ on surface $S$ allows determining function $T$ in such a way that it is harmonic either inside or outside $S$, such that: 


$$
\begin{cases}\Delta T=0 & r>R \\ T=\bar{T} & r=R\end{cases}
$$

- Neumann: under the same conditions above, the normal derivative of function $T$ on surface $S, \partial T / \partial r$, is known for the determination of $T$, harmonic either inside or outside $S$ :

$$
\begin{cases}\Delta T=0 & r>R \\ \frac{\partial T}{\partial r}=-\delta_{g} & r=R\end{cases}
$$

- Hilbert: from the values of a linear combination of function $T$ and of its normal derivative on surface $S$, function $T$ is determined in the previous conditions:

$$
\begin{cases}\Delta T=0 & r>R \\ \frac{\partial T}{\partial r}+\frac{2}{r} T=-\Delta g & r=R\end{cases}
$$

These problems do not present a single solution, because infinite mass distributions may generate the same potential. The third problem is relevant to Geodesy, since the determination of the disturbing potential somehow relates to it. Gravity anomaly is represented as a linear combination between the disturbing potential and its normal derivative, and it is expressed by a differential equation known as the "fundamental equation of Physical Geodesy". 


\subsection{Anomalous Gravity Field}

An ellipsoid of revolution, generated by rotating an ellipse around the minor axis, having the same angular velocity and the same mass as the real Earth, whose potential $U_{0}$ constant on the surface, is equal to the potential on geoidal surface $W_{0}$, with its center at the geocenter, is called normal Earth. The normal gravity potential (3.35) and the normal gravity vector (3.36) may be defined as:

$$
U=\Phi+U_{g}
$$

where $U_{g}$ is the normal gravitational potential

$$
\vec{\gamma}=\operatorname{grad} U=\left(\begin{array}{l}
U_{x} \\
U_{y} \\
U_{z}
\end{array}\right)
$$

Normal gravity potential $U$ is a good approximation for gravity potential $W$, and the difference is called anomalous or disturbing potential $T$.

$$
T=W-U
$$

Introducing some substantial quantities (gravity anomaly and gravity disturbance), which Figure 3.2 will help in understanding. The figure shows Earth's gravity on the geoid surface and the normal gravity on the ellipsoid surface. 


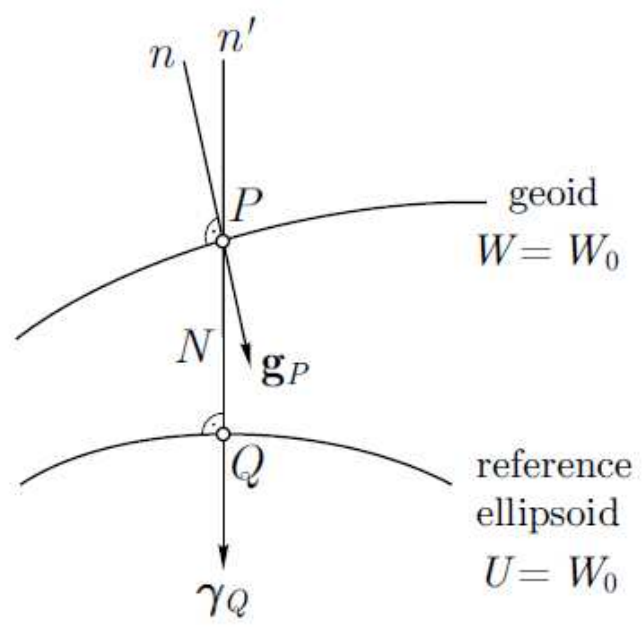

Figure 3.2 - Geoid and ellipsoid surfaces (Hofmann-Wellenhof; Moritz, 2006).

Considering the Earth's gravity on the geoid $\left(g_{P}\right)$ and the normal gravity on the surface of the reference ellipsoid $\left(\gamma_{Q}\right)$, the difference in magnitude is called gravity anomaly $(\Delta g)$ :

$$
\Delta g=g_{P}-\gamma_{Q} .
$$

The difference in direction is the deflection of the vertical and it has two components: a north-south component $\xi$ and an east-west component $\eta$. Expression (3.38) reflects the gravity anomaly in terms of difference in magnitude.

Another important quantity is the difference in the same point between observed gravity $\left(g_{P}\right)$ and normal gravity $\left(\gamma_{P}\right)$, known as the gravity disturbance $(\delta g)$.

$$
\delta g=g_{P}-\gamma_{P}
$$

By Brun's theorem is possible to relate the geoid height to the disturbing potential:

$$
N=\frac{T}{\gamma}
$$

From (3.40) and relating different quantities of the anomalous gravity field, it is possible to establish equivalent relations, and one of them has been called the 
fundamental equation of Physical Geodesy (HOFMANN-WELLENHOF; MORITZ, 2006):

$$
\Delta g=-\frac{\partial T}{\partial h}+\frac{1}{\gamma} \frac{\partial \gamma}{\partial h} T
$$

This formula relates the measured quantity $\Delta g$ to the unknown disturbing potential $T$. Some advantage exists when the sphere is considered an approximation of the Earth's shape instead of a reference ellipsoid. The differences are very small, and in terms of flattening. In several problems, it is an acceptable approximation. Then, this boundary condition takes the form (MORITZ, 1989):

$$
\Delta g=-\frac{\partial T}{\partial r}-\frac{2}{R} T
$$

where $R$ is the mean radius of the Earth. Expression (3.42) expresses one condition for the third Boundary-Value Problem solution (3.34).

\subsection{Spherical Harmonics Functions}

The spherical harmonics are the most important harmonic functions. Laplace's equation in spherical coordinates $r$ (radius vector), $\theta$ (polar distance), $\lambda$ (geocentric longitude) is represented in Figure 3.3.

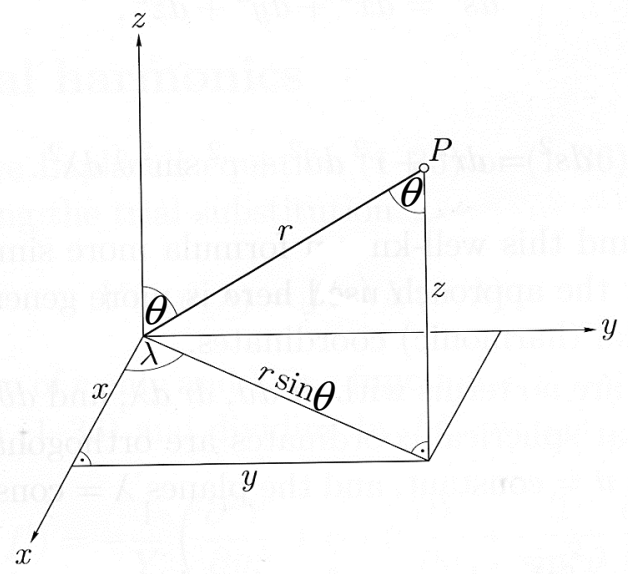

Figure 3.3 - Spherical and rectangular coordinates (Hofmann-Wellenhof; Moritz, 2006). 
Spherical coordinates are related to rectangular coordinates $x, y, z$ by expressions (HOFMANN-WELLENHOF; MORITZ, 2006):

$$
\begin{aligned}
& x=r \sin \theta \cos \lambda \\
& y=r \sin \theta \sin \lambda \\
& z=r \cos \theta .
\end{aligned}
$$

Determining the element of arc (element of distance) $d s$ in these coordinates

$$
\begin{aligned}
& d x=\frac{\partial x}{\partial r} d r+\frac{\partial x}{\partial \theta} d \theta+\frac{\partial x}{\partial \lambda} \partial \lambda \\
& d y=\frac{\partial y}{\partial r} d r+\frac{\partial y}{\partial \theta} d \theta+\frac{\partial y}{\partial \lambda} \partial \lambda \\
& d z=\frac{\partial z}{\partial r} d r+\frac{\partial z}{\partial \theta} d \theta+\frac{\partial z}{\partial \lambda} \partial \lambda .
\end{aligned}
$$

By differentiating (3.43) and substituting it into the formula

$$
d s^{2}=d x^{2}+d y^{2}+d z^{2}
$$

one obtains:

$$
d s^{2}=d r^{2}+r^{2} d \theta^{2}+r^{2} \sin ^{2} \theta d \lambda^{2} .
$$

Expression (3.46) expresses the fact that spherical coordinates are orthogonal because: spheres $r=$ constant, cones $\theta=$ constant and plane $\lambda=$ constant intersect each other orthogonally. Moreover, there are no terms with $d r d \theta, d r d \lambda$, and $d \theta d \lambda$. In arbitrary orthogonal coordinates $q_{1}, q_{2}, q_{3}$, the general form of the element of arc is:

$$
d s^{2}=h_{1}^{2} d q_{1}^{2}+h_{2}^{2} d q_{2}^{2}+h_{3}^{2} d q_{3}^{2}
$$

In these coordinates, Laplace's operator can be shown as: 


$$
\Delta V=\frac{1}{h_{1} h_{2} h_{3}}\left[\frac{\partial}{\partial q_{1}}\left(\frac{h_{2} h_{3}}{h_{1}} \frac{\partial V}{\partial q_{1}}\right)+\frac{\partial}{\partial q_{2}}\left(\frac{h_{3} h_{1}}{h_{2}} \frac{\partial V}{\partial q_{2}}\right)+\frac{\partial}{\partial q_{3}}\left(\frac{h_{1} h_{2}}{h_{3}} \frac{\partial V}{\partial q_{3}}\right)\right]
$$

For spherical coordinates $q_{1}=r, q_{2}=\theta, q_{3}=\lambda$. The comparison between (3.46) and (3.47) shows the following relation:

$$
h_{1}=1, h_{2}=r, h_{3}=r \sin \theta \text {. }
$$

Substituting these relations into (3.48), then

$$
\Delta V=\frac{1}{r^{2}} \frac{\partial}{\partial r}\left(r^{2} \frac{\partial V}{\partial r}\right)+\frac{1}{r^{2} \sin \theta} \frac{\partial}{\partial \theta}\left(\sin \theta \frac{\partial V}{\partial \theta}\right)+\frac{1}{r^{2} \sin ^{2} \theta} \frac{\partial^{2} V}{\partial \lambda^{2}}
$$

Performing the differentiations, the expression for Laplace's equation in spherical coordinates is obtained:

$$
\Delta V=\frac{1}{r^{2}} \frac{\partial}{\partial r}\left(r^{2} \frac{\partial V}{\partial r}\right)+\frac{1}{r^{2} \sin \theta} \frac{\partial}{\partial \theta}\left(\sin \theta \frac{\partial V}{\partial \theta}\right)+\frac{1}{r^{2} \sin ^{2} \theta} \frac{\partial^{2} V}{\partial \lambda^{2}}
$$

The solution of Laplace's equation (3.16) may be expressed in a series as follows (HEISKANEN; MORITZ, 1967):

$$
V(r, \theta, \lambda)=\sum_{n=0}^{\infty}\left(\frac{a}{r}\right)^{n+1} \sum_{m=0}^{n}\left(A_{n m} Y_{n m}^{c}+B_{n m} Y_{n m}^{s}\right)
$$

$$
\begin{aligned}
& Y_{n m}^{c}=P_{n m}(\cos \theta) \cos (m \lambda) \\
& Y_{n m}^{s}=P_{n m}(\cos \theta) \operatorname{sen}(m \lambda)
\end{aligned}
$$

where $P_{n m}(\cos \theta)$ is the fully-normalized associated Legendre function, $a$ is a scaling factor (usually equal to the equatorial radius of an adopted mean-Earth ellipsoid) and $Y_{n m}^{c}$ e $Y_{n m}^{s}$ are defined as the surface spherical harmonics. 
Considering (HOFMANN-WELLENHOF; MORITZ, 2006):

$$
\left.\begin{array}{l}
\bar{A}_{n m}=\frac{G M}{r} C_{n m} \\
\bar{B}_{n m}=\frac{G M}{r} S_{n m}
\end{array}\right\}(n \neq 0)
$$

and using the dimensionless coefficients and fully-normalized, expression (3.52) takes the form (BIANCALE, 2002 apud ALMEIDA FILHO, 2009):

$$
V(r, \theta, \lambda)=\frac{G M}{r} \sum_{n=1}^{\infty}\left(\frac{a}{r}\right)^{n+1} \sum_{m=0}^{n} P_{n m}(\operatorname{sen} \theta)\left(C_{n m} \cos m \lambda+S_{n m} \operatorname{sen} \lambda\right)
$$

where the Stokes' coefficients are defined as:

$$
\left\{\begin{array}{l}
C_{n, m} \\
S_{n, m}
\end{array}\right\}=\frac{1}{M a^{n}} \iiint_{Q}\left(2-\delta_{m, 0}\right) \frac{(n-m) !}{(n+m) !} r^{\prime n} P_{n, m}\left(\sin \phi^{\prime}\right)\left\{\begin{array}{l}
\cos m \lambda^{\prime} \\
\sin m \lambda^{\prime}
\end{array}\right\} d m
$$

The coefficients values $C_{n m}, S_{n m}$ (3.54) up to a degree and order $L$ represent what is called "geopotential model". The terms $C_{n, 0}\left(=-J_{n}\right),\left[C_{n, m}, S_{n, m}\right]$ and $\left[C_{n, n}, S_{n, n}\right]$ correspond to the zonal, tesseral and sectorial spherical harmonics. They have different behaviors according to the values of the indexes $n$ (degree) and $m$ (order). If $m=0$, the harmonics consist of the Legendre polynomials only, then they divide the sphere into zones and they are called zonal harmonics. If $m=n$, the sphere is divided into positive and negative sectors, in which case they are called sectorial harmonics. Finally, for any other $m$, the sphere is divided into compartments in which they are alternately positive and negative and are called tesseral harmonics (Figure 3.4). 


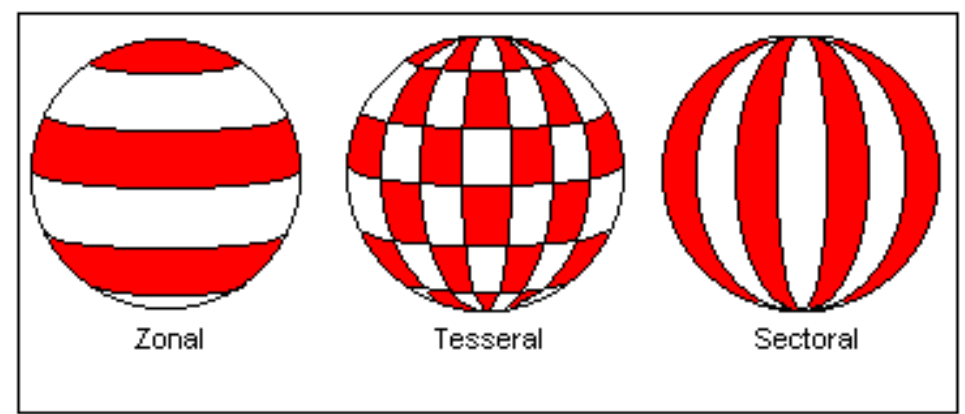

Figure 3.4 - Types of spherical harmonics (Hofmann-Wellenhof; Moritz, 2006).

A Global Gravitational Model is a mathematical approximation to the external gravitational potential of an attracting body (PAVLIS, 1997). A GGM has numerous uses, both operational and scientific, including: orbit determination, inertial navigation, geoid heights computations, oceanographic applications and geophysical prospecting applications.

\subsection{The Geoid and Heights}

The reference surface for heights is usually the geoidal surface, which has constant gravity potential, $W_{0}$, and closely approximates to the mean sea level. If the constant normal gravity potential, $U_{0}$, on the normal ellipsoid is equal to the constant gravity potential of the geoid, then the gravity anomaly on the geoid is the difference between gravity on the geoid and normal gravity on the ellipsoid at respective points, $P_{0}$ and $Q_{0}$. The difference between the geoid and the ellipsoid, $N$, is the geoid height or geoid undulation (Figure 3.5).

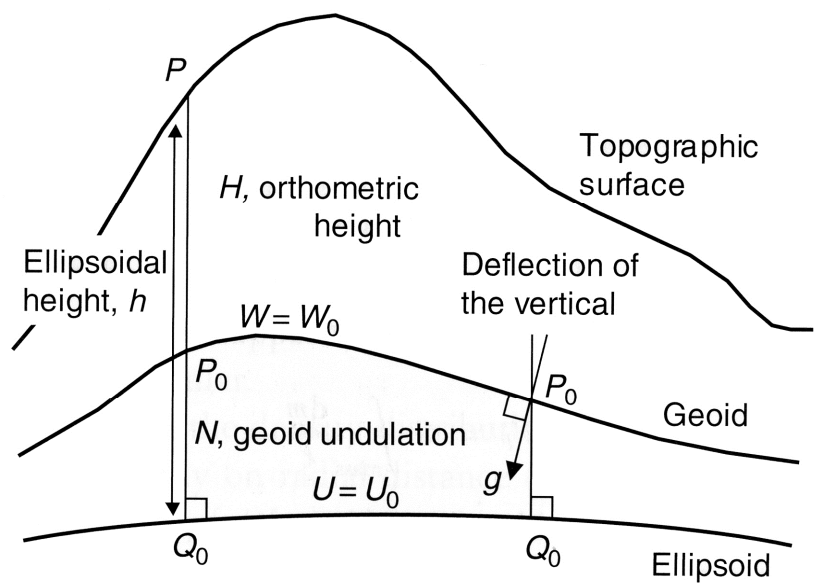

Figure 3.5 - Geodetic quantities (Jekeli, 2009). 
A datum for vertical control over a region or country is defined by a point at mean sea level. In general, the starting point is close to a tide gauge. The determination of gravity potential differences from the initial point to other points on the Earth's surface allows heights with respect to that reference (or in that datum) (JEKELI, 2009). The difference between the gravity potential is known as the geopotential number, at a point $P$, relative to the datum origin $P_{0}$, and it is given by:

$$
C_{P}=W_{0}-W_{P}=\int_{P_{0}}^{P} g d n
$$

where $g$ is gravity, $d n$ is a leveling increment along the vertical direction, and $W_{0}$ is the gravity potential at $P_{0}$.

For these potential differences, various types of height can be defined, for instance, the orthometric height:

$$
H_{P}=\frac{C_{P}}{\bar{g}_{P}}
$$

where

$$
\bar{g}_{P}=\frac{1}{H_{P}} \int_{P_{0}}^{P} g d H
$$

Spirit leveling is a very time consuming operation. Nowadays, geometric vertical position is obtained (with centimeter accuracy or even better) using GPS. The orthometric height can be obtained by first determining ellipsoidal height $h$, from GPS:

$$
H_{P}=h_{P}-N
$$

GPS provides a great economic advantage over the laborious leveling procedure. Thus, this put emphasis on obtaining an accurate geoid height model for land areas. 
Each country or continent specifies a height datum where a local mean sea level is adopted as a datum origin. This can generate some differences between "local data". The comparison involving geoid height from GPS/leveling and geopotential models is a traditional way to evaluate these models and gives a reasonable indication about the geopotential and geoid model accuracy. According to Featherstone et al. (2001), in absolute verifications, GPS network must have been previously tied to a geocentric international terrestrial reference frame. Furthermore, these data can be used to apply constraints on the zero-degree term in order to account for the inexact knowledge of the mass of the Earth and potential of the geoid (HOFMANNWELLENHOF; MORITZ, 2006). Alternatively, GPS/leveling may be used in a relative sense to evaluate the precision of gravimetric geoid or global geopotential model (Featherstone et al., 2001). 


\section{GEOID ESTIMATION}

The geoid height can be computed from several techniques: numerical integration using Stokes' formula directly or using the Fast Fourier Transform, the Least Squares Collocation, spherical harmonic functions developed in series, direct calculation by the difference between the ellipsoidal height (from GNSS) and orthometric height (from spirit levelling), radar altimetry (in the oceans) and combination of them. In this thesis, Stokes' formula applying FFT and LSC were used to compute the geoid model. The first one provides the disturbing potential as a function of the gravity anomalies on the geoid. The use of FFT is to speed up the process when it involves large quantities of data in a discrete way. The second is a mathematical method to determine the anomalous field by a combination of geodetic measurements of different kinds. A modification in the original LSC also can be made to speed up the numerical procedures and this technique is called Fast Collocation.

\subsection{Global Gravitational Models}

A GGM is a set of coefficients of the Earth's gravitational potential function represented in a series of spherical harmonic functions. Rapp (1998) reviewed the past and future developments in geopotential modeling. A GGM consists of a set of numerical values for certain parameters, the statistics of the errors associated with these values (as expressed in their error covariance matrix), and a collection of mathematical expressions and algorithms that allow a user to perform (PAVLIS, 2010):

- Synthesis: compute the numerical values of quantities related to the gravitational potential (functions of the field), given the position of the interest point;

- Error Propagation: compute the expected errors of the computed functionals, as implied by the propagation of the errors of the parameters defining the GGM. 
In addition, the spherical harmonic functions represented by GGM should fulfill certain conditions due to basic physics. It should represent a scalar function of position that is harmonic outside the attracting masses and vanish at infinity as the reciprocal of the distance between attracted point and attracting mass element. Furthermore, the GGM should permit the computation of any functional of the field in a way to guarantee self-consistency. According to Pavlis (2010), a GGM has numerous uses:

1. Orbit determination applications necessary for space surveillance;

2. Inertial navigation use for trajectory resolution of airplanes and missiles;

3. Geoid height computation necessary to transform a geodetic height obtained from GNSS positioning, to an evaluation referenced to an equipotential surface;

4. Oceanographic application in the Dynamic Ocean Topography (DOT) estimation;

5. Geophysical prospecting applications combined with other methodologies.

Terrestrial, marine and airborne gravity data can be used in combination with the GGMs to improve the accuracy and/or resolution of the determination of one or more specific functionals of the anomalous field. Some important points related to the development and to the nature of global and local gravimetric models should be considered (PAVLIS, 2010):

- Data collection, validation and pre-processing is the most time-consuming, expensive and laborious task in the development of both global and local gravimetric models;

- The modeling of GGM, using spherical harmonic functions as the basis, allows the computation of any functional of the anomalous field (geoid heights, gravity anomalies, deflections of the vertical). These components are subjected to commission and omission errors. On the other hand, local and regional gravimetric models usually consist of geographic grids containing the estimated values of one or more specific functional of the field, but cannot support the computation of anomalous field functional at arbitrary locations; 
- Local and regional gravimetric models are seldom accompanied by error statistics computed rigorously from error estimates of the input data, while GGMs are accompanied by increasingly more complete and reliable error estimation;

- The determination of a local/regional model of a given gravimetric functional may be reduced to an interpolation/extrapolation problem, if the functional of interest (gravity anomaly) is also observed directly within the area of interest.

Geodesists considered and undertook the representation of the gravitational potential using point masses (SÜNKEL, 1981; 1983) and finite element methods (MEISSL, 1981; BAKER, 1988). However, these approaches have only limited application. Spherical harmonic functions have prevailed as the standard form used for the representation of the gravitational potential, from the beginning of GGMs determinations, up to the present.

The aim of global high-resolution gravitational modeling is to estimate, as accurately as possible, the coefficients $C_{n m}, S_{n m}$ (3.54), through the combination of gravitational information from a variety of data sources. The estimation of reliable error for $C_{n m}, S_{n m}$ values is also important. The $C_{n m}, S_{n m}$ values can be used to compute the functionals of the anomalous field (geoid height, gravity anomalies, etc.) while their associated errors can be propagated to yield the errors of the derived functionals (PAVLIS, 2010).

\subsubsection{The new satellites missions}

The gravity satellite missions were initialized in the 1990s. The aim was mapping the gravitational field from space with high-resolution. As a result, three satellites were developed: CHAMP (REIGBER et al., 1996), GRACE (GRACE, 1998), and GOCE (ESA, 2006). Table 4.1 shows the main characteristics of the missions. 
Table 4.1 - Main characteristics of the three satellite missions (Adapted from Pavlis, 2010).

\begin{tabular}{|c|c|c|c|c|}
\hline Mission & Status & Orbit & $\begin{array}{l}\text { Mission } \\
\text { Objective }\end{array}$ & $\begin{array}{c}\text { Instrumentation } \\
\text { Tracking and } \\
\text { Comments }\end{array}$ \\
\hline CHAMP & $\begin{array}{l}\text { Launched on } \\
7 / 15 / 2000 \\
\text { Inactive }\end{array}$ & $\begin{aligned} \text { Alt. } & =454 \mathrm{~km} \\
e & \approx 0.004 \\
i & =87^{\circ}\end{aligned}$ & $\begin{array}{l}\text { Gravitational } \\
\text { and magnetic } \\
\text { fields } \\
\text { Atmospheric } \\
\text { Limb sounding } \\
\text { lonosphere } \\
\text { sounding }\end{array}$ & $\begin{array}{l}\text { 3-axis STAR } \\
\text { accelerometer, } \\
\text { GPS and SLR }\end{array}$ \\
\hline GRACE & $\begin{array}{l}\text { Launched on } \\
\text { 3/17/2002 } \\
\text { Active }\end{array}$ & $\begin{aligned} & \text { Alt. }=485 \mathrm{~km} \\
& e \approx 0.001 \\
& i=89^{\circ}\end{aligned}$ & $\begin{array}{l}\text { Gravitational } \\
\text { field and its } \\
\text { temporal } \\
\text { variation }\end{array}$ & $\begin{array}{c}\text { 3-axis } \\
\text { accelerometers } \\
\text { (1 per/GPS) and } \\
\text { SLR K-band } \\
\text { inter-satellite } \\
\text { ranking } \\
\text { Six 3-axis }\end{array}$ \\
\hline GOCE & $\begin{array}{l}\text { Launched on } \\
\text { 3/17/2009 } \\
\text { Active }\end{array}$ & $\begin{array}{c}\text { Alt. } \approx 250 \mathrm{~km} \\
\text { Sun- } \\
\text { synchronous }\end{array}$ & $\begin{array}{c}\text { Gravitational } \\
\text { field (especially } \\
\text { static) }\end{array}$ & $\begin{array}{l}\text { accelerometers } \\
\text { forming the } \\
\text { gradiometer } \\
\text { GPS/GLONNAS } \\
\text { and SLR }\end{array}$ \\
\hline
\end{tabular}

\subsubsection{The GOCE mission}

The GOCE satellite was developed by the European Space Agency and deployed a gravity gradiometry in space to produce homogeneous, highly accurate, near-global maps of the Earth's static gravitational field (e.g. Visser et al., 2001; Drinkwater et al., 2007). It was launched on $17^{\text {th }}$ March 2009, and, according to Rummel (2005) the main objective of the GOCE mission is to obtain a gravitational field model at the $\sim 1$ $2 \mathrm{~cm}$ accuracy level for geoid heights and at the 1-2 mGal level for gravity disturbances and to achieve these results at a spatial resolution better than $100 \mathrm{~km}$. According to the mentioned requirements, the GOCE mission can represent the gravitational potential by spherical harmonic functions completed at least to degree and order 200 , but 250 is envisaged. A summary of the scientific applications of the GOCE data are shown in Figure 4.1. 


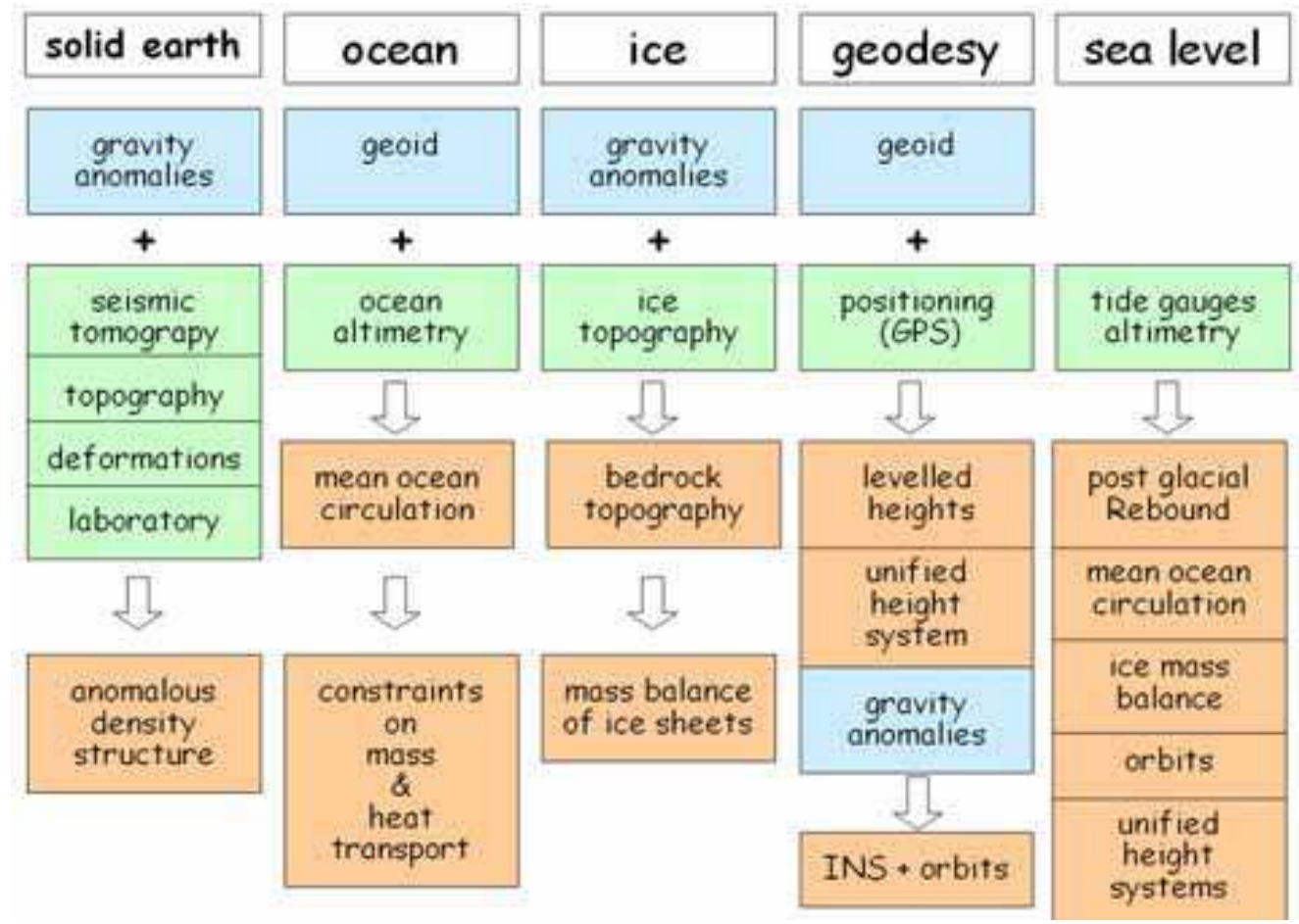

Figure 4.1 - Science applications areas using GOCE data (blue) in conjunction with other satellites, in-situ or other ancillary data (green) (Drinkwater et al., 2007).

The main instrument on GOCE is the Electrostatic Gravity Gradiometer (EGG) shown in Figure 4.2. The EGG consists of three pairs of capacitive accelerometers mounted on a stable support structure. Each accelerometer has a proof mass that is floated in a small cage and it is kept in the centre of the cage by electrostatic forces (by applying some voltage between the cage and the different sides on the parallelipedic shaped mass). This is the initial input for the investigatory steps that, for instance, will determine where the water in the oceans flows (DRINKWATER, 2007; FEHRINGER et al., 2008). 


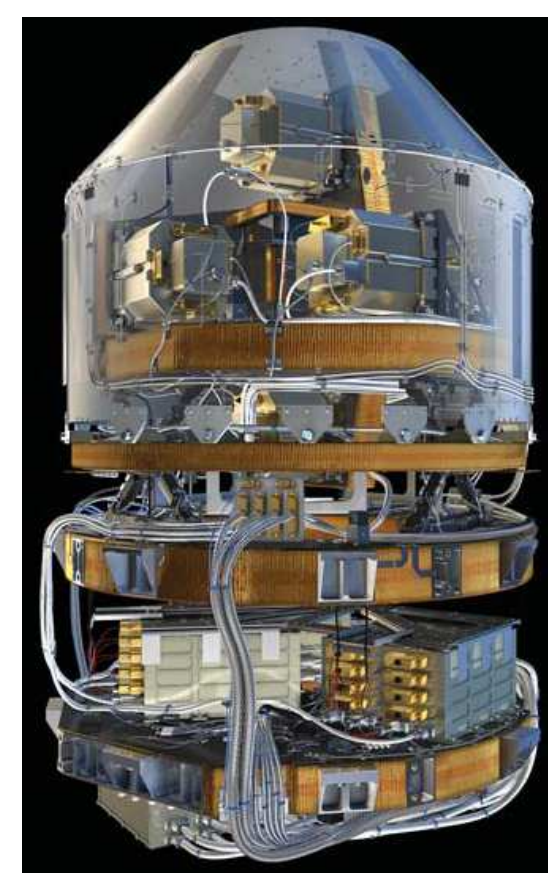

Figure 4.2 - The Electrostatic Gravity Gradiometer (ESA, 2008).

Two accelerometers of the same pair were mounted at a $50 \mathrm{~cm}$ distance apart and form a "gradiometric arm", the distance between each sensor pair must not vary by more than $1 \%$ of an Angstrom (the diameter of an atom) over a mean time interval of about three minutes (FEHRINGER et al., 2008). Under the influence of the Earth's gravitational field, the two proof masses of the same pair have the tendency to move towards or away from each other, and the gradiometer measures this movement.

The results from two accelerometers in one arm are subtracted from each other. The noise and disturbing forces that affect both accelerometers are removed and this is called "common mode rejection". The difference in acceleration due the Earth is what remains and it is called the "gravity gradient". This is the main scientific product of GOCE (DRINKWATER et al., 2007; FEHRINGER et al., 2008). The gradients are obtained in all three dimensions, because the three gradiometric arms are arranged at $90^{\circ}$ to each other. As a result, there is a gravity gradient map covering the Earth except for small areas around the poles. The proof masses have the dimensions of 4 $\mathrm{cm}$ by $4 \mathrm{~cm}$ by $1 \mathrm{~cm}$ and they are made of bulk platinum-rhodium. This shape allows the accelerometer to be tested on ground. The proof mass has the disadvantage of the low accelerometer sensitivity in one direction. The GOCE accelerometer has two ultra-sensitive axis and a third less sensitive axis. The $X Z$ direction was chosen as the remaining ultra-sensitive axes, because this angular rotation is indeed the most 
important in the gravity gradient determination (Figure 4.3). It has a large constant component due to the rotation of the spacecraft around itself (FEHRINGER et al., 2008).

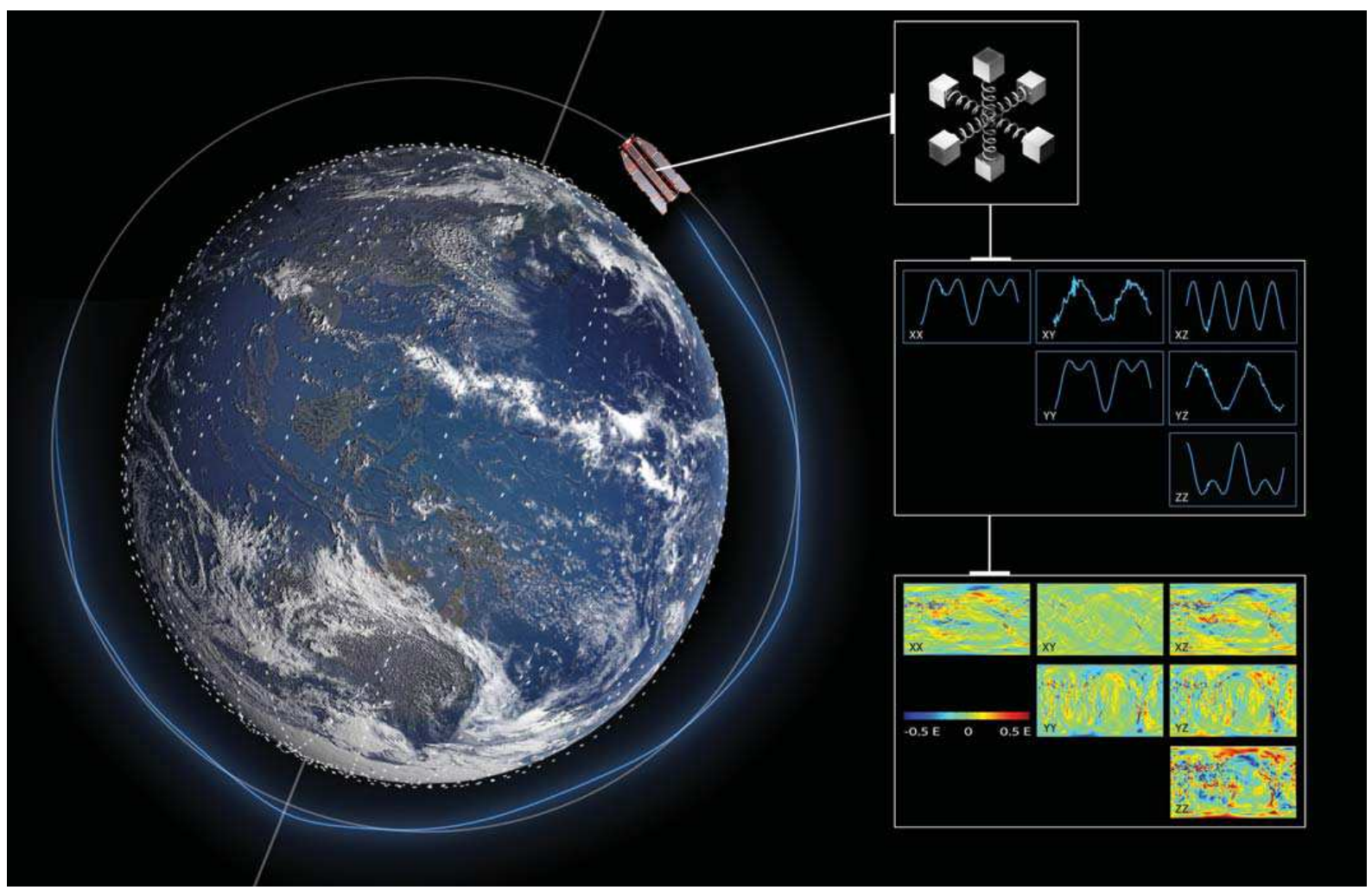

Figure 4.3 - Time series of gravity gradients (upper right). Gravity gradient maps (lower right) (FLOBERGHAGEN et al., 2008).

The Satellite-to-Satellite Tracking Instrument (SSTI) is another important piece of equipment on GOCE. The SSTI is a state-of-the-art GPS receiver that was developed to operate in low-Earth orbit. The aim is to provide support to the gravitational field recovery by using simultaneous tracking of up to 12 GPS satellite signals and works on L1/L2 frequencies (DRINKWATER et al., 2007; FEHRINGER et al., 2008). The SSTI also provides scientific data and real-time information on spacecraft position and velocity for precise orbit determination. According to Fehringer et al. (2008), the receiver uses a hemispherical-coverage quadrifilar helix antenna. To reduce path errors, elevation cut-offs of $5^{\circ}$ and $15^{\circ}$ in the hemispherical coverage are used for the best quality GPS signal and, on average, 8 GPS satellites are visible. 


\subsubsection{Global gravitational models based on GOCE}

Since July 2010, three solutions based on GOCE data have been available to users. The three approaches are as follows: a direct solution (DIR) (BRUINSMA et al., 2010), a time-wise solution (TIM) (PAIL et al., 2010a) and a space-wise solution (SPW) (MIGLIACCIO et al., 2010). The differences between these solutions are the processing strategies applied and the level of a-priori knowledge introduced. Table 4.2 shows their data periods.

Table 4.2 - Data periods of solutions and releases.

\begin{tabular}{cccc}
\hline Solution & From d/m/y & To d/m/y & Number of days \\
\hline DIR_R3 & $01 / 11 / 2009$ & $19 / 04 / 2011$ & 536 \\
DIR_R2 & $01 / 11 / 2009$ & $30 / 06 / 2010$ & 242 \\
DIR_R1 & $01 / 11 / 2009$ & $11 / 01 / 2010$ & 72 \\
TIM_R3 & $01 / 11 / 2009$ & $17 / 04 / 2011$ & 534 \\
TIM_R2 & $01 / 11 / 2009$ & $05 / 07 / 2010$ & 247 \\
TIM_R1 & $01 / 11 / 2009$ & $11 / 01 / 2010$ & 72 \\
SPW_R2 & $30 / 10 / 2009$ & $05 / 07 / 2010$ & 248 \\
SPW_R1 & $30 / 10 / 2009$ & $11 / 01 / 2010$ & 74 \\
\hline
\end{tabular}

The DIR approach is based on the least-squares solution of the inverse problem (PAIL et al., 2011) and all the releases are up to degree and order 240. The data reduction procedure is a combination of the normal equations coming from GPS SSTI observations and normal equations coming from Satellite Gravity Gradiometry (SGG) observations. In the third approach, the GOCE-SGG was fully combined with a GRACE. In order to improve the gravitational field solution, the harmonics of verylow degree (2 and 3) were estimated using LAGEOS-1 and -2 (Laser Geodynamics Satellite) normal equations over the same period as GRACE.

The TIM is the only one based solely on GOCE data. It also has three releases so far, the first one is up to degree and order 224 and the other two up to 250 . This solution considers the gravity gradient and orbit observations (kinematic orbits) as time-series measured along the satellite track. According to Pail et al. (2011), this is especially beneficial considering the highly correlated gravity gradient observations. 
This approach allows evaluating what GOCE can do on its own. The solution may be compared directly with complementary anomalous field information and potential insufficiencies can be detected (PAIL et al., 2011).

The SPW solution has two releases available, (1) up to degree and order 210 and (2) up to 240. It is based on the collocation solution (TSCHERNING, 2001) and the idea is to estimate the spherical harmonic coefficients of the geopotential model by exploiting the spatial correlation of the Earth gravitational field (PAIL et al., 2011). The solution made use of both satellite tracking data derived from the on-board GPS receiver and gravity gradients observed by the on-board electrostatic gradiometer and used kinematic orbits for SSTI gravitational field recovery, while reduced dynamic orbits were used for geo-locating gravity gradients. In order to simplify the notation of the results, the following names will be used: the first three letters represent the solution's name, the letter $R$ followed by a number represents the release (e.g. DIR_R3 is the third release from the direct solution).

The newest EIGEN (European Improved Gravity Model of the Earth by New Techniques) solution is divided into EIGEN-6S and EIGEN-6C (FÖRSTE et al., 2011). The first one is a satellite-only gravitational field of a maximum degree and order 240. It consists of 6.5 years of LAGEOS (SLR) and GRACE (GPS-SSTI and Kband range rate) data and 6.7 months of GOCE (satellite gradiometry). The second solution is a combined global gravitational field of maximum degree and order 1420 . It also used the same data of EIGEN-6S, as well as the DTU2010 (ANDERSEN, 2010) global gravity anomaly data set obtained from altimetry and gravimetry. The solution was obtained from one full normal equation to degree and order (d/o) 365 and a block diagonal solution (for the terrestrial data only) to d/o 1420 .

The GOCO (Gravity Observation COmbination) is an initiative in the frame of ESA's GOCE Data AO, aiming to compute high-accuracy and high-resolution static global gravitational field models. The first solution, GOCO01S (PAIL et al., 2010b), was published with d/o 224 and it used GOCE and GRACE satellite data. The GOCO02S (GOIGINGER et al., 2011) consists of 8 months of GOCE, 7 years of GRACE, 8 years of CHAMP and 5 years of SLR and it was developed up to d/o 250. The last solution, GOCO03S (MAYER-GÜRR, et al., 2012) was computed using 18 months of GOCE gradiometry solution (November 2009 - April 2011), 7 years of GRACE, 8 years of CHAMP and 5 years of SLR and it was developed up to d/o 250. 


\subsubsection{The degree variance of the gravitational models}

The error estimates from the gravitational models are a valuable source of information regarding the quality of the model. In Figure 4.4, the spectra of the signal (upper part) and error (lower part) for seven models (EGM2008, DIR_R2, TIM_R2, SPW_R2, GOCO02S, EIGEN-6S and EIGEN-6C) are shown in terms of gravity anomaly degree variances $\sigma_{l}^{2}(\Delta g)$ and error degree variances $\delta \sigma_{l}^{2}(\Delta g)$ according to Rapp (1973):

$$
\sigma_{n}^{2}(\Delta g)=\gamma^{2}(l-1)^{2} \sum_{m=0}^{l}\left(\bar{C}_{n m}^{2}+\bar{S}_{n m}^{2}\right)
$$

where $\gamma$ is the mean value of normal gravity and $\bar{C}_{2,0}$ and $\bar{C}_{4,0}$ are referenced to an appropriate ellipsoid flattening and

$$
\delta \sigma_{n}^{2}(\Delta g)=\gamma^{2}(l-1)^{2} \sum_{m=0}^{l}\left(m_{\bar{C}_{n m}}^{2}+m_{\bar{S}_{n m}}^{2}\right)
$$

where $m_{\bar{C}_{n m}}, m_{\bar{S}_{n m}}$ are the standard deviation of the harmonic coefficients. 

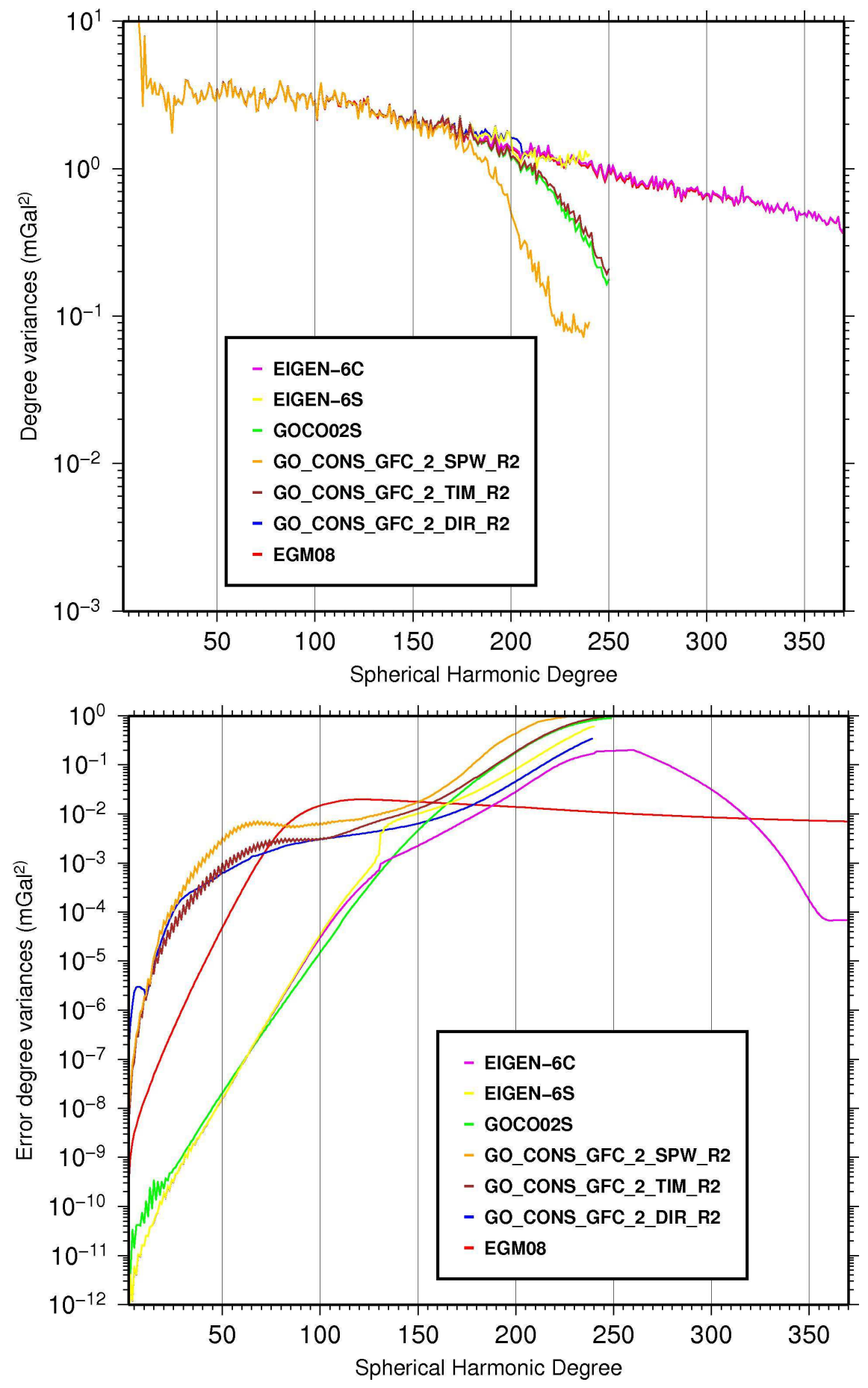

Figure 4.4 - Degree variance (upper) and error degree variances (lower).

The models signal degree variances are very close to degree 170. After that, the corresponding values of GOCO02S present a fast decrease. Therefore, this latter model shows less power in very high frequencies than other models. The differences between the error degree variances of the models are more significant. The error degree variances are representative of the global accuracy of a model which could 
be over or underestimated and reflect different philosophies regarding the weighting of terrestrial and altimetry data (ARABELOS; TSCHERNING, 2010).

The error degree variances of the DIR_R2, TIM_R2 and SPW_R2 are close to the corresponding value of EGM2008 up to degree 180. GOCO02S, EIGEN-6C and EIGEN-6S are very close to 240. The DIR_R2, TIM_R2, SPW_R2, GOCO02S and EIGEN-6S up to $3.5 \times 10^{-1}$ (degree 240), 1.00 (degree 250), $9.94 \times 10^{-1}$ (degree 240), $9.14 \times 10^{-1}$ (degree 250), $6.18 \times 10^{-1}$ (degree 240) $\mathrm{mGal}^{2}$, respectively. EIGEN-6C reaches its highest error $\left(2.02 \times 10^{-1} \mathrm{mGal}^{2}\right)$ in degree 260 (after this degree, the combination scheme of this model adds block diagonal solution of global anomalous field and mean sea surface - DTU10), decays rapidly up to degree 359 and later remains at $10^{-3} \mathrm{mGal}^{2}$ up to degree 1420 , while the corresponding values of the EGM2008 remain at the $10^{-2} \mathrm{mGal}^{2}$ level, almost up to degree 2160 .

The amplitude (solid lines) and commission error (dashed lines) for seven models are represented in Figure 4.5. These errors reflect the uncertainties of the spherical harmonic coefficients, which are due to errors in the observations that propagate in the geoid height. The GGMs error for maximum degree is: $10 \mathrm{~cm}, 16 \mathrm{~cm}, 9 \mathrm{~cm}, 17$ $\mathrm{cm}, 20.5 \mathrm{~cm}$ and $8 \mathrm{~cm}$ for EIGEN 6C, GOCO02S, DIR_R2, TIM_R2 and SPW_R2 and EGM2008, respectively. The EGM2008 commission error for degree 150 is $6 \mathrm{~cm}$.

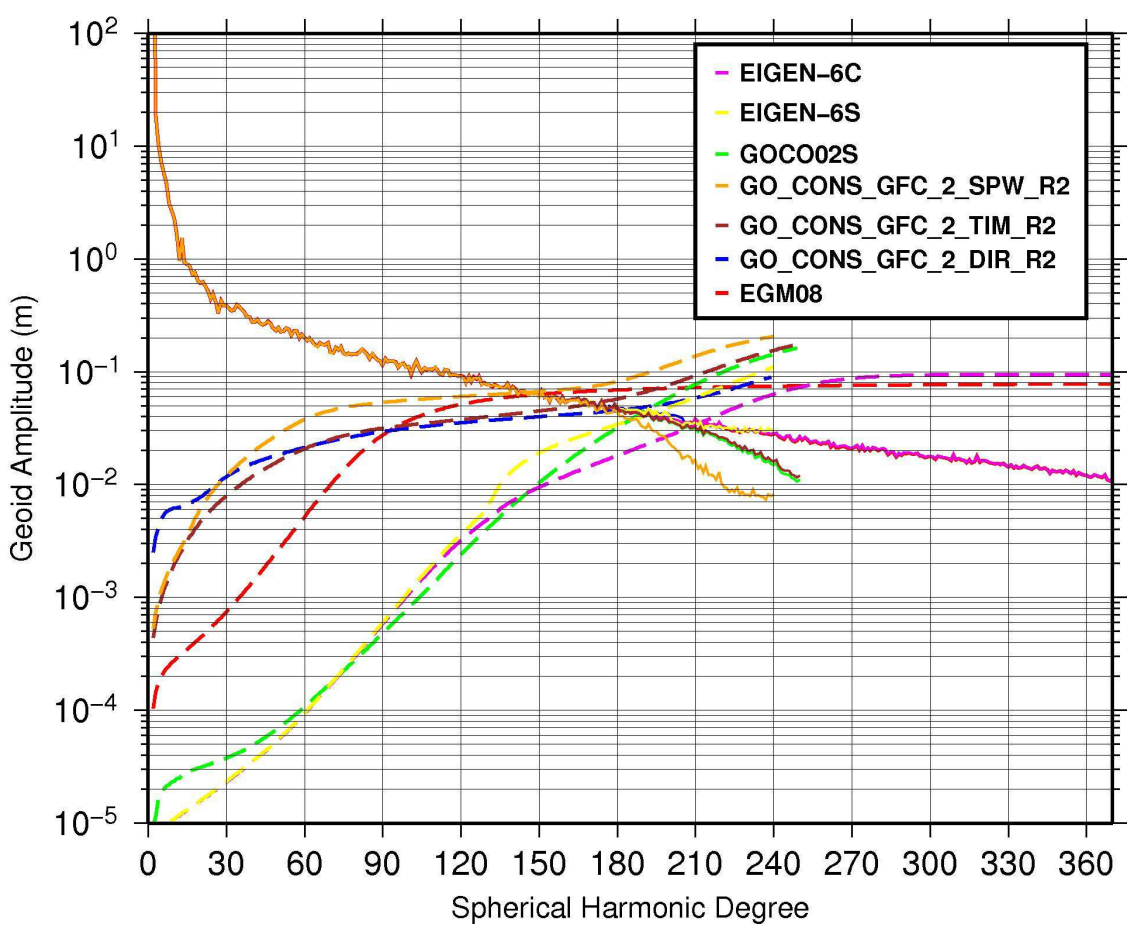

Figure 4.5 - Amplitude and commission error. 


\subsection{Stokes' Integral}

Starting from Pizzetti's formula (HOFMANN-WELLENHOF; MORITZ, 2006):

$$
T(r, \theta, \lambda)=\frac{R}{4 \pi} \iint_{\sigma} S(r, \psi) \Delta g d \sigma
$$

where

$$
S(r, \psi)=\frac{2 R}{l}+\frac{R}{r}-3 \frac{R l}{r^{2}}-\frac{R^{2}}{r^{2}} \cos \psi\left(5+3 \ln \frac{r-R \cos \psi+l}{2 r}\right) .
$$

Then, on the geoid itself, it has $r=R$ and denoting $T(R, \theta, \lambda)$ simply by $T$, one obtains:

$$
T=\frac{R}{4 \pi} \iint_{\sigma} \Delta g S(\psi) d \sigma
$$

where

$$
S(\psi)=\frac{1}{\sin \left(\frac{\psi}{2}\right)}-6 \sin \frac{\psi}{2}+1-5 \cos \psi-3 \cos \psi \ln \left(\sin \frac{\psi}{2}+\sin ^{2} \frac{\psi}{2}\right)
$$

This expression is obtained from $S(r, \psi)$ by setting:

$$
r=R \text { and } l=2 R \sin \frac{\psi}{2}
$$

By Bruns' theorem (3.40), the Stokes' formula reads as follow:

$$
N=\frac{R}{4 \pi \gamma_{0}} \iint_{\sigma} \Delta g S(\psi) d \sigma
$$


where $S(\psi)$ is the Stokes' function.

When Stokes' formula was presented, it was purely theoretical. The reason was the absence of gravimetric measurements over the entire terrestrial surface and especially in the oceans. The ocean gravimetric determinations were initialized from Meinesz (EWING, 1938). A pendulum installed on board of the submarine provided a perspective for the use of this integral. Furthermore, the contribution of relative gravimeters allowed the spread of gravimetric measurements over the continents; nowadays there are still gaps in some regions over the planet, while, in the oceans, the satellite altimeter equated the problem of gravity determination.

In his formulation, Stokes admitted that there are no masses outside the considered equipotential surface, $T$ is assumed to be harmonic outside the geoid. In this case, it should reduce the gravity measurements carried out on the terrestrial surface to the geoidal surface and compensate the masses outside the geoid. This reduction leads to a mass redistribution, which means the creation of a fictitious Earth with a gravity potential changed. The geoid height obtained by Stokes' integral is represented by the separation between the reference ellipsoid and a "fictitious geoid", called cogeoid. The difference between the geoid and the co-geoid it is called indirect effect.

\subsubsection{Stokes' kernel modification}

The geoid model computation using the spectral decomposition combines a global geopotential model with terrestrial gravity observations and a digital terrain model around the area of interest. Formally, as said before, Stokes' formula requires gravity data over the whole Earth. However, the global coverage of gravity data is incomplete. The computation is performed by using only those data that surrounds and includes the area where the geoid is desired. The performance of the residual Stokes integration over a limited area causes a truncation error (also called far-zone contribution of Stokes' integral) in the geoid height.

According to Rapp and Rummel (1975), the gravimetric estimate of the geoid height $(N)$ can be divided into the following two contributions: 


$$
N=N_{L}+\delta N_{L}
$$

where $N_{L}$ is the long-wavelength contribution of the global geopotential model complete to the spherical harmonic degree and order $(L)$, and $\delta N_{L}$ is the residual geoid contribution from Stokes' formula. The modified Stokes' integral can be presented according to (BLITZKOW, 1996):

$$
\delta N_{L}=\frac{R}{4 \pi \gamma} \int_{0}^{2 \pi} \int_{0}^{\pi} \delta S_{L}^{m}(\psi) \delta g_{L} \sin \psi d \psi d \alpha
$$

where $\delta S_{L}^{m}$ is the spherical Stokes integration kernel by a suitable redesign. Without a concern with the deduction that can be found at (VANIČEK et al., 1987 and BLITZKOW; CINTRA; FORTES, 1991), the formulas read as follow:

$$
\delta S_{L}^{m}(\psi)=\delta S_{L}(\psi)-\overline{\delta S}_{L}(\psi)
$$

with

$$
\begin{gathered}
\delta S_{L}(\psi)=S(\psi)-S_{L}(\psi) \\
S_{L}(\psi)=\sum_{n=2}^{1} \frac{2 n+1}{n-1} P_{n}(\psi) \\
\overline{\delta S}_{L}(\psi)=\sum_{n=0}^{1} \frac{2 n+1}{2} t_{n} P_{n}(\cos \psi)
\end{gathered}
$$

In practice, expression (4.9) is usually performed over a limited or truncated spherical cap, which is bound by the surface spherical distance $\psi \leq \psi_{0}$. According to Featherstone, Evans and Olliver (1998), in the region $\psi_{0}<\psi \leq \pi$, the truncation error should be reduced, ideally to zero. If $\psi=0$ the expression converges to infinity. A kernel modification is used effectively to alter the shape of Stokes' kernel such that the truncation error in expression (4.9) is reduced, preferably to the point at which it 
can be safely neglected. The aim of a kernel modification is to reduce the truncation error to a level which is acceptable for modern geodetic applications. Figure 4.6 presents the geoid height components.

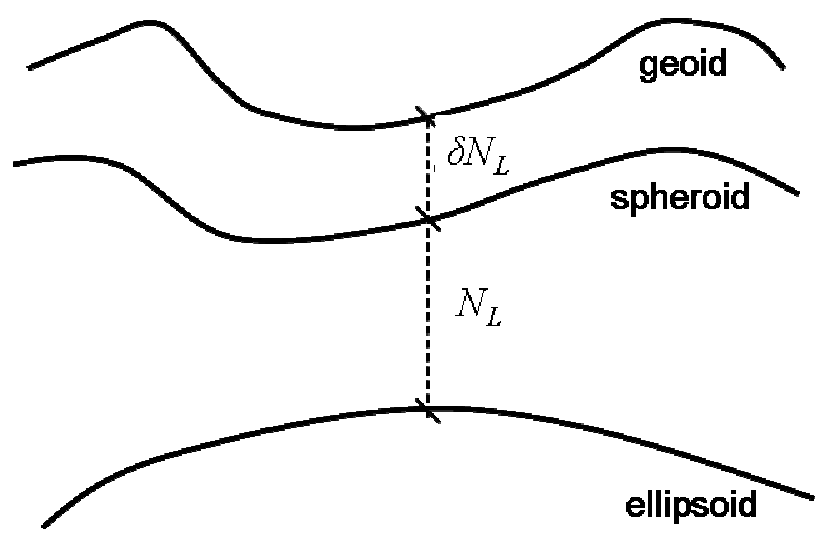

Figure 4.6 - Geoid height components.

According to Molodensky, Eremeev and Yurkina (1962) apud Featherstone, Evans and Olliver (1998) the basis of kernel modification in expression (4.13) is redefined in the region $\psi_{0}<\psi \leq \pi$ as

$$
\overline{\delta S}_{L}(\psi)=\sum_{n=2}^{\infty} \frac{2 n+1}{2} Q_{n}\left(\psi_{0}\right) P_{n}(\cos \psi)
$$

where

$$
Q_{n}\left(\psi_{0}\right)=\int_{\psi_{0}}^{\pi} S(\psi) P_{n}(\cos \psi) \sin \psi d \psi
$$

defines the Molodensky's truncation coefficients.

The theoretical basis for the reduction of the truncation error using modified integration kernels has been investigated by many authors. These include deterministic approaches (MOLODENSKY et al., 1962, WONG; GORE 1969, MEISSL 1971, HECK; GRÜNINGER 1987, VANÍČEK; KLEUSBERG 1987, and VANÍČEK; SJÖBERG 1991) and stochastic approaches (WENZEL 1982, SJÖBERG 1984, SJÖBERG 1991, and VANÍČEK; SJÖBERG 1991). 
Regarding the relevant deterministic kernels, Wong and Gore (1969), proposed to remove the low-degree of the Legendre polynomials $(2 \leq n<L)$ from the Stokes' kernel. By the removing of the low-degree harmonics, the Stokes formula will not distort the long-wavelength contribution to the geoid. The removal of the Legendre polynomials introduces a kernel higher than second-degree and it has been called the spheroidal Stokes' kernel (VANÍČEK; FEATHERSTONE, 1998). This is:

$$
\delta S^{M}(\psi)=S(\psi)-\sum_{n=2}^{M-1} \frac{2 n+1}{n-1} P_{n}(\cos \psi) .
$$

Vaníček and Kleusberg (1987) proposed a modification to the spheroidal Stokes' kernel, which minimizes the upper bound of the truncation error in a root mean square sense. This modification is defined by:

$$
\delta S_{v k}^{M}(\psi)=S^{M}(\psi)-\sum_{n=2}^{M-1} \frac{2 n+1}{2} t_{k}\left(\psi_{0}\right) P_{k}(\cos \psi) .
$$

Meissl (1971) shows that the truncation error series converges to zero faster with increasing spherical harmonic degree $(n)$ when the integration kernel is zero at the truncation radius $\psi_{0}$. Therefore, the effect of the truncation error on the geoid will be reduced at a greater rate when compared with an unmodified kernel (FEATHERSTONE; EVANS; OLLIVER, 1998). The modification is achieved by subtracting the numerical value of the spherical Stokes kernel at the truncation radius $S\left(\psi_{0}\right)$, from the original kernel. The modified kernel is defined as:

$$
S_{m e}(\psi)=S(\psi)-S\left(\psi_{0}\right) \quad \text { for } \quad 0 \leq \psi \leq \psi_{0}
$$

The modified Stokes' integral expression to obtain the geoid height in this thesis (ELLMAN; VANIČEK, 2007) reads as follow: 


$$
\begin{gathered}
N(\Omega)=\frac{R}{4 \pi \gamma_{0}(\phi)} \iint_{\Omega_{\psi 0}} S^{M}\left(\psi_{0}, \psi\left(\Omega, \Omega^{\prime}\right)\right) * \Delta g\left(r_{g}, \Omega\right) d \Omega^{\prime}+\frac{R}{2 \gamma_{0}(\phi)} \sum_{n=2}^{M} \frac{2}{n-1} \Delta g_{n}^{h}\left(r_{g}, \Omega\right)+ \\
+\frac{\delta V^{t}\left(r_{g}, \Omega\right)}{\gamma_{0}(\phi)}+\frac{\delta V^{a}\left(r_{g}, \Omega\right)}{\gamma_{0}(\phi)}
\end{gathered}
$$

where

$$
\Delta g\left(r_{g}, \Omega\right)=\left(\Delta g^{h}\left(r_{t}, \Omega\right)-\sum_{n=2}^{M} \Delta g_{n}^{h}\left(r_{g}, \Omega\right)\right) .
$$

The geocentric position $(r, \Omega)$ of any point is represented by the geocentric radius $r$ and the pair of geocentric coordinates $\Omega=(\varphi, \lambda) ; R$ is the Earth mean radius. In this thesis the modified kernel $S^{M}\left(\psi_{0}, \psi\left(\Omega, \Omega^{\prime}\right)\right)$ proposed by Featherstone (2003) and defined as a combination of the Stokes' kernel modification suggested by Vaniček and Kleusberg (1987) together with Meissl (1971) was used. This kernel presented a better result for the geoid model computation when compared with the spectral decomposition technique without the kernel modification (LOBIANCO, 2005).

Meissl modification proposes the simple subtraction of the Stokes function in relation to the truncation distance, when the error truncation of the Fourier series converges quickly to zero (FEATHERSTONE; SIDERIS, 1998). Vaniček and Kleusberg modification uses the low frequency component of the geoidal undulation obtained by the geopotential model and a spheroid of degree $M$ as a new reference surface (VANIČEK; SJÖBERG, 1991) instead Somigliana-Pizzeti's reference ellipsoid. This modification works in a sense to minimize the truncation error.

In expression (4.19), the first term, on the right side, is the Helmert residual co-geoid. The low degree and order of the reference field is removed before Stokes integration (4.20), then the long-wavelength contribution must be added to the residual component of the geoidal undulation (the second term of the right side in the expression 4.19). The sum of the first two terms results in the Helmert co-geoid. The third term is the primary indirect topographic effect (MARTINEC, 1993), and the last term is the primary indirect atmospheric effect (NOVÁK, 2000). The quasi-geoid is obtained considering the indirect effects. 
The term $\Delta g^{h}\left(r_{t}, \Omega\right)$ on the left hand side in expression (4.20) is the Helmert gravity anomaly referred to the Earth's surface and is given by (VANIČEK et al., 1999):

$$
\Delta g^{k}\left(r_{t}, \Omega\right)=\Delta g\left(r_{t}, \Omega\right)+\delta A^{t}\left(r_{t}, \Omega\right)+\frac{2}{r_{t}(\Omega)} \delta V^{t}\left(r_{t}, \Omega\right)+\delta A^{a}\left(r_{t}, \Omega\right)
$$

The first term on the right hand side in expression (4.21) is the free air anomaly. The second and the third terms are the topographic effects (direct and secondary indirect). The last term is the direct atmospheric effect.

The direct topographic effect $\delta A^{t}\left(r_{t}, \Omega\right)$ is a residual quantity. The direct atmospheric effect $\delta A^{a}\left(r_{t}, \Omega\right)$ is the whole atmospheric gravitational attraction minus the condensed atmospheric gravitational attraction (ELLMAN; VANIČEK, 2007).

\subsubsection{Fast Fourier Transform}

Technological advances, gravity space missions, the search for increasingly precise GGMs, caused an increase in the amount of data. Thus, a greater computational effort and higher processing capacity are required. The quantities used in Geodesy (gravimetric measurements, data derived from radar altimetry, digital terrain models) are presented in a discrete way and the process may involve long intervals of time. One way to overcome this problem is to perform the convolution integrals in the frequency domain, for instance, Stokes' integrals and Vening Meinesz. The fundamental property of these integrals is that they turn into a simple product of functions if its process evaluation is carried out within the frequency domain.

The direct and inverse continuous Fourier transform are called a Fourier transform pair and are defined by (BRACEWELL, 1986; SIDERIS, 2010):

$$
X(f)=\int_{-\infty}^{\infty} x(t) e^{-i 2 \pi t} d t
$$




$$
x(t)=\int_{-\infty}^{\infty} X(f) e^{i 2 \pi t} d f
$$

Expressions (4.22) and (4.23) allow an analytical signal processing in the space domain to the frequency and vice versa.

There are some specific algorithms known as FFT and they process the spectral signal (frequency domain) quickly, solving the Stokes' integral in a regular grid. According to Sideris (2010), using the properties of the Fourier transform, there is no need for time-consuming pointwise solution numerical summation, and the evaluation of convolution integrals is substituted by very efficient multiplications. Moreover, FFT processing gives results in the same grid as the input grid data. When FFT software is processed, it provides geoid height at all points of the gravity anomalies grid. In addition, this technique very successfully overcomes the problem of slow computation speed and provides a homogenous coverage of results, which is very suitable for interpolation and plotting purposes.

The 2-D Fourier transform or spectrum of a function $h(x, y)$ is defined (PAPOULIS, 1977 apud SCHWARZ, SIDERIS, FORSBERG, 1990) by:

$$
H\left(k_{x}, k_{y}\right)=\int_{-\infty-\infty}^{\infty} \int_{-\infty}^{\infty} h(x, y) e^{-i\left(k_{x} x+k_{y} y\right)} d x d y=F[h(x, y)]
$$

where $F$ is the 2-D Fourier operator, $H$ is the spectrum of the function $h(x, y), k_{x}, k_{y}$, are the wavenumbers corresponding to the $x$ and $y$ spatial coordinates respectively, and $i$ is the imaginary unit $(i=\sqrt{-1})$.

Similarly, $F^{-1}$ is the inverse 2-D Fourier operator and the inverse transform is defined by:

$$
h\left(k_{x}, k_{y}\right)=\frac{1}{4 \pi^{2}} \int_{-\infty-\infty}^{\infty} \int^{\infty} H\left(k_{x}, k_{y}\right) e^{i\left(k_{x} x+k_{y} y\right)} d k_{x} d k_{y}=F^{-1}\left[H\left(k_{x}, k_{y}\right)\right] .
$$

The Fourier transform pair is represented schematically by: 


$$
h(x, y) \leftrightarrow H\left(k_{x}, k_{y}\right) \quad \text { or } \quad h(x, y) \leftrightarrow H(u, v)
$$

where $u$ and $v$ are the spatial frequencies (in cycles per distance unit) in the directions of $x$ and $y$ respectively, related to $k_{x}$ and $k_{y}$ by the expressions:

$$
k_{x}=2 \pi u \text { and } k_{y}=2 \pi .
$$

In this way, substituting (4.27) into (4.24) and (4.25), the definition integrals become:

$$
\begin{aligned}
& H(u, v)=\int_{-\infty-\infty}^{\infty} \int_{-\infty}^{\infty} h(x, y) e^{-2 \pi i(u x+v y)} d x d y \\
& h(x, y)=\int_{-\infty-\infty}^{\infty} \int_{-\infty}^{\infty} H(u, v) e^{2 \pi(u x+v y)} d u d v
\end{aligned}
$$

The properties of the Fourier transform will not be discussed in this thesis and can be found in some textbooks, such Papoulis (1977) or Bracewell (1986).

\subsection{Stokes-Helmert Scheme}

\subsubsection{Real space}

Starting from the disturbing potential (3.37) generated by a geocentric reference ellipsoid:

$$
T(r, \Omega)=W(r, \Omega)-U(r, \Omega)
$$

where the geocentric position $(r, \Omega)$ of any point can be represented by the geocentric radius $(r)$ and a pair of geocentric coordinates $\Omega=(\varphi, \lambda)$. 
It is well known that in regions of zero density, the disturbing potential is harmonic. $T$ is harmonic above the geoid surface when topographic and atmospheric masses are completely absent. In this way, Laplace's equation (3.16) is satisfied. Furthermore, if the values of $T$ are known on the geoid surface, then the geoid height can be obtained by Brun's formula (3.40). Since the disturbing potential cannot be measured directly, then the boundary-value problem of the third kind ( $\S 3.2$ ) has to be formulated and solved. To solve this problem, the gravity anomalies, referred to the geoid level, serve as the boundary values (ELLMANN; VANIČEK, 2007). The radial derivative of the disturbing potential is introduced to find a relation between the disturbing potential and the gravity anomalies

$$
\frac{\partial T(r, \Omega)}{\partial r}=\frac{\partial W(r, \Omega)}{\partial r}-\frac{\partial U(r, \Omega)}{\partial r}
$$

Expression (4.31), computed at the Earth's surface, can be approximated by (VANIČEK et al., 1999):

$$
\left.\frac{\partial T(r, \Omega)}{\partial r}\right|_{r=r_{t}} \cong-g\left(r_{t}, \Omega\right)+\gamma\left(r_{t}, \Omega\right)+\varepsilon_{\delta_{g}}\left(r_{t}, \Omega\right)=-\delta g\left(r_{t}, \Omega\right)+\varepsilon_{\delta_{g}}\left(r_{t}, \Omega\right)
$$

where the difference between the actual gravity $g\left(r_{t}, \Omega\right)$ and the normal gravity $\gamma\left(r_{t}, \Omega\right)$ is the gravity disturbance $\delta g\left(r_{t}, \Omega\right)$ and $\varepsilon_{\delta g}\left(r_{t}, \Omega\right)$ is the ellipsoidal correction to the gravity disturbance.

Until the recent past, this quantity was not measurable. Therefore, the gravity disturbance had to be transformed into gravity anomaly. In the modern context, the gravity anomaly is presented by the formula:

$$
\Delta g\left(r_{t}, \Omega\right)=\delta g\left(r_{t}, \Omega\right)+\gamma\left(r_{t}, \Omega\right)-\gamma\left[r_{0}(\varphi)+H^{N}(\Omega)\right]
$$

where $H^{N}(\Omega)$ is the normal height and $r_{0}(\varphi)$ is the geocentric radius of the surface of the reference ellipsoid. 
In expression (4.33), the normal gravity is referred to two different points in the space: the surface and the telluroid. Applying Brun's formula and spherical approximation, this difference becomes (VANIČEK; MARTINEC, 1994):

$$
\begin{aligned}
& \gamma\left(r_{t}, \Omega\right)-\gamma\left[r_{0}(\varphi)+H^{N}(\Omega)\right]=\left.\frac{\partial \gamma(r, \varphi)}{\partial n}\right|_{r=r_{t}(\Omega)} \frac{T\left(r_{t}, \Omega\right)}{\gamma\left[r_{0}(\varphi)+H^{N}(\Omega)\right]}=-\frac{2}{r_{t}(\Omega)} T\left(r_{t}, \Omega\right) \\
& -\varepsilon_{n}\left(r_{t}, \Omega\right),
\end{aligned}
$$

where $\partial \gamma / \partial n$ is the linear approximation of the normal gravity gradient with respect to the normal $n$ of the reference ellipsoid.

Substituting expressions (4.32) and (4.34) into expression (4.33) the fundamental formula of Physical Geodesy (3.34) takes the following form (VANIČEK et al., 1999):

$$
\Delta g\left(r_{t}, \Omega\right)=-\left.\frac{\partial T(r, \Omega)}{\partial r}\right|_{r=r_{t}(\Omega)}+\varepsilon_{\delta g}\left(r_{t}, \Omega\right)-\frac{2}{r_{t}(\Omega)} T\left(r_{t}, \Omega\right)-\varepsilon_{n}\left(r_{t}, \Omega\right)
$$

This expression was formulated for the real space and can be applied in Helmert's space in order to compute Helmert's gravity anomaly $\Delta g^{h}\left(r_{t}, \Omega\right)$. Figure 4.7 presents the quantities involved in real and Helmert's spaces. 


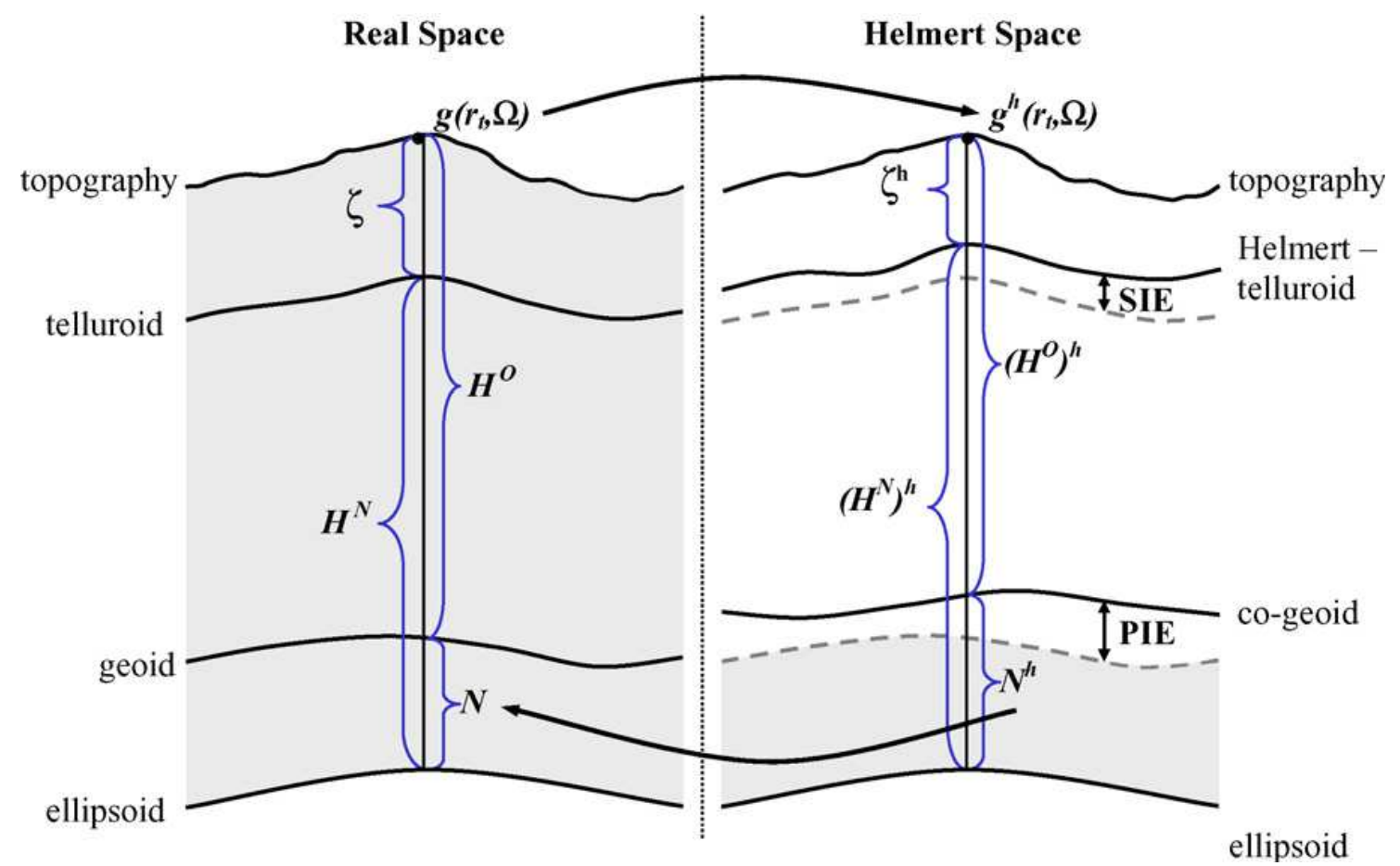

Figure 4.7 - Quantities involved in real and Helmert's spaces (Ellmann; Vaniček, 2007).

\subsubsection{Helmert space}

In the geoid model computation, to satisfy the boundary condition, the topographical masses need to be reduced above the geoid level. Moreover, in order to create a harmonicity of the disturbing potential, atmospheric masses also have to be accounted for. Helmert's second condensation method is applied for this objective. This process defines a new distribution of masses creating a new model Earth, called Helmert's space. This space is distinct from the real Earth space by the difference of the potential of the topographic and atmospheric masses removed and condensed on the geoid. Helmert's gravity potential is defined as follows (ELLMANN; VANIČEK, 2007):

$$
W^{h}(r, \Omega)=W(r, \Omega)-\delta V^{t}(r, \Omega)-\delta V^{a}(r, \Omega)
$$

where superscript $h$ indicates Helmert's space and $\delta V^{t}(r, \Omega)$ is the difference between the potential of the topographical masses and the potential of the condensation layer (residual topographical potential). It is given by: 


$$
\delta V^{t}(r, \Omega)=V^{t}(r, \Omega)-V^{c}(r, \Omega)
$$

and the residual atmospheric potential $\delta V^{a}(r, \Omega)$ is given by:

$$
\delta V^{a}(r, \Omega)=V^{a}(r, \Omega)-V^{c a}(r, \Omega)
$$

where $V^{t}$ is the gravitational potential of the topography removed above the geoid, $V^{c t}$ is the gravitational potential of the condensed layer and $V^{a}$ and $V^{c a}$ are the gravitational potentials of the atmospheric masses removed and condensed.

The disturbing potential in Helmert's space can be written as:

$$
T^{h}(r, \Omega)=W^{h}(r, \Omega)-U(r, \Omega)=T(r, \Omega)-\delta V^{t}(r, \Omega)-\delta V^{a}(r, \Omega)
$$

Applying the analogy in expression (4.35), the relationship between the boundary condition and the Helmert anomaly $\Delta g^{h}\left(r_{t}, \Omega\right)$ is given by:

$$
\begin{aligned}
& \Delta g^{h}\left(r_{t}, \Omega\right)=-\left.\frac{\partial T^{h}(r, \Omega)}{\partial r}\right|_{r=r_{t}(\Omega)}+\varepsilon_{\delta_{g}}\left(r_{t}, \Omega\right)+\left.\frac{\partial \gamma(r, \Omega)}{\partial r}\right|_{r=r_{t}(\Omega)} \frac{T^{h}\left(r_{t}, \Omega\right)}{\gamma\left[r_{0}(\varphi)+\left(H^{N}(\Omega)\right)^{h}\right]} \\
& =-\left.\frac{\partial T^{h}(r, \Omega)}{\partial r}\right|_{r=r_{t}(\Omega)}+\varepsilon_{\delta_{g}}\left(r_{t}, \Omega\right)-\frac{2}{r_{t}(\Omega)} T^{h}\left(r_{t}, \Omega\right)-\varepsilon_{n}\left(r_{t}, \Omega\right) .
\end{aligned}
$$

Helmert gravity anomaly, referred to the Earth's surface can be expressed by (VANIČEK et al., 1999)

$$
\Delta g^{h}\left(r_{t}, \Omega\right)=\Delta g\left(r_{t}, \Omega\right)+\delta A^{t}\left(r_{t}, \Omega\right)+\frac{2}{r_{t}(\Omega)} \delta V^{t}\left(r_{t}, \Omega\right)+\delta A^{a}\left(r_{t}, \Omega\right)
$$

where the first term on the right side is the free-air anomaly, the second and the third terms are the direct and secondary indirect topographical effects. The last term is the direct atmospheric effect. 


\subsection{Least Squares Collocation}

The method of least squares originated from the need to fit a linear mathematical model to given observations. A larger number of measurements than the number of unknown parameters in the model are used to reduce the influence of errors in the observations, solving an overdetermined linear system of equations.

Starting from two sets of random quantities, the set of measurements $l$ and the signals $s$ (MORITZ, 1989):

$$
l=\left[l_{1} l_{2} \ldots l_{q}\right]^{T} \quad \text { and } \quad s=\left[s_{1} s_{2} \ldots s_{m}\right]^{T}
$$

It is assumed that these quantities have an expected value equal to zero. Quantities having mean value zero are called centered. The expectation of the quantities $l$ and $s$ is given by $E\{l\}=0$ and $E\{s\}=0$. Considering the covariance matrices:

$$
\begin{aligned}
& C_{l l}=\operatorname{cov}(l, l), \\
& C_{s l}=\operatorname{cov}(s, l), \\
& C_{s s}=\operatorname{cov}(s, s)
\end{aligned}
$$

where $C_{l l}$ and $C_{s s}$ are the autocovariance matrices of the vectors $l$ and $s$, respectively, and $C_{s l}$ is the cross-covariance matrix between $l$ and $s$.

Considering that the measurement vector $l$ is known and the signal vector $s$ is unknown, the best estimate for $s$ based on $l$ data is a functional relation in terms of the covariance matrices:

$$
\begin{aligned}
& C_{l l}=E\left\{l l^{T}\right\}, \\
& C_{s s}=E\left\{s s^{T}\right\}
\end{aligned}
$$

A linear estimation for the vector $s$ has the form: 


$$
\hat{s}=H l
$$

where $H$ is a $m \times q$ matrix, and each component of the vector $s$ is approximated by a linear combination of data $l$. Error vector $\varepsilon$ is given by $\varepsilon=\hat{s}-s$ and its covariance matrix

$$
C_{\varepsilon \varepsilon}=\operatorname{cov}(\varepsilon, \varepsilon)=E\left\{\varepsilon, \varepsilon^{T}\right\}=E\left\{(\hat{s}-s)(\hat{s}-s)^{T}\right\}
$$

is called error covariance matrix. The best linear estimate of $s$ in terms of $l$ is defined as linear minimum variance estimate (ie. matrix $H$ should be such that the variance errors were minimum):

$$
\hat{s}=C_{s l} C_{l l}^{-1} l
$$

Expression (4.47) is known as the formula of least squares prediction. An example of the least squares prediction can be given considering gravity anomalies:

$$
l=\left[\Delta g_{1} \Delta g_{2} \ldots \Delta g_{q}\right]^{T}
$$

is the gravity anomalies at $q$, and

$$
s=\Delta g_{P}
$$

the gravity anomaly in the interpolation point $P$. Then (4.47) becomes:

$$
\Delta g_{P}=\left[\begin{array}{lll}
C_{P 1} C_{P 2} \cdots C_{P q}
\end{array}\right]\left[\begin{array}{cccc}
C_{11} & C_{12} & \cdots & C_{1 q} \\
C_{21} & C_{22} & \cdots & C_{2 q} \\
\vdots & \vdots & \vdots \\
C_{q 1} & C_{q 2} & \cdots & C_{q q}
\end{array}\right]^{-1}\left[\begin{array}{c}
\Delta_{g 1} \\
\Delta_{g 2} \\
\vdots \\
\Delta_{g q}
\end{array}\right]
$$


The least squares prediction can be applied not only for homogenous data such as gravity anomalies, but to estimate different quantities such as disturbing potential, geoid height and deflections of the vertical.

The least squares collocation is a mathematical method to determine the components of the anomalous field by a combination of geodetic measurements of different kinds. Considering least square prediction discussed above, the quantities (gravity anomaly, deflections of the vertical or gravity disturbances) form vector $l$, and may be represented as a linear function of potential $T$, in a spherical approximation (TSCHERNING 1971; MORITZ, 1989):

$$
\begin{gathered}
\Delta g=-\frac{\partial T}{\partial r}-\frac{2}{R} T \\
\xi=-\frac{1}{\gamma r} \frac{\partial T}{\partial \varphi}, \quad \eta=-\frac{1}{\gamma \sin \varphi} \frac{\partial T}{\partial \lambda} \\
\delta g=-\frac{\partial T}{\partial r}
\end{gathered}
$$

where $r, \varphi, \lambda$ are the spherical coordinates and $\gamma$ is the normal gravity.

$$
l=L T
$$

A linear functional means that $L T$ depends linearly on $T$ according to expressions (4.51), (4.52) and (4.53) but need not be an ordinary function (HOFMANNWELLENHOF; MORITZ, 2006). Suppose that vector $l$ is affected by random measuring errors $n$, and then instead of (4.54) it has:

$$
l=L T+n
$$

Substituting $L T=s$, expression (4.55) becomes as follows:

$$
l=s+n
$$


vector $l$ is decomposed into a "signal" $s$ and "noise" $n$. The signal part represents the gravitational effect and the noise is a synonym for the random measuring errors.

Considering the systematic part and the random part, expression (4.56) becomes as follows:

$$
l=A X+s+n
$$

where $A$ is a $m \times n$ matrix that expresses the effect of parameters $X$ on observations $l$. Figure 4.8 shows the basic mathematical model. Term $A X$ represents a regular and slow curve (it is described by a geopotential model). The problem is to determine curve $A X+s$ by means of discrete observations $l$, which are affected by observational errors $n$.

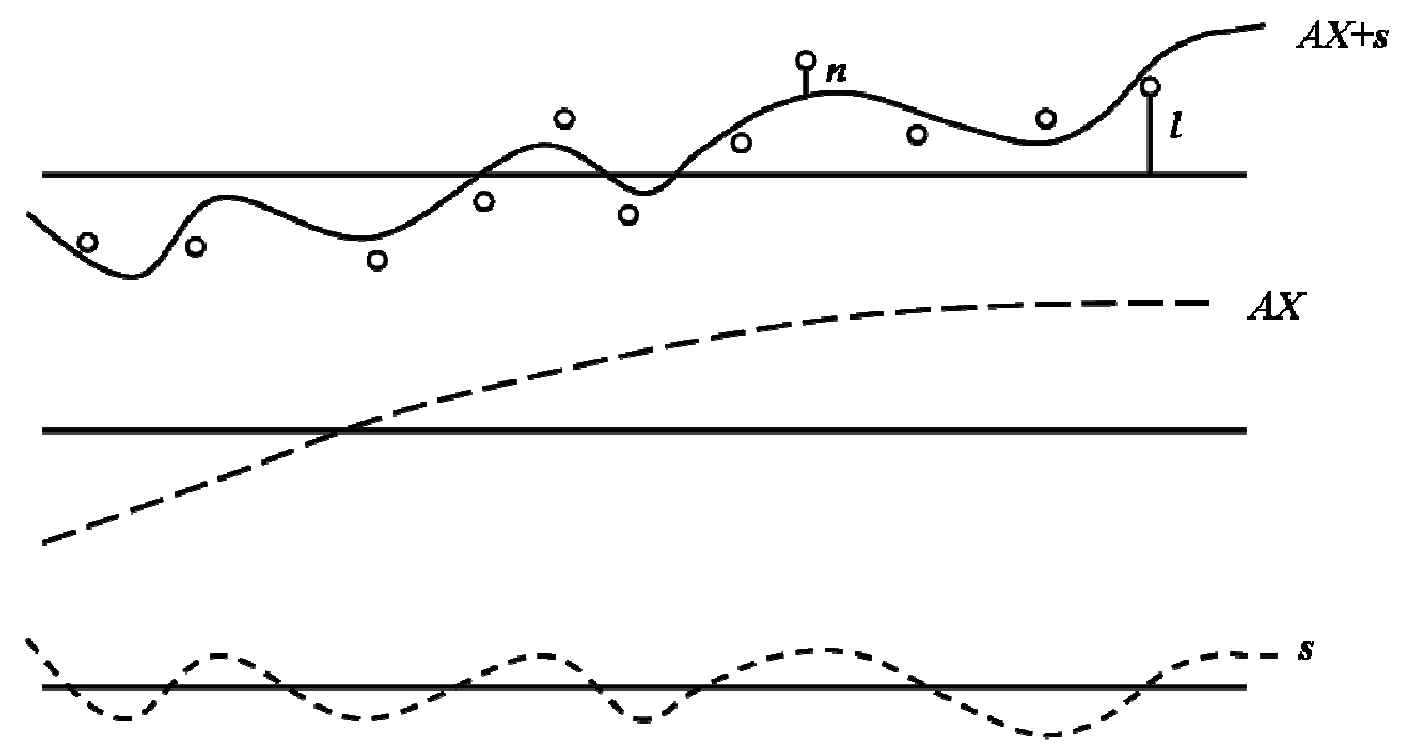

Figure 4.8 - The basic model (MORITZ, 1989).

The anomalous field representation, by heterogeneous data, is solved by applying the uniqueness condition given by:

$$
\alpha\langle T, T\rangle+\beta n^{T} D^{-1} n=\min
$$

and the analytical solution is (MORITZ, 1989): 


$$
\begin{gathered}
\hat{X}=\left[A^{T}(\beta C+\alpha D)^{-1} A\right]^{-1} A^{T}(\beta C+\alpha D)^{-1} I, \\
\hat{T}=(\beta B K)^{T}(\beta C+\alpha D)^{-1}(I-A X)
\end{gathered}
$$

where $\alpha$ and $\beta$ are the numerical parameters, $K$ is the Hilbert's space core, $C$ and $D$ are the symmetric matrices.

As the deterministic component $(A X)$ is represented by a geopotential model (e.g. EGM2008, GOCO03S, EIGEN-6C, etc) expression (4.57) reduces to the non parametric model expressed by expression (4.56). The signal vector solution is represented by expression (4.47) and its respective variance is given by (MORITZ, 1989):

$$
\sigma_{\hat{s}}^{2}=C_{s s}-C_{s t} C_{l l}^{-1} C_{t s}
$$

where $C_{l l}$ is the sum of the signal and the noise covariance matrices $\left(C_{l l}=C_{t t}+C_{n n}\right)$, matrix $C_{s t}$ is the cross covariance matrix and $C_{s s}$ is the covariance matrix for the signal vector $\hat{s}$.

\subsubsection{Covariance function}

The least squares collocation requires all the covariance functions involved in the computation whether a simple estimation (4.61) or the adjustment the solution, of which is given by expression (4.59). The knowledge of the disturbing potential covariance function is essential as the relation between this function and the other covariance functions of the anomalous field (e.g. gravity anomalies, deflections of the vertical, gravity disturbances).

Theoretically, any kind of anomalous field data can be used to obtain the covariance. However, gravity anomalies are more employed once there is a large quantity and the distribution is more homogenous than the other available data. In general, the covariance function reflects the anomalous field behavior describing the variation 
magnitude and roughness. In the statistic point of view, the covariance function features the statistic correlation of two quantities in the anomalous field at two different points.

Considering the disturbing potential at two different points $P(\theta, \lambda)$ and $Q(\theta, \lambda)$, the covariance function of the disturbing potential is defined as:

$$
K(P, Q)=M\{T(P), T(Q)\}
$$

where $M\{\bullet\}$ is the averaging operator. Assuming the nonexistence of the zero order term in the disturbing potential $M\{T\}=0$. Since operator $M$ is homogenous (does not depend on the translation) and isotropic (does not depend on the direction), function $K(P, Q)$ will be a function of spherical distance $\psi$ between $P$ and $Q$ (MORITZ, 1989):

$$
K(P, Q)=K(\psi)=M\{T(P) T(Q)\}=\frac{1}{8 \pi^{2}} \int_{\lambda=0}^{2 \pi} \int_{\theta=0}^{\pi} \int_{\alpha=0}^{2 \pi} T\left(\theta_{P}, \lambda_{P}\right) T\left(\theta_{Q}, \lambda_{Q}\right) \sin \theta d \theta d \lambda d \alpha
$$

where the spherical distance can be express as:

$$
\cos \psi=\cos \theta_{P} \cos \theta_{Q}+\sin \theta_{P} \sin \theta_{Q} \cos \left(\lambda_{P}-\lambda_{Q}\right)
$$

Considering a sphere of unitary radius and the function is harmonic on the sphere, its representation in spherical harmonic is:

$$
T(P, Q)=\sum_{n=2}^{\infty} \sum_{m=0}^{n}\left(\bar{a}_{n m} \cos m \lambda+\bar{b}_{n m} \sin m \lambda\right) P_{n m}(\cos \psi)
$$

$\bar{a}_{n m}, \bar{b}_{n m}$ are the spherical harmonic coefficients associated to the covariance function. The spherical harmonic expansion of the function (4.63) can be written as:

$$
K(\psi)=\sum_{n=2}^{\infty} k_{n} P_{n}(\cos \psi)
$$


where $P_{n}(\cos \psi)$ are the Legendre polynomials and $k_{n}$ can be expressed in terms of $\bar{a}_{n m}$ and $\bar{b}_{n m}$ by:

$$
k_{n}=\sum_{m=0}^{n}\left(\bar{a}_{n m}^{2}+\bar{b}_{n m}^{2}\right)
$$

where $\bar{a}_{n m}$ and $\bar{b}_{n m}$ are coefficients of fully normalized harmonics related to the disturbing potential. The disturbing potential covariance function into the space outside the sphere between points $P$ and $Q$, and situated at a distance $\psi$ is

$$
K(P, Q)=\sum_{n=2}^{\infty} k_{n}\left(\frac{R_{B}^{2}}{r_{P} r_{Q}}\right)^{n+1} P_{n}(\cos \psi)
$$

where $r_{P}, r_{Q}$ are the vector radius of points $P$ and $Q$, and $R_{B}$ is the Bjerhammar radius. Expression (4.68) is by definition a global covariance function.

Tscherning and Rapp (1974) proposed a covariance function model (4.69). This function will be used in this thesis in the sense to fit the empirical covariance function.

$$
K(P, Q)=\sum_{n=2}^{N} k_{n}\left(\frac{R_{B}^{2}}{r_{P} r_{Q}}\right)^{n+2} P_{n}(\cos \psi)+\sum_{n=N+1}^{\infty} \frac{A(n-1)}{(n-2)(n+24)}\left(\frac{R_{B}^{2}}{r_{P} r_{Q}}\right)^{n+2} P_{n}(\cos \psi)
$$

where $N$ is the number truncated in the geopotencial model, $k_{n}$ represents the error degree variance contained in the geopotencial model $R_{B}$ and $A$ are determined via a non-linear adjustment.

\subsubsection{Fast collocation}

Bottoni and Barzaghi (1993) proposed a modification in the original least squares collocation technique in order to speed up the numerical procedures to determine the geoid model. The method, called Fast Collocation, assumes that the input data are 
gridded and homogenous, which implies a particular structure of the covariance matrix. The autocovariance matrix is a symmetric Toeplitz matrix and each block is itself a symmetric Toeplitz matrix (Toeplitz/Toeplitz structure). It is thus possible to compute collocation solutions with large data sets, covering large areas in a single step and in a fast way, without partitioning the data in subsets.

A Toeplitz matrix is a matrix of which each descending diagonal from left to right is constant

$$
T=\left[\begin{array}{ccccc}
t_{0} & t_{1} & t_{2} & \cdots & t_{n-1} \\
t_{-1} & t_{0} & t_{1} & & \\
t_{-2} & t_{-1} & t_{0} & & \vdots \\
\vdots & & & \ddots & \\
t_{-(n-1)} & & & \cdots & t_{0}
\end{array}\right] .
$$

Considering a planar distance $d_{P Q}$ between two points $P$ and $Q$ and the covariance function $C(P, Q)$ in a grid of two dimensions:

$$
C(P, Q)=C(|P-Q|)=C\left(d_{P Q}\right)
$$

In order to clarify the ideas an example in a $3 \times 3$ grid is presented (Figure 4.9). The nodes are numbered in increasing order from left to right in the $x$ component and from the bottom to the top in the $y$ component (BOTTONI; BARZAGHI, 1993). 


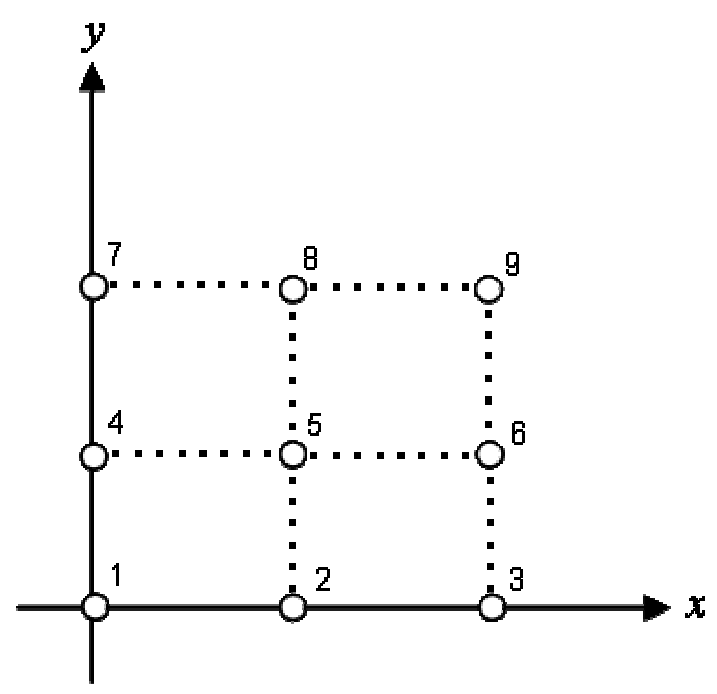

Figure 4.9 - The planar grid.

The computation of this grid using the covariance function (4.71) generates a covariance matrix $C$ which is a $3 \times 3$ symmetric block Toeplitz matrix and each block is itself a $3 \times 3$ symmetric Teoplitz matrix. The $C$ matrix can be written as follows:

$$
C=\left[\begin{array}{ccc}
C_{11} & C_{12} & C_{13} \\
& C_{22} & C_{23} \\
\text { symm } & & C_{33}
\end{array}\right]
$$

and the diagonal blocks are expressed as

$$
\begin{gathered}
C_{11}=\left[\begin{array}{lll}
C\left(d_{11}\right) & C\left(d_{12}\right) & C\left(d_{13}\right) \\
C\left(d_{21}\right) & C\left(d_{22}\right) & C\left(d_{23}\right) \\
C\left(d_{31}\right) & C\left(d_{32}\right) & C\left(d_{33}\right)
\end{array}\right], \\
C_{22}=\left[\begin{array}{lll}
C\left(d_{44}\right) & C\left(d_{45}\right) & C\left(d_{46}\right) \\
C\left(d_{54}\right) & C\left(d_{55}\right) & C\left(d_{56}\right) \\
C\left(d_{64}\right) & C\left(d_{65}\right) & C\left(d_{66}\right)
\end{array}\right], \\
C_{33}=\left[\begin{array}{lll}
C\left(d_{77}\right) & C\left(d_{78}\right) & C\left(d_{79}\right) \\
C\left(d_{87}\right) & C\left(d_{88}\right) & C\left(d_{89}\right) \\
C\left(d_{97}\right) & C\left(d_{98}\right) & C\left(d_{99}\right)
\end{array}\right] .
\end{gathered}
$$


Each matrix listed above is a symmetric Toeplitz matrix since $C\left(d_{i j}\right)=C\left(d_{i j}\right), d_{j j}=0$ and $d_{i+j, j+1}=d_{i j}$. The points in the same row in these matrices are correlated, hence, in a regular grid and considering the property in expression (4.70) it is possible to affirm that

$$
C_{11}=C_{22}=C_{33}=C_{0} .
$$

Considering now the two extradiagonal matrices $C_{12}$ and $C_{23}$

$$
\begin{gathered}
C_{12}=\left[\begin{array}{lll}
C\left(d_{14}\right) & C\left(d_{15}\right) & C\left(d_{16}\right) \\
C\left(d_{24}\right) & C\left(d_{25}\right) & C\left(d_{26}\right) \\
C\left(d_{34}\right) & C\left(d_{35}\right) & C\left(d_{36}\right)
\end{array}\right], \\
C_{23}=\left[\begin{array}{lll}
C\left(d_{47}\right) & C\left(d_{48}\right) & C\left(d_{49}\right) \\
C\left(d_{57}\right) & C\left(d_{58}\right) & C\left(d_{59}\right) \\
C\left(d_{67}\right) & C\left(d_{68}\right) & C\left(d_{69}\right)
\end{array}\right] .
\end{gathered}
$$

Matrix $C_{12}$ is a Toeplitz symmetric matrix because $d_{j, j+3} \quad j=1, \ldots, 3$ are equal to the distance between the first and the second row and the diagonal elements are themselves equal; furthermore $d_{i j}=d_{j i}$ and $d_{15}=d_{26}$. The same relation can be made for $C_{23}$ to prove it. Consequently, one has $C_{12}=C_{23}=C_{1}$. Finally, block $C_{13}$ describes the correlations between points of the first and the third row. The same reasoning used for $C_{12}$ and $C_{23}$ can state that $C_{13}$ is a symmetric Toeplitz matrix and $C_{13}=C_{2}$ (BOTTONI; BARZAGHI, 1993). Thus, matrix $C$ can be rewritten in the following form:

$$
C=\left[\begin{array}{ccc}
C_{0} & C_{1} & C_{2} \\
& C_{0} & C_{1} \\
\text { symm } & & C_{0}
\end{array}\right] .
$$


The reduction of the storage area for matrix $C$ is an important consequence of the Toeplitz/Toeplitz structure. When only the first row of the matrix is stored, the elements of the first block row can be computed since each block is a Toeplitz matrix; then, any other elements of $C$ can be derived using the Toeplitz/Toeplitz structure. This is basic when working with a large amount of data (BOTTONI; BARZAGHI, 1993).

The formulations presented so far are referred to a rectangular grid $(x, y)$ and the covariance function depends on the planar distance between points. From now on, the formulation for a spherical case that is most relevant in Geodesy will be presented. The data are on a regular geographical grid $(\varphi, \lambda)$ and covariance functions $C(\psi)$ depend on the spherical distance (4.63). In the spherical case, the covariance matrix is not the type described before; only the Toeplitz structure of each block is preserved. Figure 4.10 shows that the blocks are not preserved.

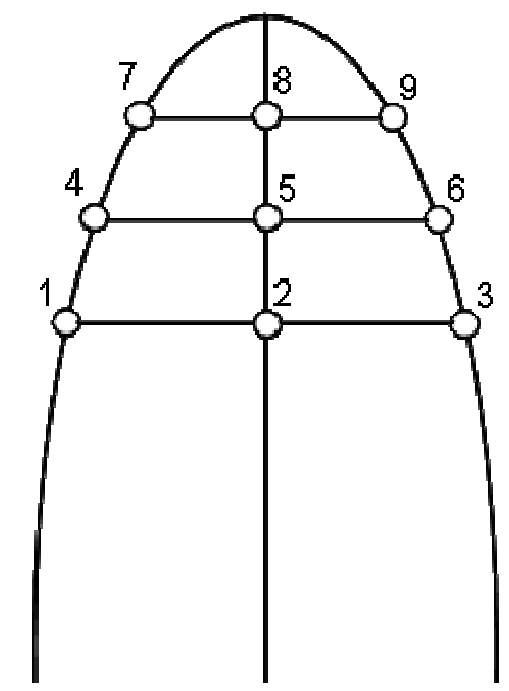

Figure 4.10 - The geographic grid.

The covariances of block $C_{11}$ differ from covariances of block $C_{33}$ and, for these points, argument $f$ of the covariance function is:

$$
\cos \psi_{P Q}=\cos ^{2} \varphi_{P} \cos (n \Delta \lambda)+\sin ^{2} \varphi_{P}
$$

where $\Delta \lambda$ is the grid increment in longitude and $n=\left(\lambda_{P}-\lambda_{Q}\right) / \Delta \lambda$. 
On the other hand, the diagonal of corresponding blocks (e.g. $C_{12}$ and $C_{23}$ ) are equal since they are the covariance between points on different rows but the same meridian. For these points:

$$
\cos \psi_{P Q}=\cos (m \Delta \varphi)
$$

where $\Delta \varphi$ is the grid increment in latitude and $m=\left(\varphi_{P}-\varphi_{Q}\right) / \Delta \varphi$. On the geographical grid, the covariance function $C\left(\psi_{P Q}\right)$ leads to a covariance matrix:

$$
C=\tilde{C}+\delta C
$$

where $\tilde{C}$ is the covariance matrix computed following the Toeplitz/Toeplitz rule and $\delta C$ has zero elements on each block diagonal:

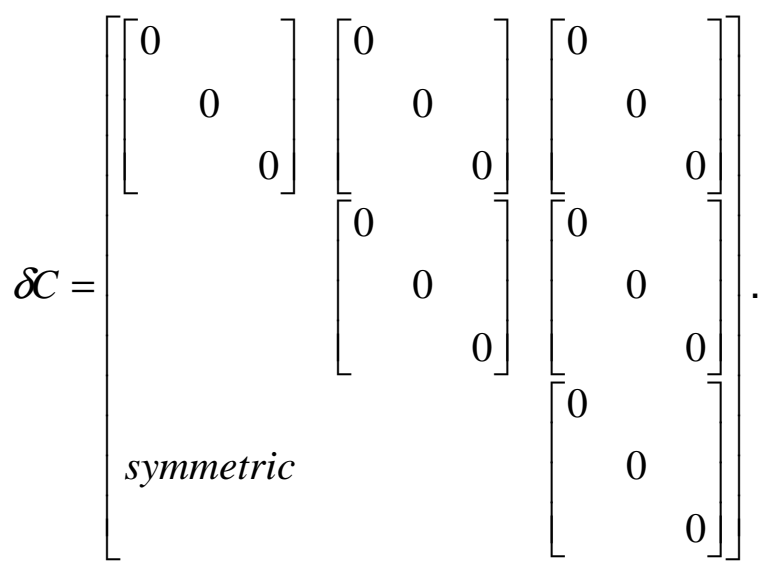

To solve the Toeplitz/Toeplitz system, several numerical tools have been proposed. Levison (1947) and Durbin (1960) developed algorithms to solve a Toeplitz system, while Trench (1964) proposed an algorithm to invert a Toeplitz matrix. Bottoni and Barzaghi (1993) developed an algorithm based on the synergetic application of FFT and the Preconditioned Conjugate Gradient Method (PGGM), which FFT is only used to speed up the procedure. 


\subsection{The Spectral Decomposition (Remove-Restore)}

In the geoid model computation, the anomalous field can be decomposed into three parts: the low-frequencies of the gravity spectrum are obtained from satellite-based global geopotential models (long wavelength); the medium-frequencies are achieved from terrestrial and marine gravity observations (medium wavelength); and the highfrequencies come from topography data (short wavelength). The frequency contributions are shown in Figure 4.11.

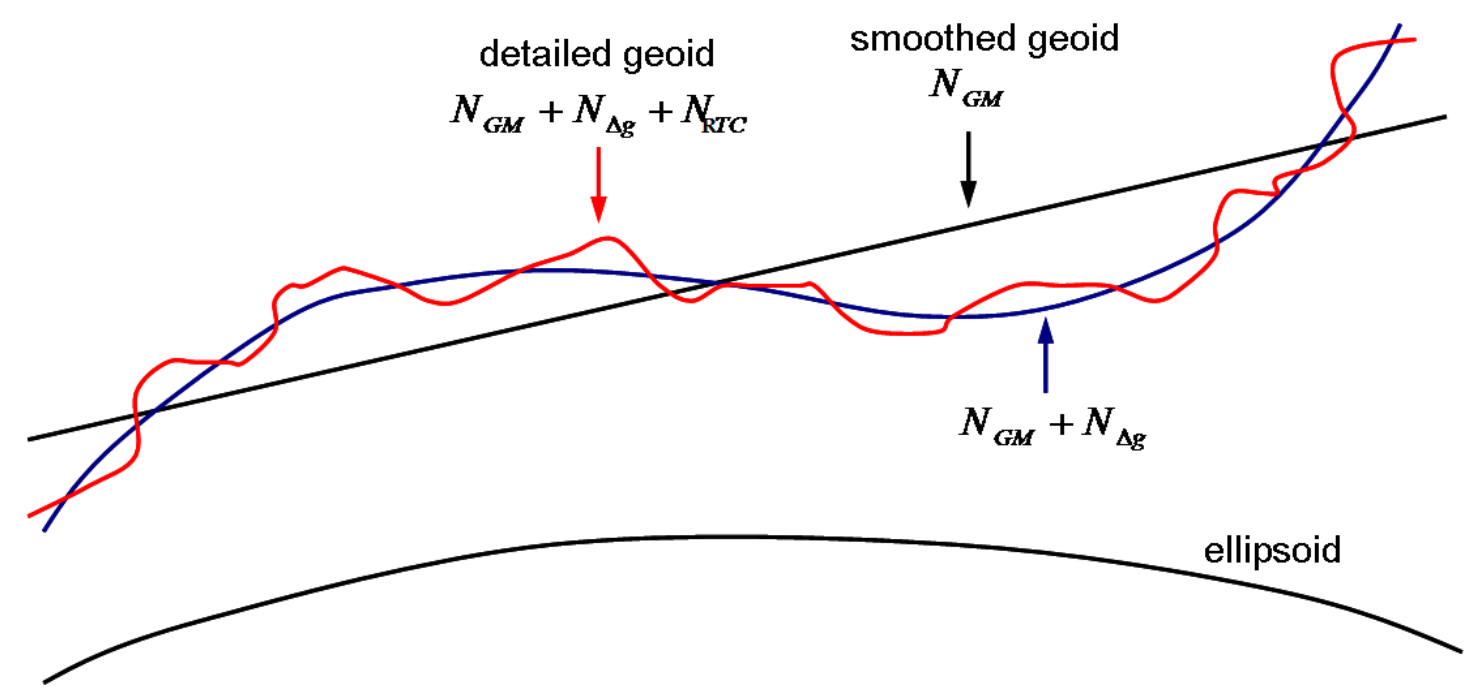

Figure 4.11 - Contributions of different data to regional geoid determination (after Schwarz; Sideris; Forsberg, 1987).

The computation of geoid height $N$ by combining a GGM, mean free air gravity anomalies $\Delta g_{F A}$ and the Residual Terrain Correction (RTC) from a digital terrain model is based on the following formulas:

$$
\begin{gathered}
\Delta g=\Delta g_{F A}-\Delta g_{G M}-\Delta g_{R T C}, \\
N=N_{G M}+N_{\Delta g}+N_{R T C}
\end{gathered}
$$

According to Sideris (2010), geoid heights are more sensitive to low to medium frequencies of the field however, all three data sets are necessary for estimating $N$. 
The gravity anomalies used in Stokes' formula have the contributions of the topography and the GGM removed. The removing process involves the computation and the removal of the GGM and terrain contributions from free-air gravity anomalies, while the restoring step involves the restoration of the GGM contribution and the terrain contribution to $N$ via the indirect effect term. 


\section{DATA SET}

The State of São Paulo was chosen in order to compute the geoid model using different techniques. Figure 5.1 presents the study area delimited by a red square. This area includes the State of São Paulo, as well as some of its and surroundings, and extends from $26^{\circ}-19^{\circ}$ South in latitude and $54^{\circ}-44^{\circ}$ West in longitude. The blue square represents the gravity data area and it is limited by $28^{\circ}-17^{\circ}$ South in latitude and $56^{\circ}-42^{\circ}$ West in longitude. The green square is about the Digital Terrain Model (DTM) and the Digital Bathymetric Model (DBM) and it is one degree larger than gravity area.

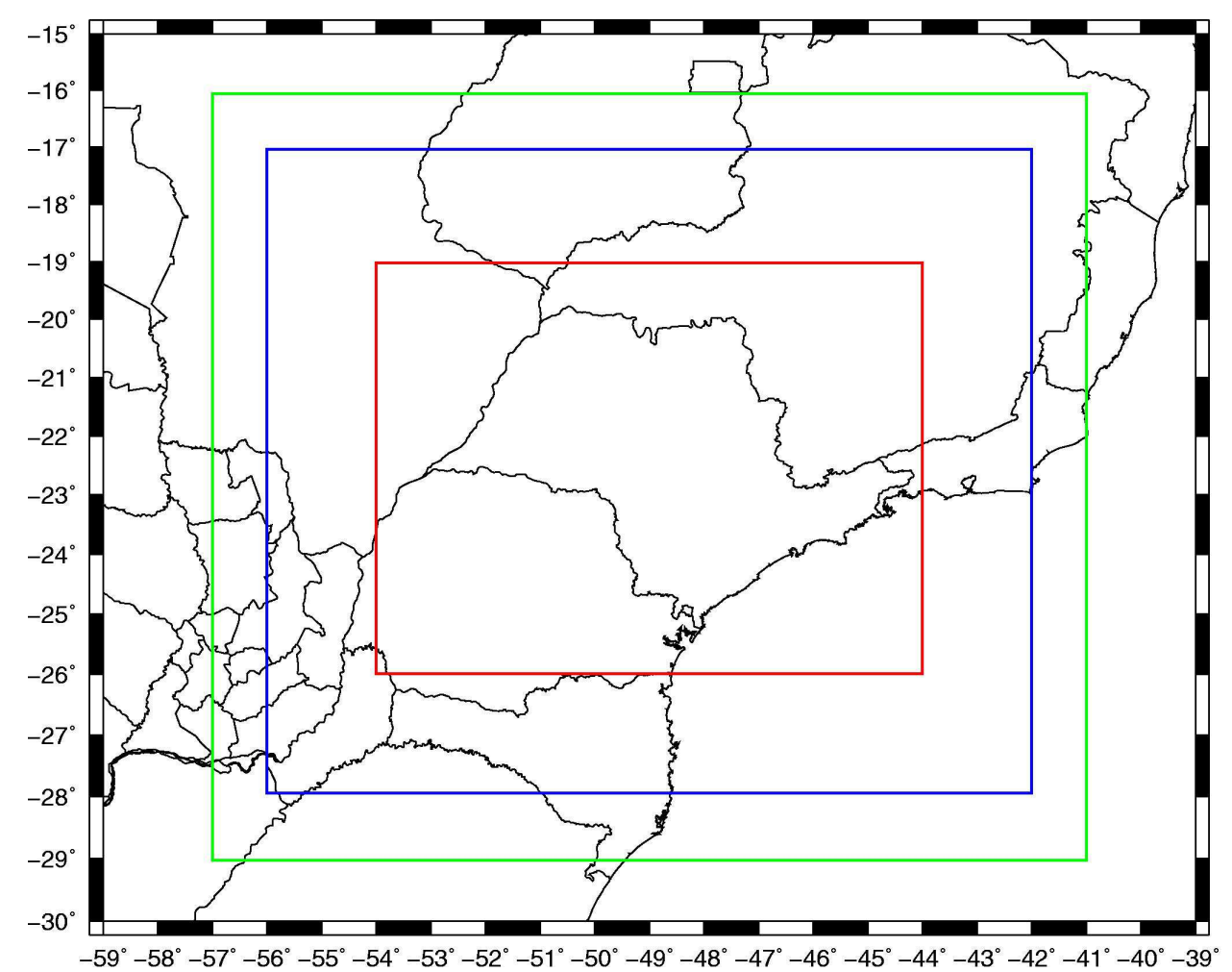

Figure 5.1 - Data area (in red the geoid area, in blue the gravity data and in green the DTM and DBM).

\subsection{Gravity Data Base}

The gravity observations on land and sea represent the most essential source of information of the Earth's gravity field and its internal density distribution. The terrestrial gravity observations carried out with absolute and relative gravimeter form 
the basic data source for evaluating short wavelength of the Earth's gravity field (HECK, 1990).

In this thesis, the Brazilian national gravity data set (BLITZKOW et al., 2010) was used. The study area consists of 46,290 stations (Figure 5.2) and was kindly provided by Observatório Nacional (ON), Brazilian Oil Company (PETROBRAS), Instituto Brasileiro de Geografia e Estatística (IBGE), Instituto de Astronomia, Geofísica e Ciências Atmosféricas (IAG), and Escola Politécnica da Universidade de São Paulo (EPUSP). It is worth mentioning that FAPESP thematic project contributed significantly towards the availability of the terrestrial gravity data used, especially in the State of São Paulo. Subsets also cover neighboring countries (Paraguay and Argentina). With an area of more than one million $\mathrm{km}^{2}$ covered, it is large enough to provide feedback on the GOCE models. The accuracy of the Brazilian terrestrial gravity data is $0.1 \mathrm{mGal}$ level or better (BLITZKOW et al., 2010). In some parts of the area, gravity data resolution is about 5-8 km (São Paulo, Paraná and Santa Catarina states). In the northwest and northeast, the resolution is about $10 \mathrm{~km}$ and there are some gaps. The gravity information was validated by a package dedicated to the validation of gravity data called DIVA developed by Bureau Gravimétrique International (BGI). In the ocean DTU10 (ANDERSEN, 2010) was used. This model is an update of DNSC08 (Danish National Space Center 2008) and it is a truly global gravity field with 1-2 km resolution grid.

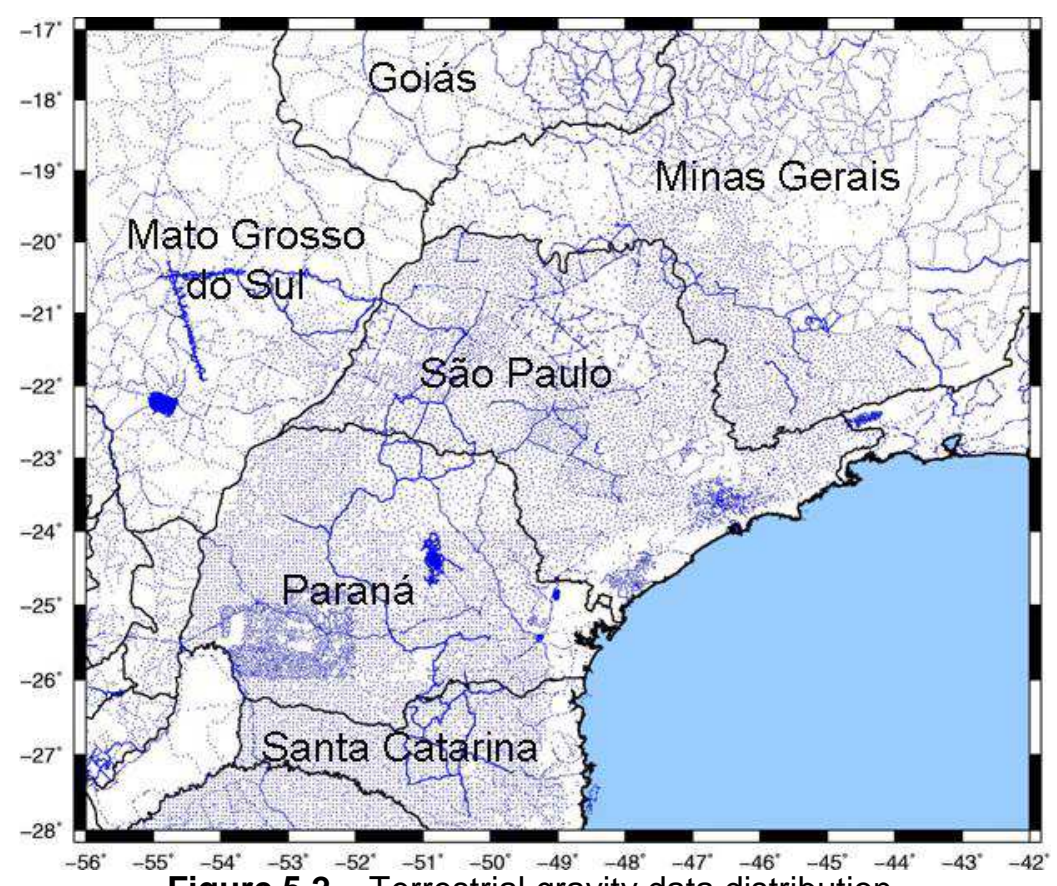

Figure 5.2 - Terrestrial gravity data distribution. 


\subsection{Geopotential Models}

The Global Gravitational Models expressed a substantial function in the geoid determination. They are responsible for the long wavelength information of the gravity field. In this thesis it was used GOCE-based models (discussed in the Chapter 4) and EGM2008.

The first EGM model was developed by the NGA (National Geo-spatial Intelligence Agency) and the GSFC (Goddard Space Flight Center) efforts in the 90's. The EGM96 (LEMOINE et al., 1998a) and (LEMOINE et al., 1998b) has degree and order up to 360 , resolution of 30' and 0.5-1.0 m of precision. In 2008 was presented under the NGA/GSFC supervision the EGM2008 (PAVLIS et al., 2008). This model is complete to degree and order 2159 in ellipsoidal harmonics. Conversion from ellipsoidal to spherical harmonics preserves the order but not the degree. This is why the model extends to degree 2190 but order 2159 . The model has an expected resolution of $0.15 \mathrm{~m}$. EGM2008 has a combined solution based in 57 weeks of GRACE satellite and gravity anomaly data. It was created a database composed by terrestrial, marine and airbone gravity data, beyond gravity anomalies derived by radar altimetry. Figure 5.3 shows data sources used in the EGM2008.

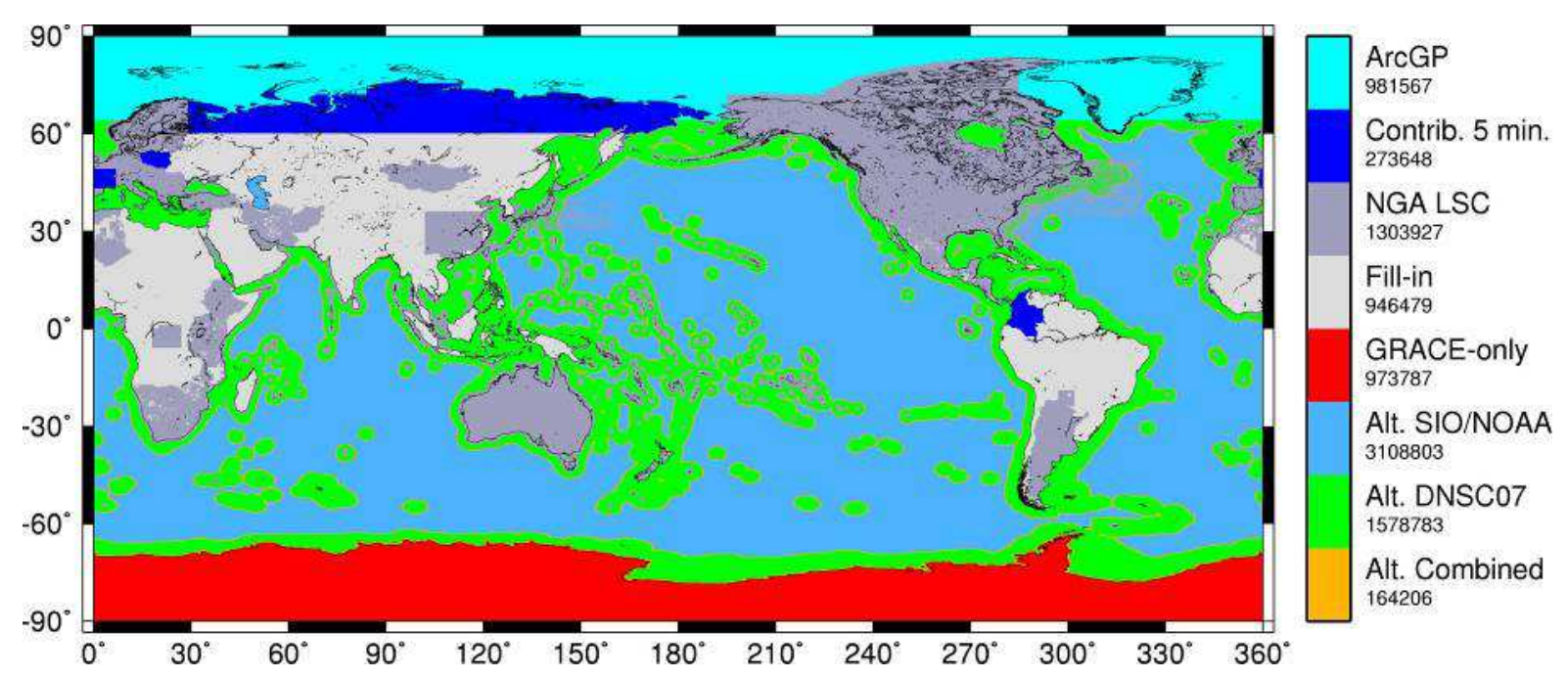

Figure 5.3 $-\Delta$ g data source with $5^{\prime}$ resolution (Holmes; Pavlis, 2008).

The gravity anomalies derived from the altimetry present a homogenous dataset. However, terrestrial data contains some gaps (Antarctica, some parts of Africa, South 
America and Asian Southeast). EGM2008 is referred to the WGS84 (World Geodetic System 1984) and adopted the Tide Free system. SRTM (Shuttle Radar Topographic Mission) and other sources were used to develop a global altimetric model with 30" resolution. A total of 29 different data source was employed in the digital terrain model. This was the greatest advance in relation to the EGM96. Concerning the Mean Sea Level (MSL), DNSC published a MSL model from radar altimetry data. The advances in modeling and altimetry satellite orbits corrections resulted in an improvement relative to EGM96.

EGM2008 evaluation (Table 5.1) involved GPS/leveling data, deflection of the vertical, altimetric TOPEX and GRACE data. It is worth mentioning that GPS/leveling and deflections of the vertical data were not used in the EGM2008 development.

Table 5.1 - GPS/leveling standard deviation.

\begin{tabular}{ccccc}
\hline & \multicolumn{3}{c}{ Total of points = 12,387 } \\
\cline { 2 - 5 } Geopotential model & \multicolumn{2}{c}{ Bias removed } & \multicolumn{2}{c}{ Trend removed } \\
\cline { 2 - 5 } (N maximum) & $\begin{array}{c}\text { edited } \\
\text { numbers }\end{array}$ & $\begin{array}{c}\text { Standard } \\
\text { deviation } \\
\text { (cm) }\end{array}$ & $\begin{array}{c}\text { edited } \\
\text { numbers }\end{array}$ & $\begin{array}{c}\text { Standard } \\
\text { deviation } \\
\text { (cm) }\end{array}$ \\
\hline EGM96 (360) & 12,220 & 30.3 & 12,173 & 27.0 \\
GGM02C_EGM96 (360) & 12,305 & 25.6 & 12,258 & 23.2 \\
EIGEN-GL04C (360) & 12,299 & 26.2 & 12,252 & 23.5 \\
EGM2008 (360) & 12,329 & 23.0 & 12,283 & 20.9 \\
EGM2008 (2190) & 12,352 & 13.0 & 12,305 & 10.3 \\
\hline
\end{tabular}

Source: (Pavlis et al., 2012).

\subsection{Digital Terrain Model}

For the present study, a suitable gridded topography with a grid size of 3" $x 3$ " (approximately $90 \mathrm{~m}$ x $90 \mathrm{~m}$ ) from SAM3s_v2 (MATOS; BLITZKOW, 2008) was used and it is shown in Figure 5.4. 


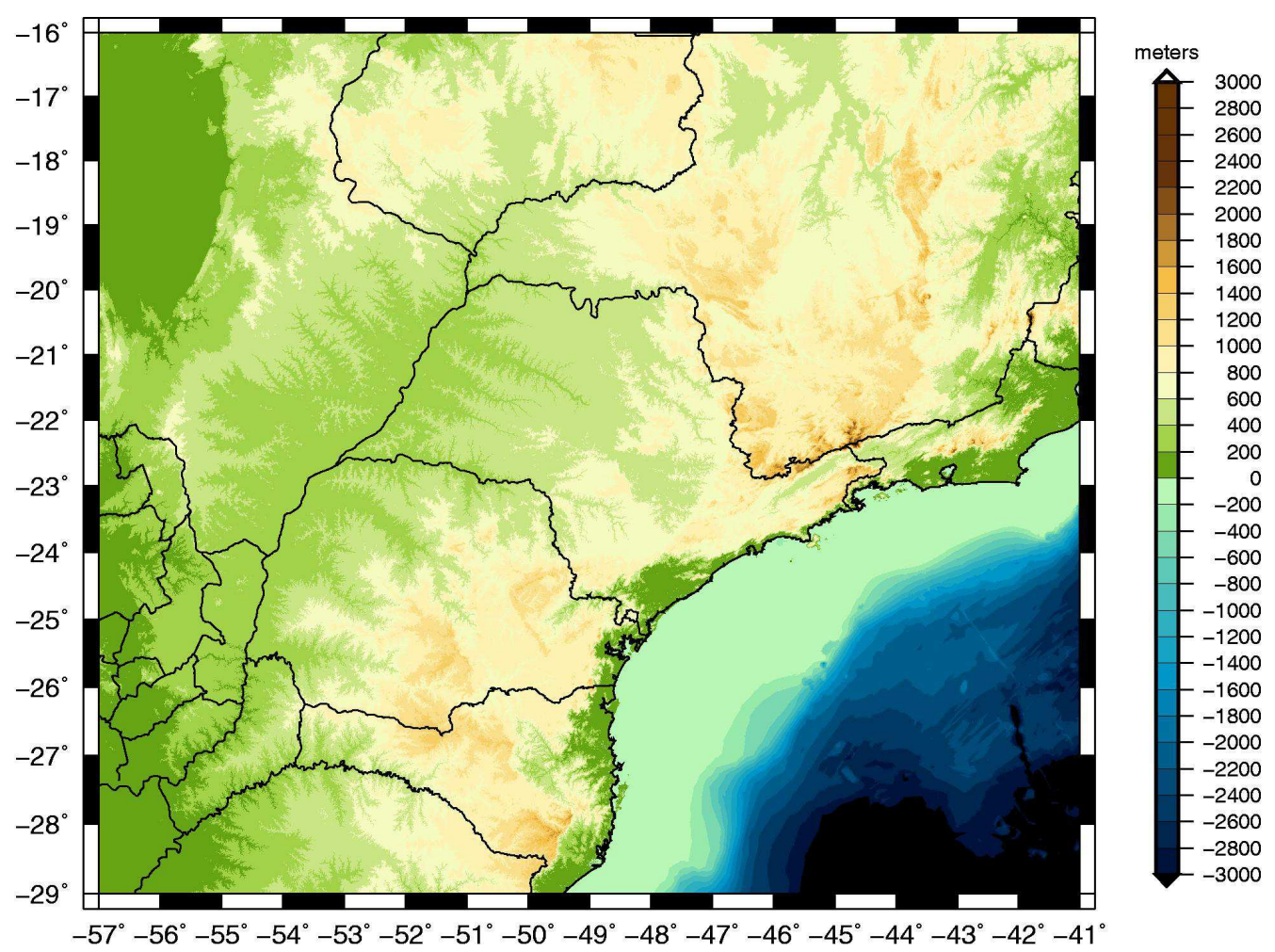

Figure 5.4 - Digital Terrain and Bathymetric Model.

This model consists of SRTM3 (FARR et al., 2007), but EGM96 (LEMOINE et al., 1998a and 1998b) geoid heights used in the SRTM3 was substituted by EIGENGL04C (FÖRSTE et al., 2006) in order to derive the orthometric height. Here the gaps were substituted by digitising maps and DTM2002 (Digital Terrain Model 2002) topographic model (SALEH; PAVLIS, 2002). DTM2002 combines data from GLOBE (Global Land One-kilometer Base Elevation), version 1.0, constructed by the National Oceanic and Atmospheric Administration (NOAA) and the National Geophysical Data Center (NGDC) (HASTING; DUNBAR, 1999), and ACE (Altimeter Corrected Elevation), from Earth and Planetary Remote Sense Laboratory, University of Montfort, UK. In the ocean the global model DTU10 was used (ANDERSEN, 2010). A comparison (Table 5.2) involving two geopotential model (EGM96 and EIGENGL04C) with 706 GPS/leveling data in Brazil was carried out to evaluate the DTM.

Table 5.2 - Statistics analysis of the geopotential models used in DTM.

$$
\text { EGM96 (m) EIGEN-GL04C (m) }
$$

$\begin{array}{lll}\text { Mean } & -0.39 & -0.32 \\ \text { RMS } & 0.88 & 0.76\end{array}$




\subsection{GPS Leveling}

In this thesis 363 GPS/leveling stations (Figure 5.5) was selected. The spirit leveling was carried out by the Brazilian surveying institute IBGE and the former IGG (Instituto Geográfico e Geológico), currently IGC (Instituto Geográfico e Cartográfico). The orthometric heights are referred to a local height datum (Imbituba tide gauge) and the ellipsoidal heights to WGS84 ellipsoid.

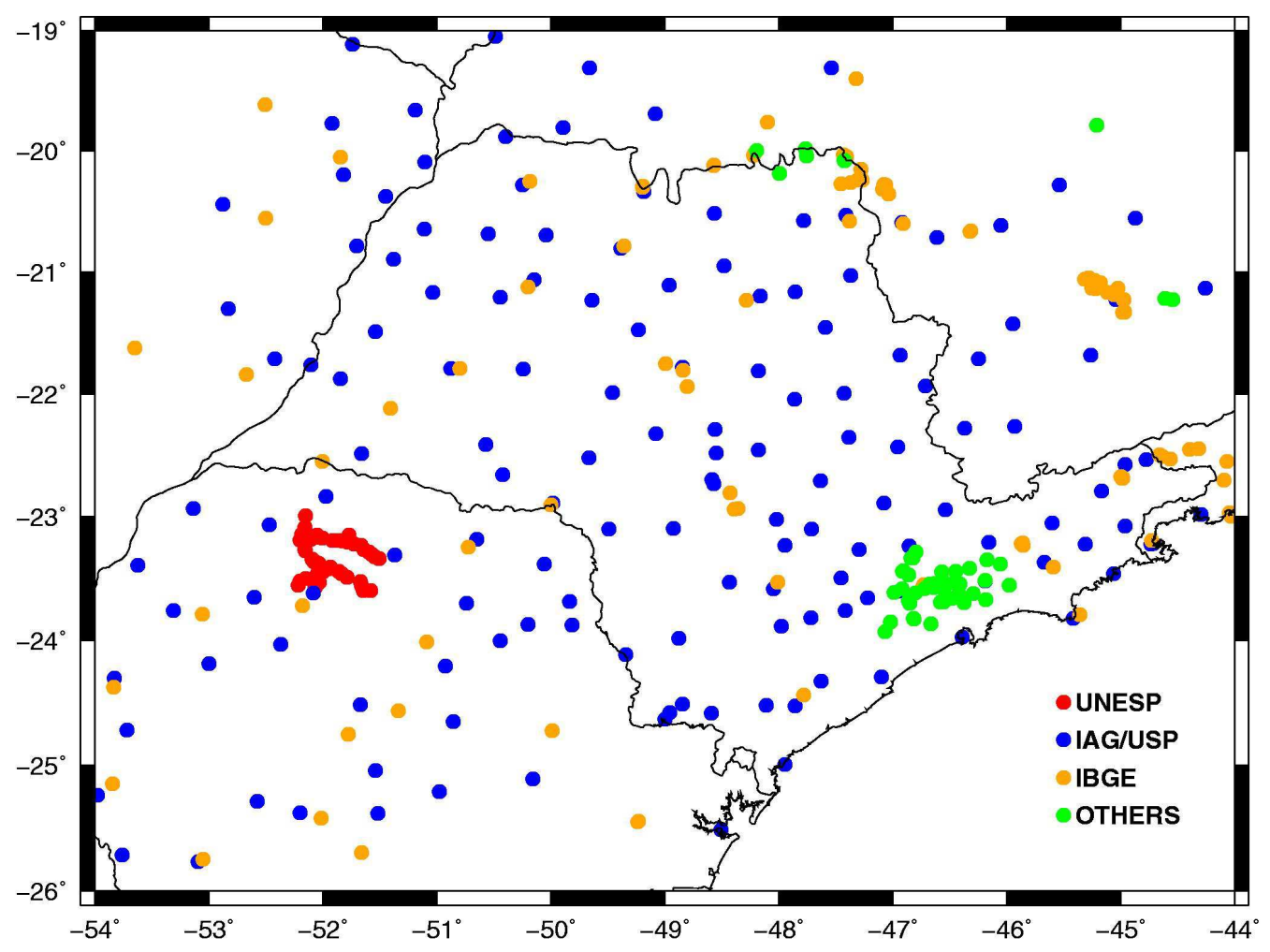

Figure 5.5 - GPS/leveling distribution.

Out of this total, 154 stations belong to IAG. The ellipsoidal height accuracy is about 0.06m (SÁ; VIEIRA, 2006) and it is not possible to define the orthometric height accuracy, since the network was not adjusted. Furthermore, 113 stations were provided by IBGE and they are included in the latest Brazilian altimetric adjustment (IBGE, 2011). Figure 5.6 illustrates the standard deviations of both quantities. In terms of orthometric height, the standard deviations vary from 0.04 and $0.09 \mathrm{~m}$, while the accuracy of the ellipsoidal heights ranges from few millimeters to $0.12 \mathrm{~m}$. 


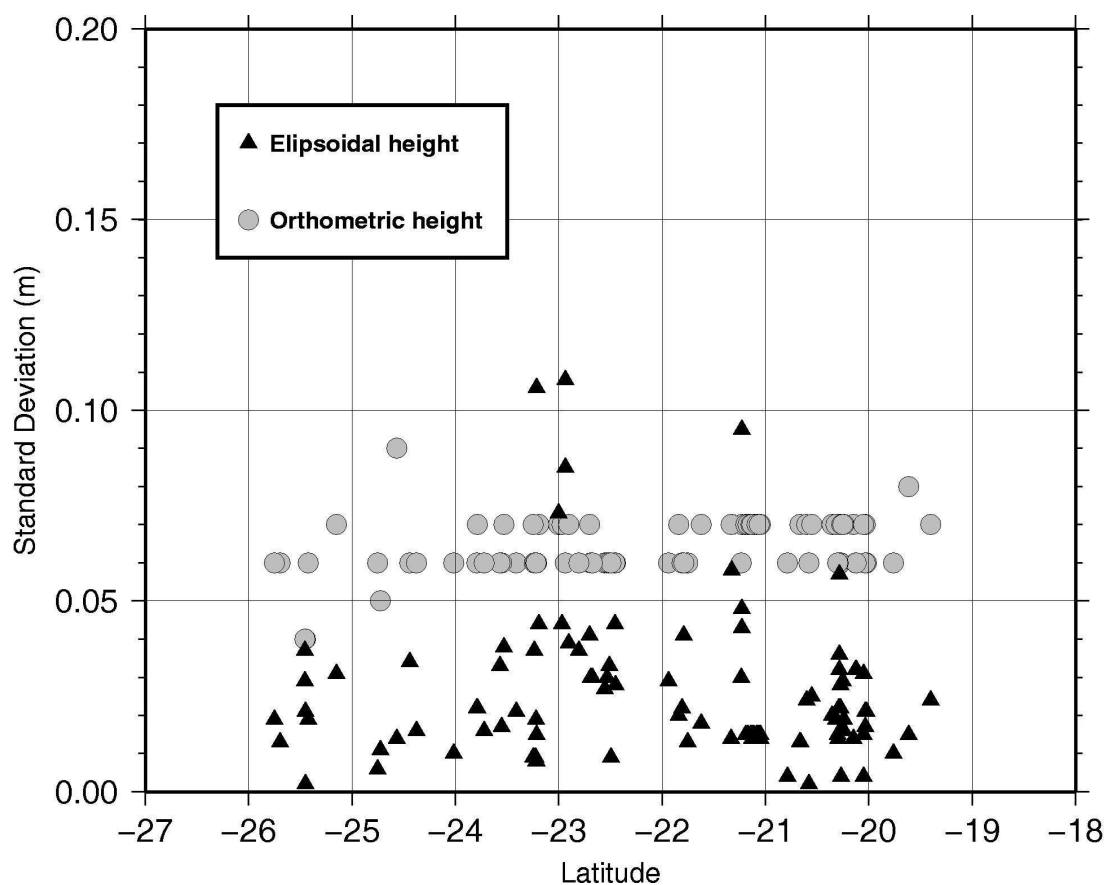

Figure 5.6 - IBGE GPS/leveling standard deviation. 


\section{ESTIMATION PROCEDURES AND RESULTS}

This chapter focuses in the procedures and results in the computation of the geoid model in the State of São Paulo using FFT and LSC techniques. The comparisons involving the two methodologies are carried out. Moreover, it will be discussed the results in terms of absolute and relative comparisons with GPS/leveling. Also, it will be presented the results regarding the evaluation of the GOCE-based model in terms of geoid heights and gravity disturbances.

\subsection{Geoid Model Computed by FFT}

The schedule to determine the geoid model using FFT (Figure 6.1) can be described in 5 steps (BLITZKOW, et al. 2008):

1. Calculation of point free air gravity anomalies through terrestrial gravimetric data (coordinates, orthometric height and gravity acceleration);

2. Calculation of complete Bouguer anomalies in order to derive mean free air gravity anomalies. The 5' x 5' grid of these anomalies was computed from point gravity data. Over the ocean, DTU10 was used.

3. Calculation of Helmert gravity anomalies referred to the surface of the Earth, which are obtained from the mean free air anomaly by adding Direct Topographical Effect (DTE), Direct Atmospheric Effect (DAE) and Secondary Indirect Topographical Effect (SITE) (ELLMAN; VANIČEK, 2007);

4. Stokes' integration with the use of the spectral decomposition to calculate the co-geoid. The modified Stokes' kernel was computed according to Featherstone (2003);

5. Primary Indirect Topographical Effect (PITE) was added to co-geoid heights to obtain geoid heights (MARTINEC; VANIČEK, 1994) and (MARTINEC, 1998). 


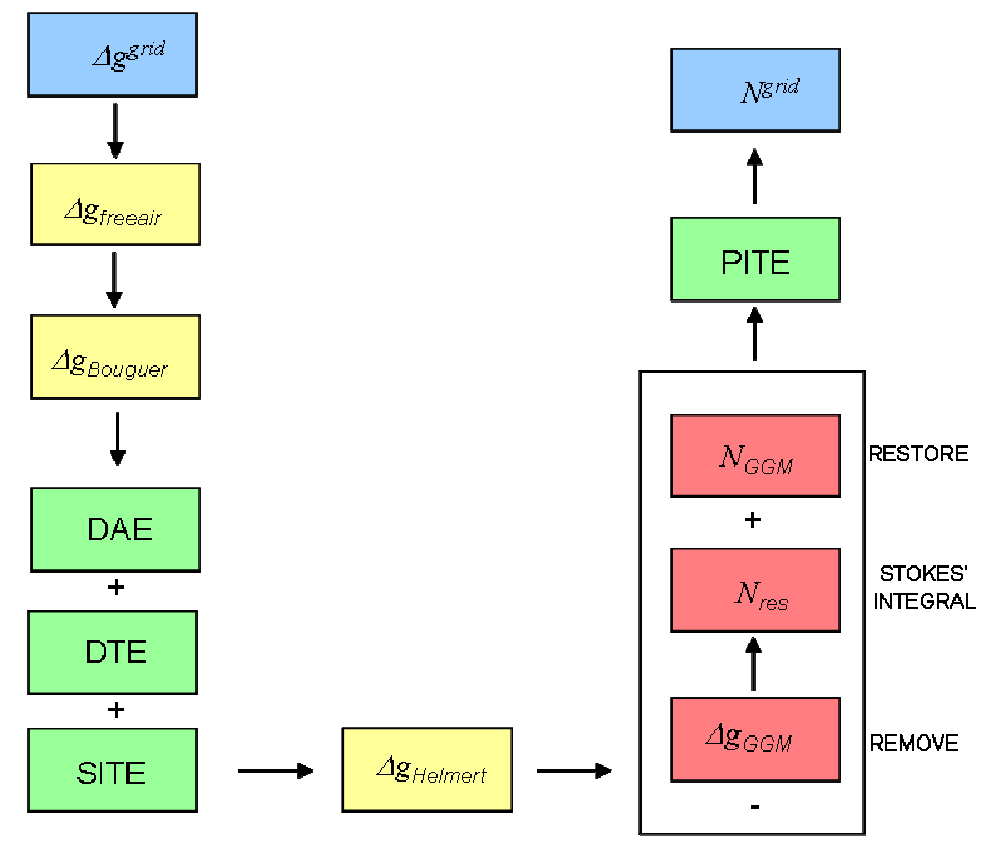

Figure 6.1 - FFT scheme computation.

The SHGEO (Stokes-Helmert GEOid software) is a scientific software for a precise geoid model calculation based on Stokes-Helmert theory for the determination of the gravimetric geoid (ELLMAN, 2005a; 2005b). It was developed in the University of New Brunswick, Canada. In this thesis the software was used to compute Helmert gravity anomalies.

Bouguer anomaly was computed in a 5' resolution grid. The SAM3s_v2 with 3" resolution digital terrain model was used (MATOS; BLITZKOW, 2008). The spectral decomposition consisted in remove from the gravity mean anomalies the long wavelength component provided by GGMs up to a certain degree and order. Stokes' integral was computed using a value for $\psi_{0}$, then the GGM long wavelength component was restored in the geoid height with the same degree and order. The integral computation was carried out by FFT technique using FFTMOD program (LI; SIDERIS, 1993) with the kernel modification proposed by Featherstone; Evans; Olliver, 1998. This modification (MEISSL, 1971) is achieved by simply subtracting the numerical value of the spherical Stokes' kernel at the truncation radius $S\left(\psi_{0}\right)$, from the original kernel (4.18). Helmert gravity anomaly referred to the Earth's surface is presented in Figure 6.2 


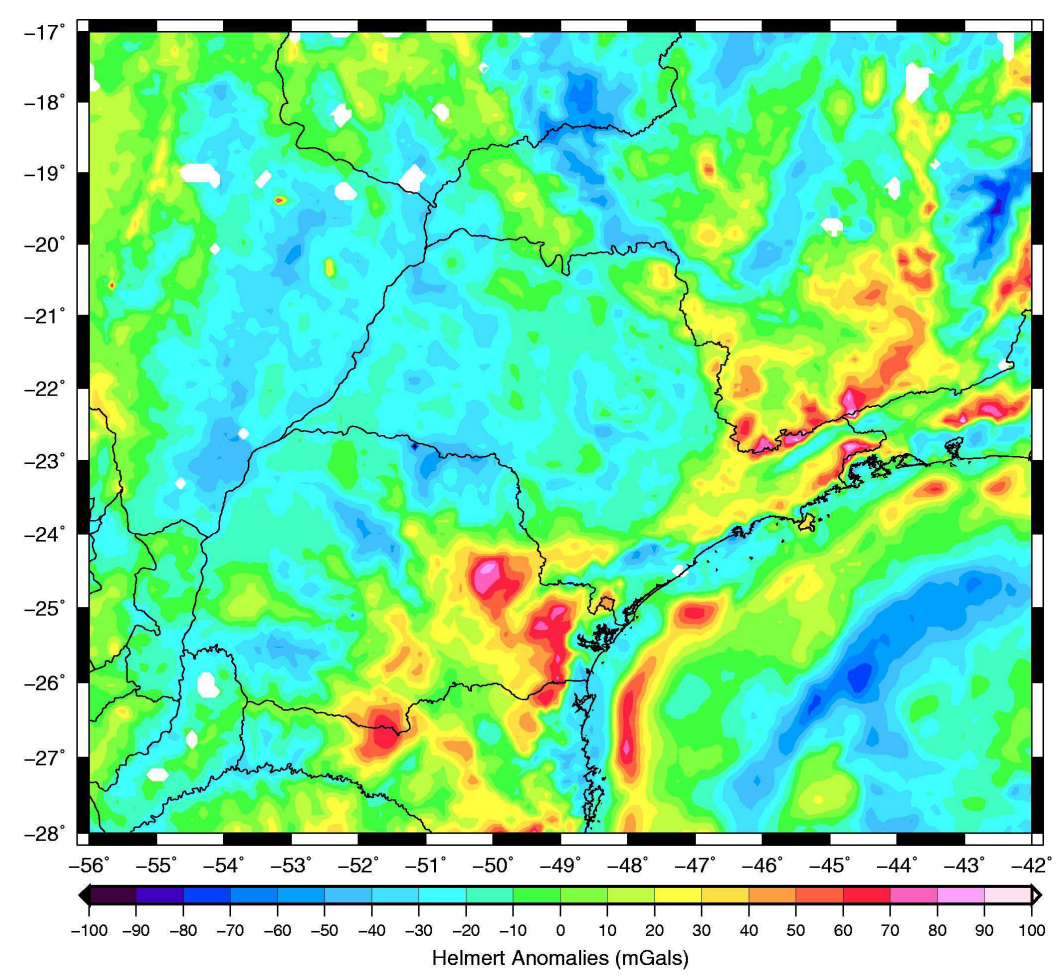

Figure 6.2 - Helmert anomalies (white gaps there is no data).

In the geoid model computation, GGMs were truncated up to degree and order 150, once the long wavelength component provided by the satellites is better defined in this range of frequencies. Also some tests were made for $n=m=360$. Table 6.1 shows the statistic involving Helmert anomalies and the geoid heights using EGM2008 as GGM up to degree and order 150 and 360 as reference field.

Table 6.1 - Helmert anomalies and FFT geoid height statistics.

\begin{tabular}{lrccc}
\hline & \multicolumn{4}{c}{ mGal } \\
\cline { 2 - 5 } & Mean & $\begin{array}{c}\text { Std. } \\
\text { Dev. }\end{array}$ & Max. & Min. \\
\hline Helmert anomalies & -8.81 & 24.04 & 104.06 & -132.80 \\
Helmert anomalies residual EGM2008 (150) & -8.99 & 18.35 & 43.02 & -52.13 \\
Helmert anomalies residual EGM2008 (360) & -8.84 & 21.28 & 70.67 & -70.26 \\
& & meters & \\
\cline { 2 - 5 } & Mean & Std. & Max. & Min. \\
\hline Geoid heights residual EGM2008 (150) & 0.01 & 0.44 & 1.76 & -1.31 \\
Geoid heights residual EGM2008 (360) & 0.00 & 0.14 & 0.92 & -0.68 \\
Geoid heights EGM2008 (150) & -3.22 & 3.64 & 5.32 & -10.75 \\
Geoid heights EGM2008 (360) & -3.77 & 3.15 & 5.61 & -11.31 \\
\hline
\end{tabular}


Figure 6.3 represents the geoid model in the State of São Paulo. EGM2008 up to degree and order 150 was used as reference field.

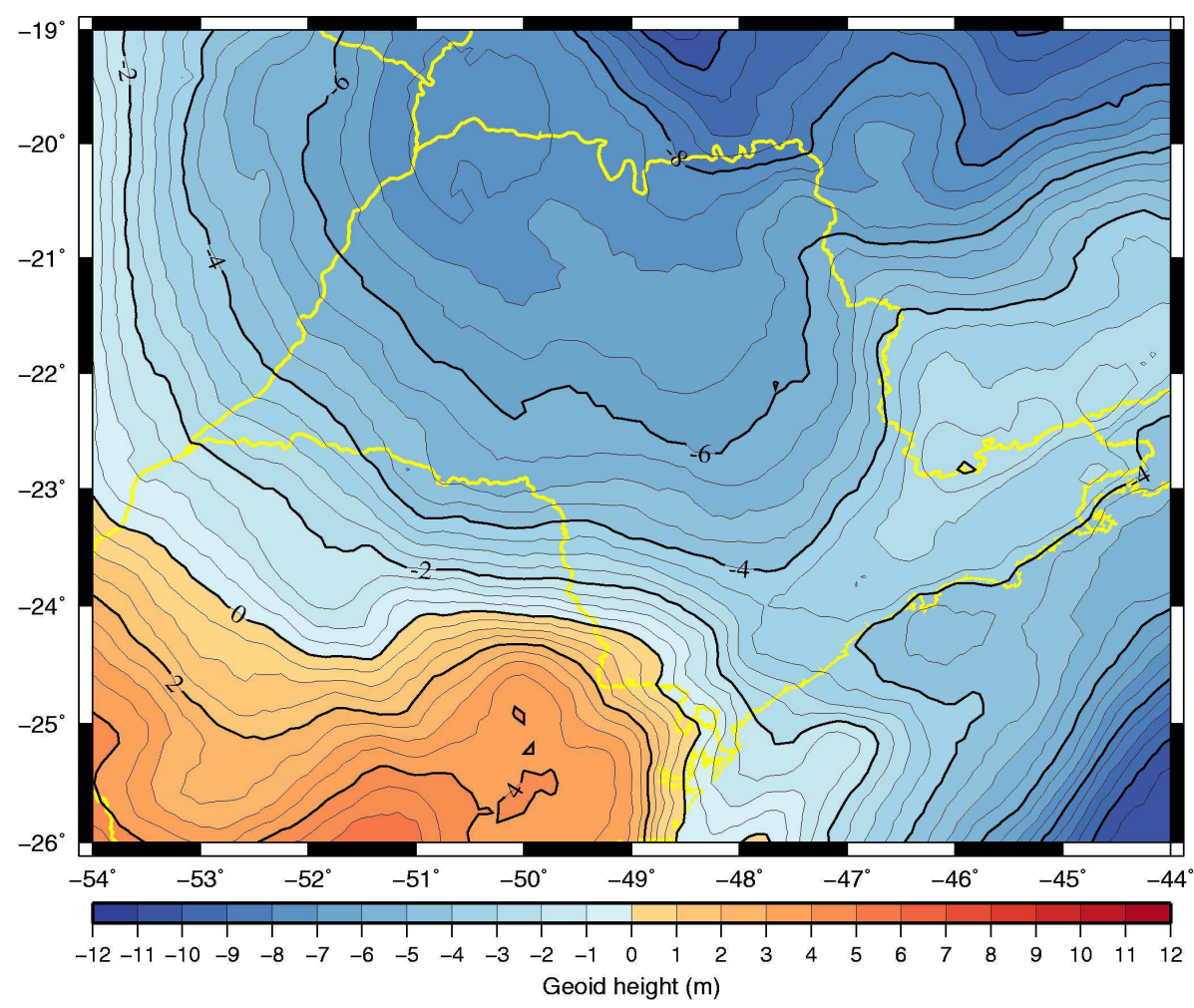

Figure 6.3 - Geoid model by FFT.

\subsection{Geoid Model Computed by LSC}

The methodology to compute the geoid using LSC (Figure 6.4) is described below:

1. Calculation of point free air gravity anomalies through terrestrial gravimetric data (coordinates, orthometric height and gravity acceleration);

2. Use the remove-restore technique to remove the long-wavelength component from the geopotential model and the residual terrain correction. Over the ocean, a refined DTM/bathymetry model (DTU10) is set up in order to estimate the RTC effect. This has been accomplished by merging the SRTM DTM with the available NOAA bathymetry of the Atlantic Ocean in the computation area;

3. Residual gravity anomaly interpolation in a 5' grid;

4. Computation of the empirical and model covariance functions; 
5. Computation applying the Fast Collocation method (BOTONI; BARZAGHI, 1993);

6. Restore the long-wavelength component and the residual terrain correction to obtain the geoid height.

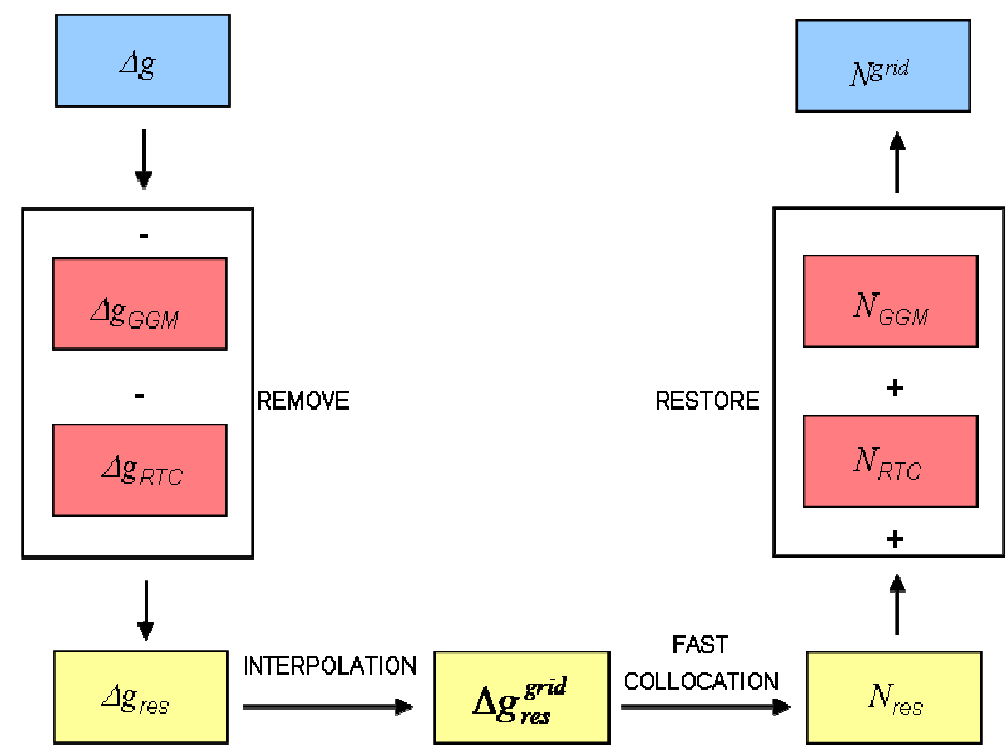

Figure 6.4 - LSC scheme computation.

The GRAVSOFT (Geodetic Gravity Field Modelling Programs) package is a set of programs for basic operations of Physical Geodesy. The software was developed under the supervision of the National Space Institute and the University of Copenhagen, both in Denmark (FORSBERG; TSCHERNING, 2008). In this thesis, it was used to compute the terrain reductions and the covariance functions (empirical and model).

The residual gravity anomaly was obtained subtracting from the gravity anomalies the long wavelength component provided by GGM and the residual terrain correction provided by DTM. The gravimetric residual terrain correction is the topographic irregularities effect with respect to the mean bouguer. In order to apply Fast Collocation, it was needed to produce a grid of residual values from sparse gravity points. The grid was computed for a 5' resolution using the weighted mean interpolation method.

The empirical covariance function was computed on a spherical surface by means of products-sums samples of scalar values or of the longitudinal and transversal components of vector quantities (FORSBERG; TSCHERNING, 2008). From the 
empirical covariance is possible to argue some important characteristics, where three parameters are essential. The variance $C_{0}$ is the value of the covariance function $C_{\psi}$ for $\psi=0$. The correlation length $\xi$ is the value of the argument for which $C_{0}$ is the half of its value at $\psi=0$. Finally, the curvature parameter $\chi$ is a quantity related to the curvature function when $\psi=0$.

Figure 6.5 shows the empirical covariance of the residual gravity anomalies using EGM2008 up to degree and order 150. The function was fitted by Tscherning-Rapp model and computed by COVFIT program from GRAVSOFT package. The Bjerhammar radius used was $6,300 \mathrm{~km}$ and the scalar factor 0.2 . The optimal radius selected was 0.80 .

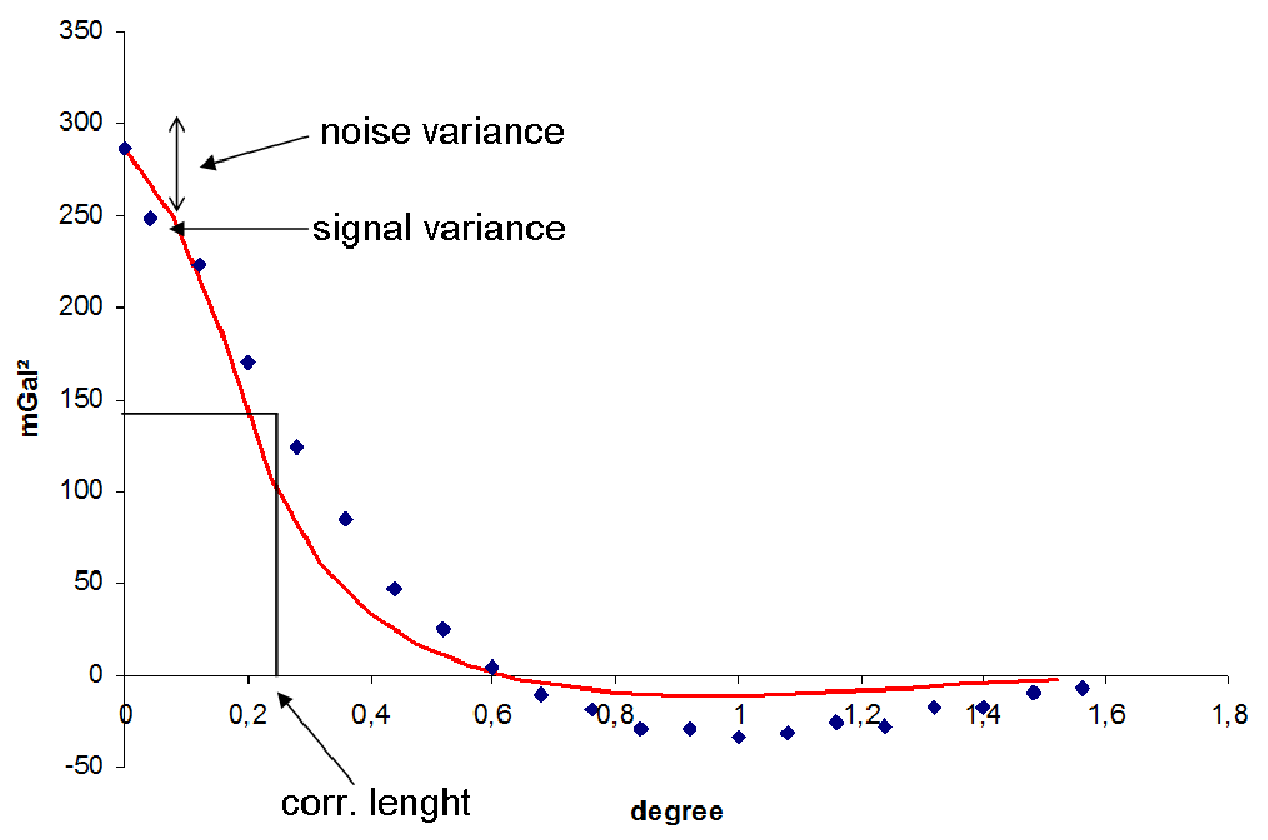

Figure 6.5 - Empirical covariance (blue) and fitted model (red) using EGM2008 up to n=m 150.

The data distribution is an important factor to fit the covariance functions. The lack of data may lead to poor modelling. The overabundance or the excessive amount of data overwhelms the system with excessive use of computational memory. To understand the covariance functions behavior, gravity data were divided in 5 blocks by $3^{\circ}$ in latitude and longitude (Figure 6.6). These blocks were named by letters A, B, C, D and E. 


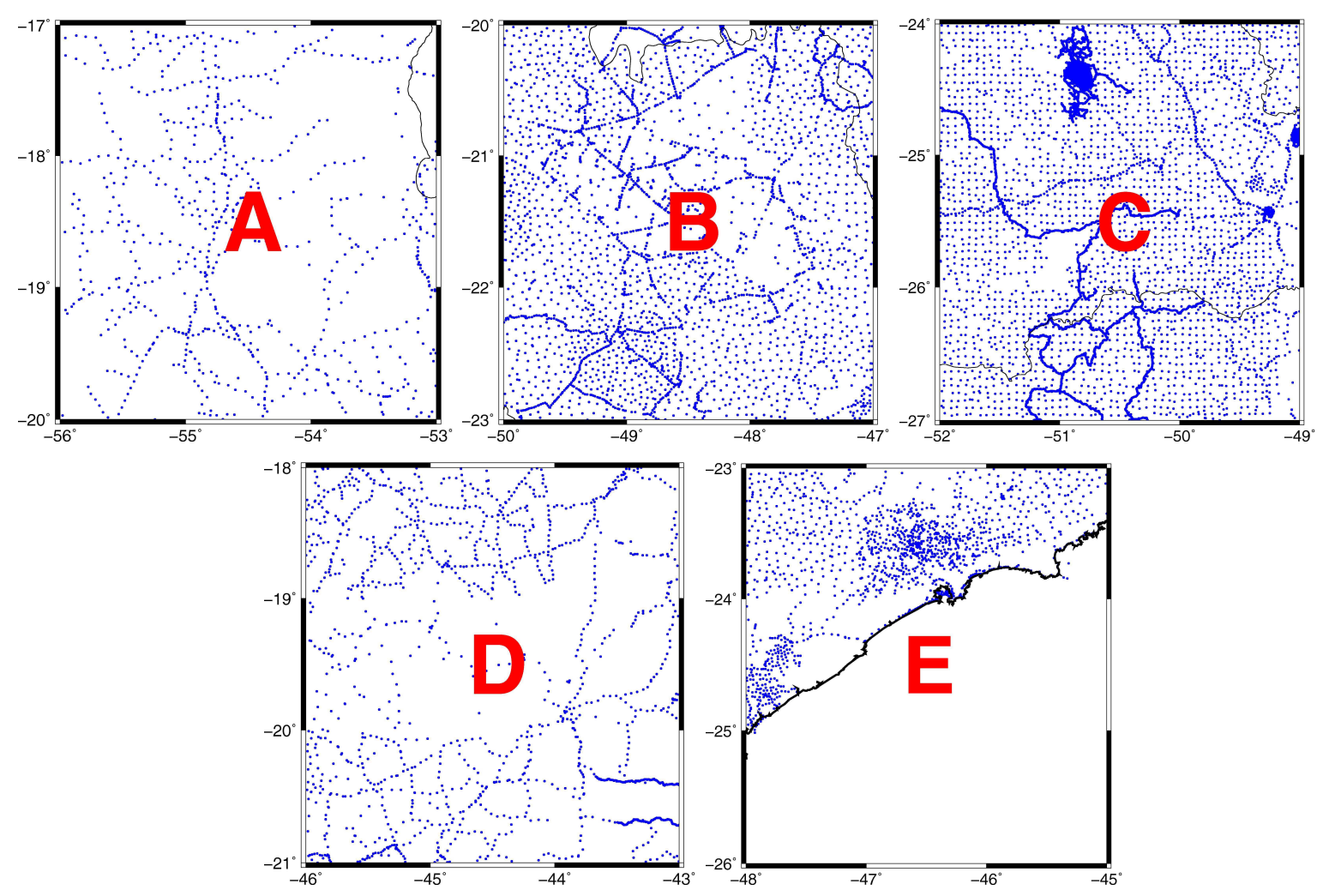

Figure 6.6 - Area blocks.

The covariance function and consequently the parameters are influenced by data distribution, as well as the topography. Hence, for the study region would not be convenient to choose a single function, because the estimated covariances vary significantly from one region to another. The main difficult of using data with irregular distribution is to verify the isotropy and homogeneity. The empirical covariance functions were computed using EMPCOV program and the obtained values were used to compute the fitted covariance functions by applying COVFIT program. Table 6.2 shows the estimated values. The Bjerhammar radius is the most affected parameter in the covariance functions adjustment.

The topography can influence the modelling of covariance functions, since the gravity anomalies are highly correlated with the topography. In this sense, it is expected that in flat areas the variation is smaller than in places where the topography is more rugged. Blocks $A$ and $B$ presented the smallest variances in relation to other blocks; in these regions the topography is flatter than the other regions. 
Table 6.2 - Empirical and fitted variance values for the blocks.

\begin{tabular}{ccc}
\hline Block & $\begin{array}{c}\text { Empirical } \\
\text { Variance } \\
\left(\text { mGal }^{2}\right)\end{array}$ & $\begin{array}{c}\text { Fitted } \\
\text { Variance } \\
\left.\text { (mGal }^{2}\right)\end{array}$ \\
\hline$A$ & 127.46 & 127.26 \\
$B$ & 117.46 & 117.32 \\
C & 428.08 & 427.62 \\
$D$ & 227.09 & 226.91 \\
E & 390.62 & 390.13 \\
\hline
\end{tabular}

The Fast Collocation technique was applied to obtain the residual geoid height $N_{\text {res }}$. The restore procedure was used by introducing the long wavelength component from GGM and the residual terrain correction. Table 6.3 shows the statistic involving geoid height residuals and geoid heights using EGM2008 up to degree and order 150 and 360 , as reference field.

Table 6.3 - LSC geoid height statistics.

\begin{tabular}{lcccc}
\hline & \multicolumn{4}{c}{ meters } \\
\cline { 2 - 5 } & Mean & $\begin{array}{c}\text { Std. } \\
\text { Dev. }\end{array}$ & Max. & Min. \\
\hline Geoid height residuals EGM2008 (150) & 0.01 & 0.35 & 1.58 & -1.39 \\
Geoid height residuals EGM2008 (360) & 0.00 & 0.08 & 0.66 & -0.45 \\
Geoid heights EGM2008 (150) & -3.79 & 3.62 & 5.81 & -11.49 \\
Geoid heights EGM2008 (360) & -3.82 & 3.58 & 5.51 & -11.13 \\
\hline
\end{tabular}

Figure 6.7 represents the geoid model in the State of São Paulo computed by Least Squares Collocation. Again, EGM2008 up to degree and order 150 was used in the spectral decomposition to represent the long wavelength component. 


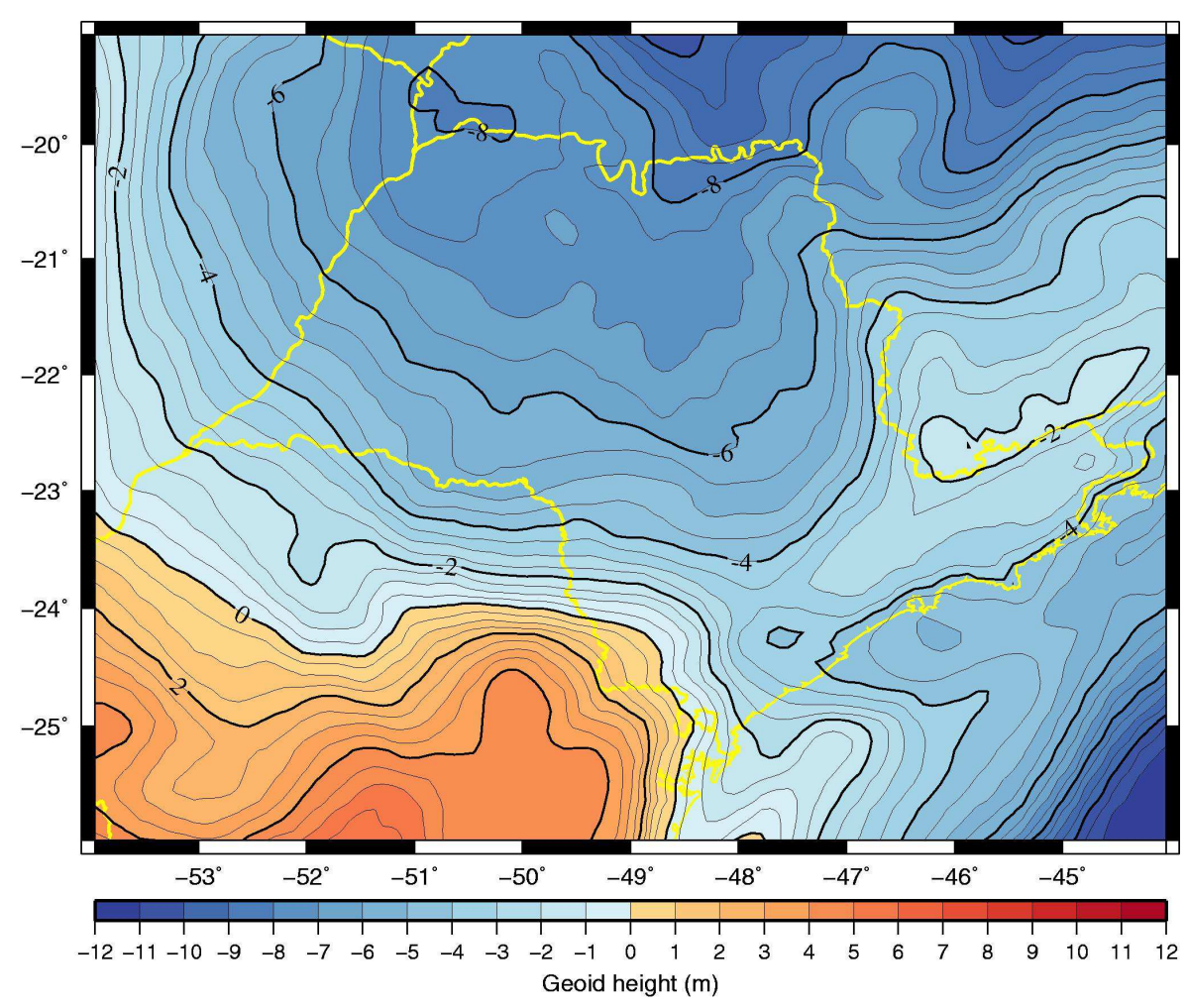

Figure 6.7 - Geoid model by LSC.

\subsection{Geoid Model Comparisons}

Besides EGM2008, presented in figures and tables above, the geoid model was computed using GOCE-based models (DIR_R3 and TIM_R3), GOCO03S and EIGEN-6C in terms of long wavelength component. These geopotencial models were chosen because they are the most recently available models. In the first comparison the geoid height residual difference was analyzed. In the second attempt, the differences between the geoid height provided by the geoid model and the geoid height obtained from GPS observations on Bench Marks of spirit leveling network were evaluated. This evaluation was undertaken in absolute way, while the third comparison was performed in relative way. Also a comparison involving only stations in the mountain area was performed in order to verify FFT and LSC behavior in this region.

\subsubsection{Geoid height residual comparisons}


The geoid height residual was computed by the difference of FFT and LSC residual. This evaluation pretended to verify how is the compatibility of both methodologies in terms of short wavelength component. Figure 6.8 presents the differences.
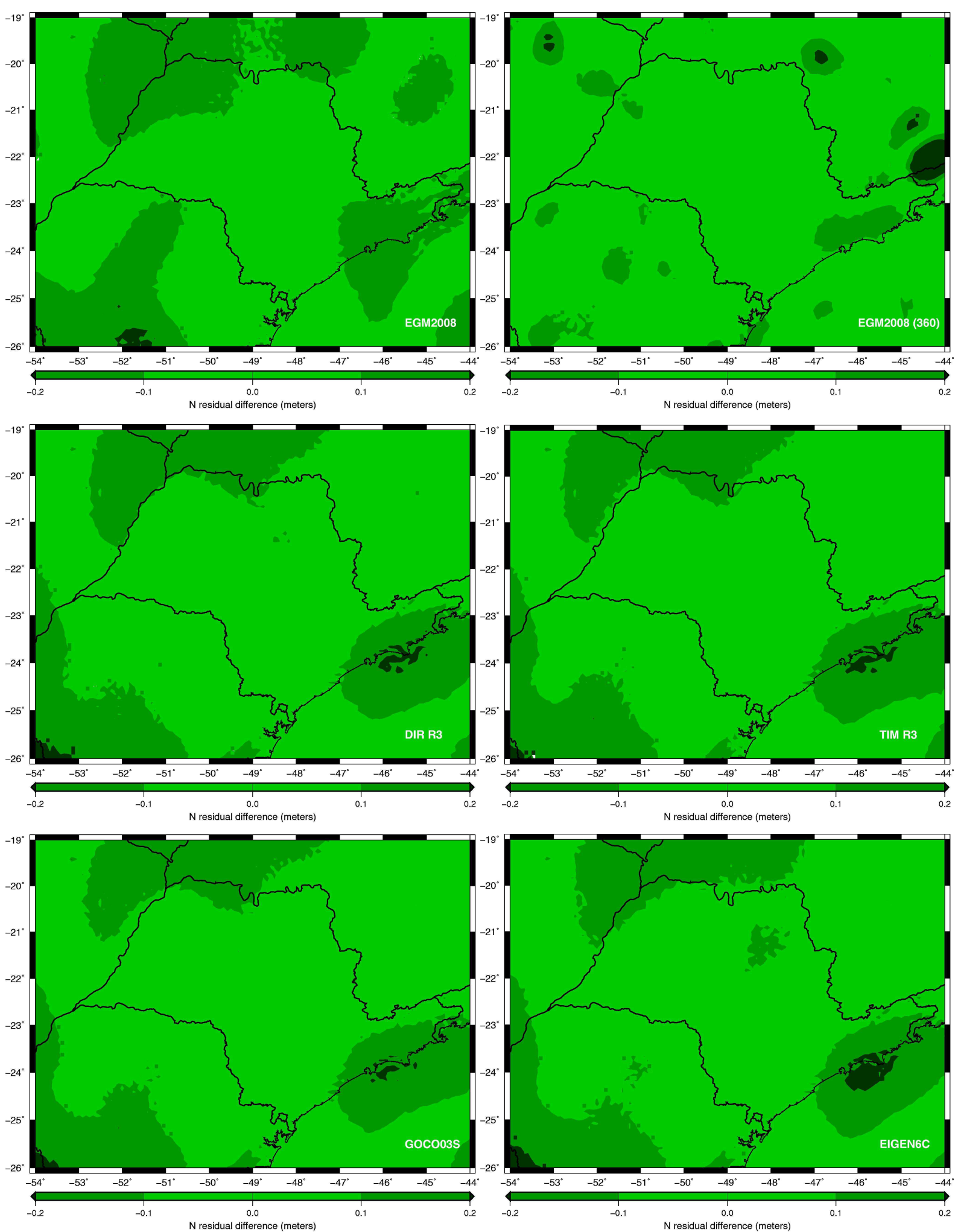

Figure 6.8 - Geoid height residuals. 
Regarding all models, Figure 6.8 shows differences from -0.10 to $0.10 \mathrm{~m}$ in most part of the State of São Paulo. The model computed by EGM2008 ( $n=m=360)$ presented differences in the range from -0.20 to $0.20 \mathrm{~m}$. The reason can be explained by the fact that there are no data in dark green areas and also close to the coast. The geoid models based on GOCE data also presented a small area, close to the coast, with results in the range from -0.20 to $0.20 \mathrm{~m}$. Table 6.4 shows the geoid height residual statistics, where the results for degree and order 150 in terms of mean and RMS difference are the same.

Table 6.4-Geoid height residual statistics.

\begin{tabular}{lcccc}
\hline \multicolumn{1}{c}{ GGMs } & \multicolumn{4}{c}{ meters } \\
\cline { 2 - 5 } & Mean & $\begin{array}{c}\text { RMS } \\
\text { diff. }\end{array}$ & Max. & Min. \\
\hline EGM2008 (150) & 0.02 & 0.08 & 0.22 & -0.25 \\
EGM2008 (360) & 0.01 & 0.06 & 0.49 & -0.26 \\
DIR_R3 (150) & 0.02 & 0.08 & 0.24 & -0.23 \\
TIM_R3 (150) & 0.02 & 0.08 & 0.23 & -0.22 \\
GOCO03S (150) & 0.02 & 0.08 & 0.23 & -0.22 \\
EIGEN-6C (150) & 0.02 & 0.08 & 0.24 & -0.23 \\
\hline
\end{tabular}

\subsubsection{Absolute comparisons}

The absolute comparison allows the analysis on how consistent is the geoid model and the GPS/leveling stations in relation to geoid height. The comparison between these two quantities has been performed in terms of root mean square difference. Figure 6.9 presents the difference of 363 points, on the left the models computed by FFT and on the right by LSC. 

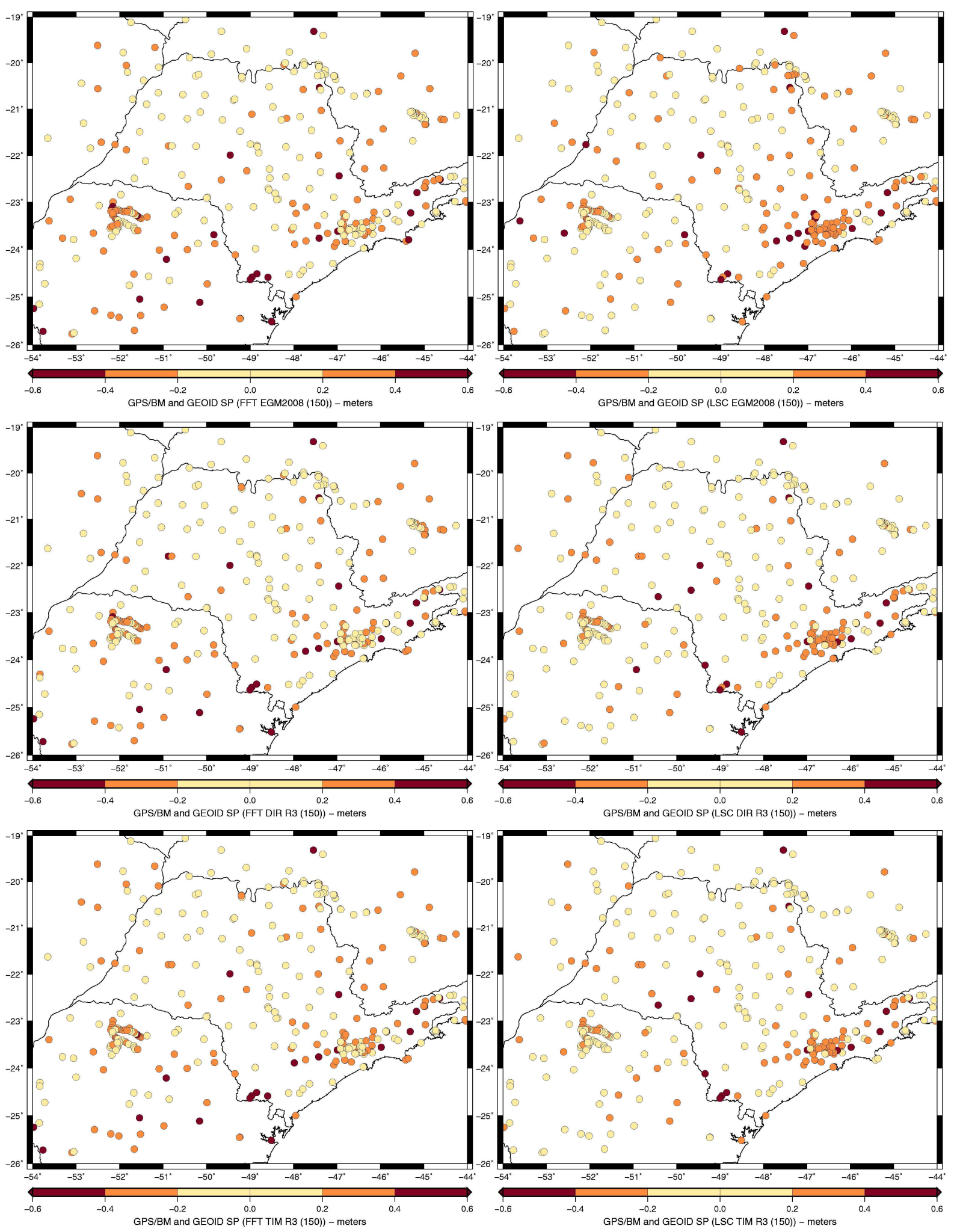


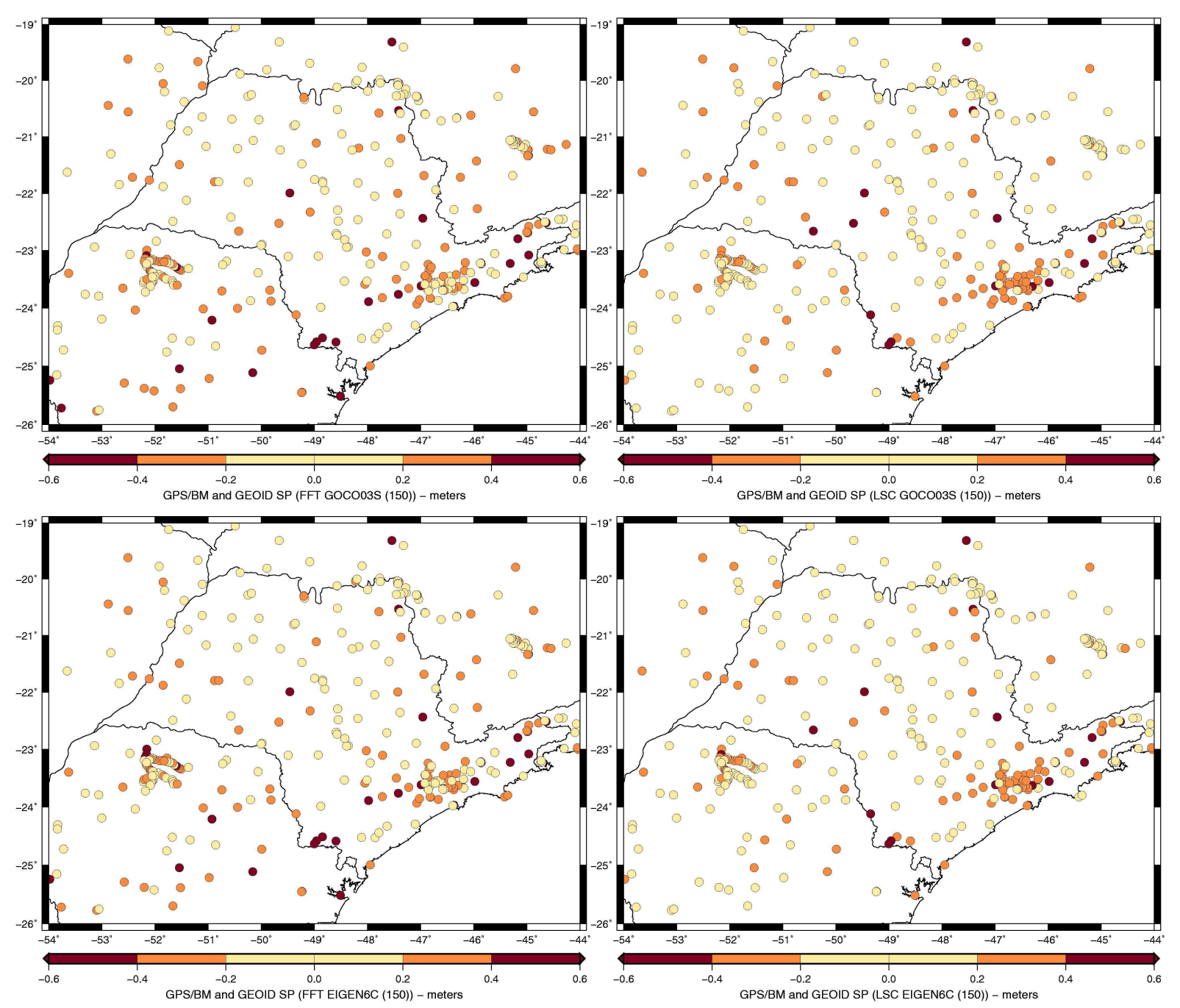

Figure 6.9 - Difference between GPS/leveling and GEOIDSP.

In Figure 6.9, regarding all geoid models, most of the points presented differences in the range from -0.20 to $0.20 \mathrm{~m}$. There is a reasonable quantity of red dark points (above $-0.40 \mathrm{~m}$ and $0.40 \mathrm{~m}$ ) in the region close to the coast. Also, there are some red points scattered in some parts of the model. From 363 stations and considering all models evaluated, $55-65 \%$ of them have differences in the range from -0.20 to 0.20 $\mathrm{m}, 30-40 \%$ between the range -0.21 to $-0.40 \mathrm{~m}$ and 0.21 to $0.40 \mathrm{~m}$ and $5-10 \%$ above $-0.40 \mathrm{~m}$ and $0.40 \mathrm{~m}$. In order to evaluate only the State of São Paulo, some plots were made by the GPS/leveling points interpolation to show the discrepancy throughout the state (Figure 6.10). 

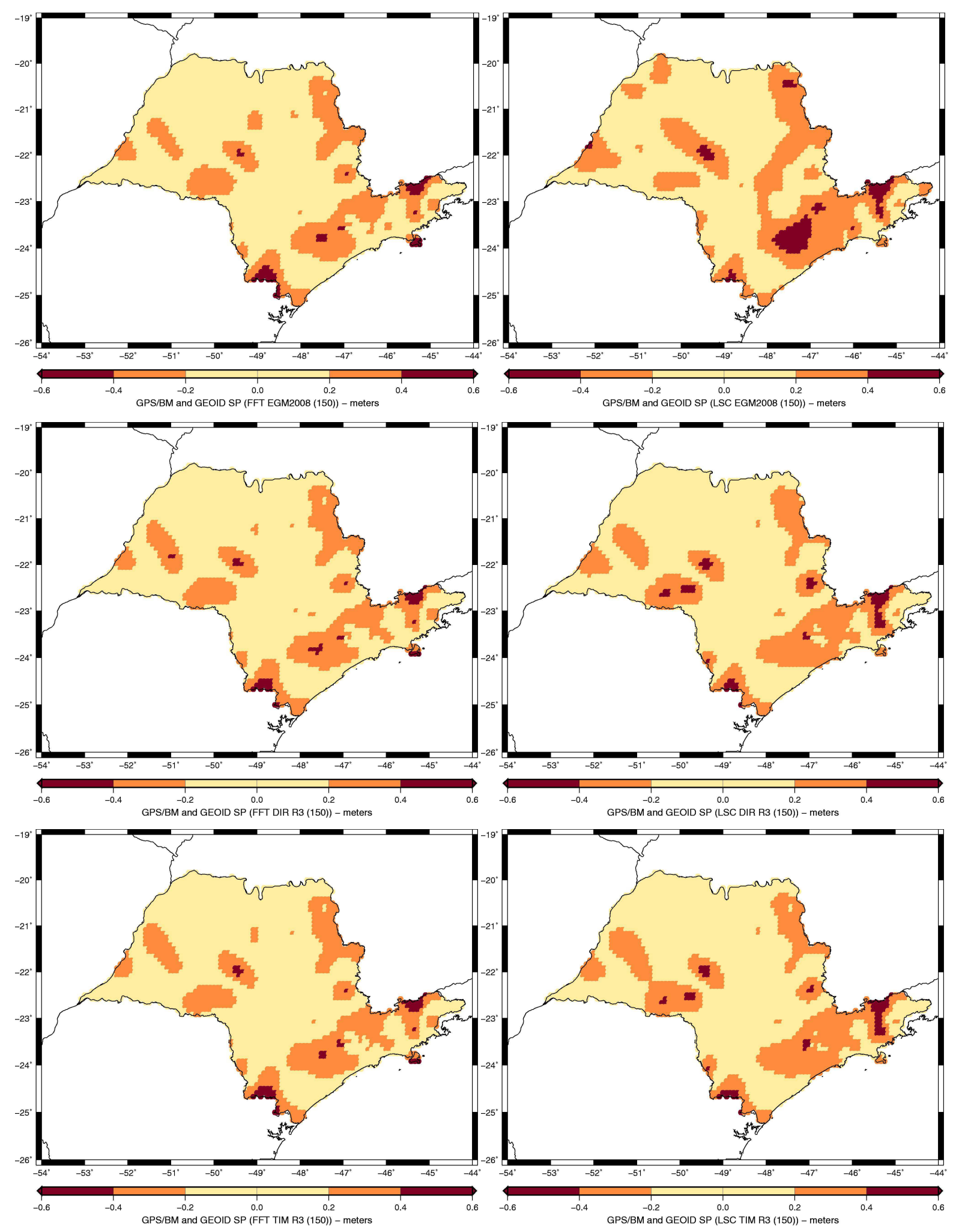


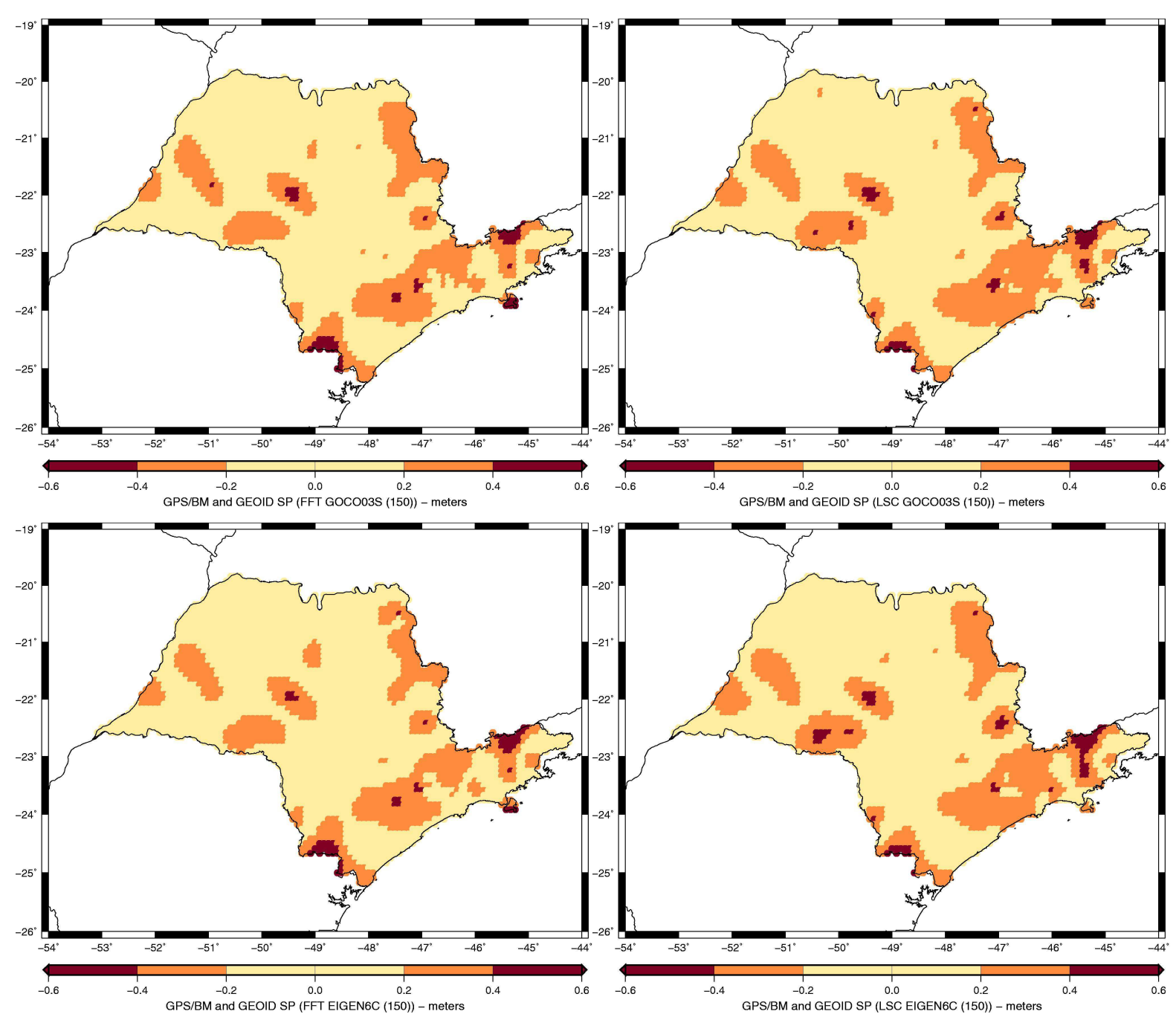

Figure 6.10 - Discrepancy GEOID SP and GPS/leveling in the State of São Paulo

According to Figure 6.10, FFT and LSC have similar behavior for all models except for EGM2008, where LSC presented some red spots larger than FFT, especially in the mountain region. For the geoid models based on GOCE data, the behavior in terms of difference has the same standard. Figure 6.11 presents the histograms of the differences involving all the geoid models computed. The absolute comparison statistics considering all GPS/leveling stations is presented in Table 6.5. 
Histogram GPS/BM and GEOIDSP (FFT EGM2008(150))

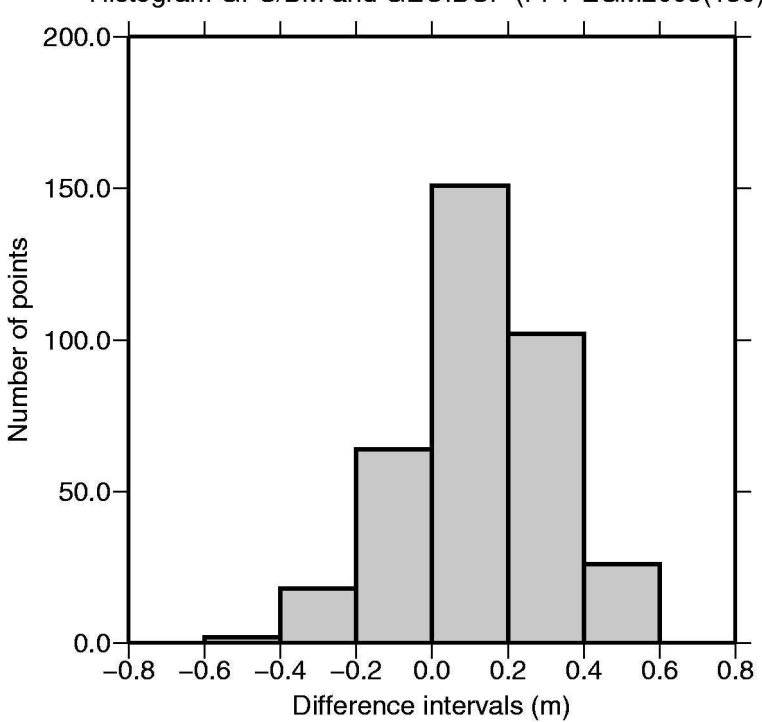

Histogram GPS/BM and GEOIDSP (FFT EGM2008(360))

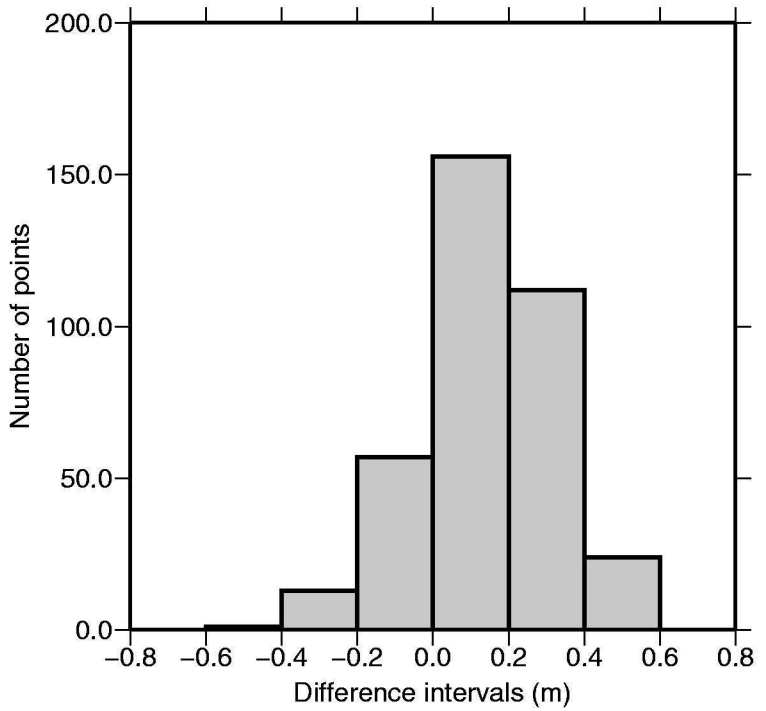

Histogram GPS/BM and GEOIDSP (FFT DIR R3(150))

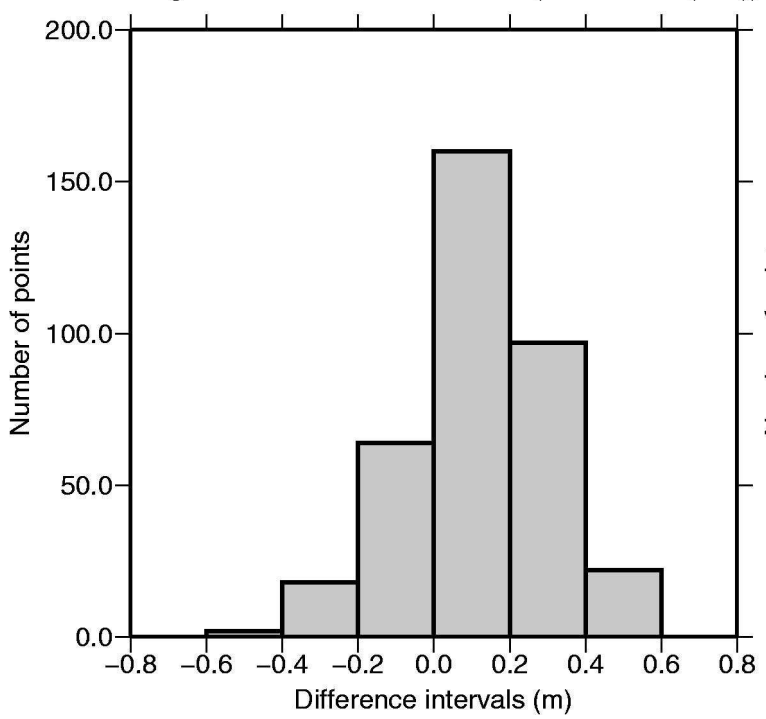

Histogram GPS/BM and GEOIDSP (LSC EGM2008(150))

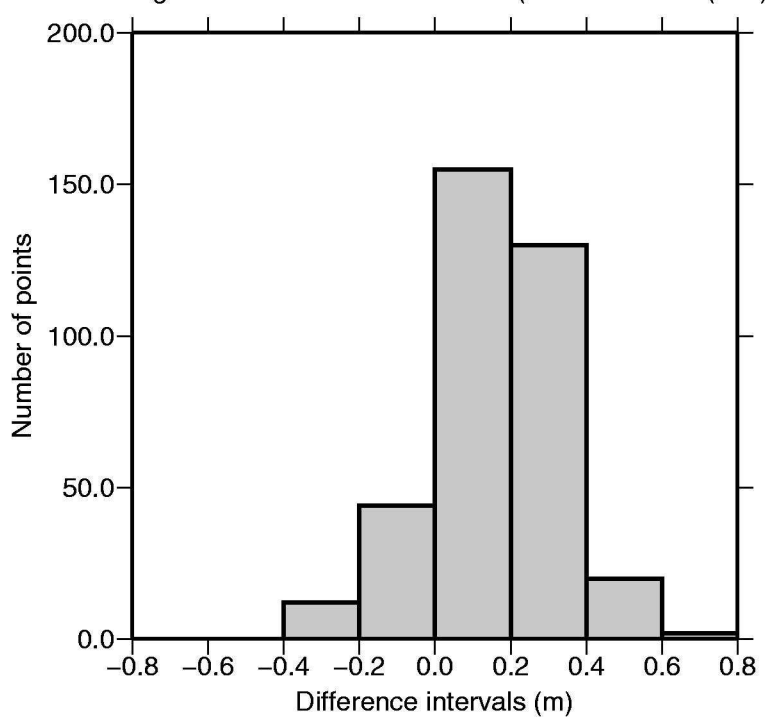

Histogram GPS/BM and GEOIDSP (LSC EGM2008(360))

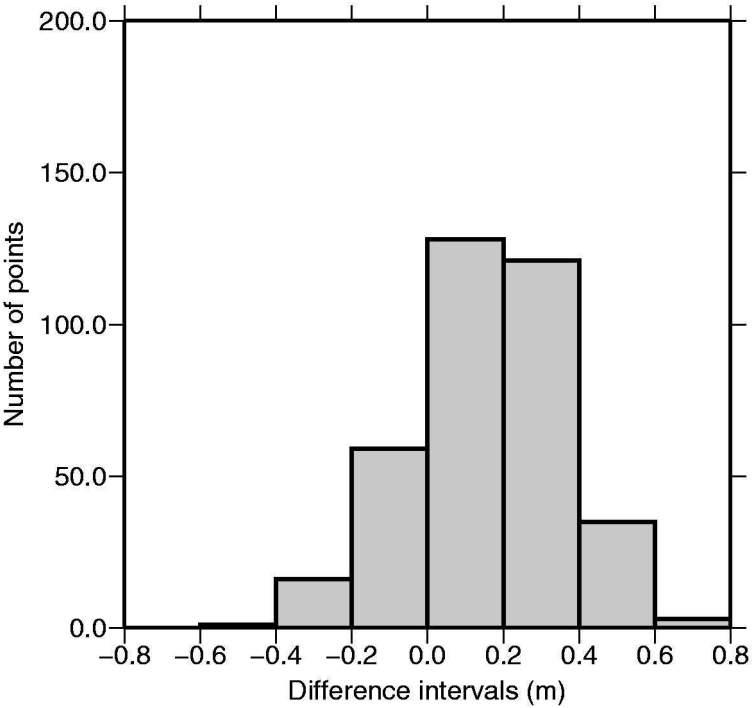

Histogram GPS/BM and GEOIDSP (LSC DIR R3(150))

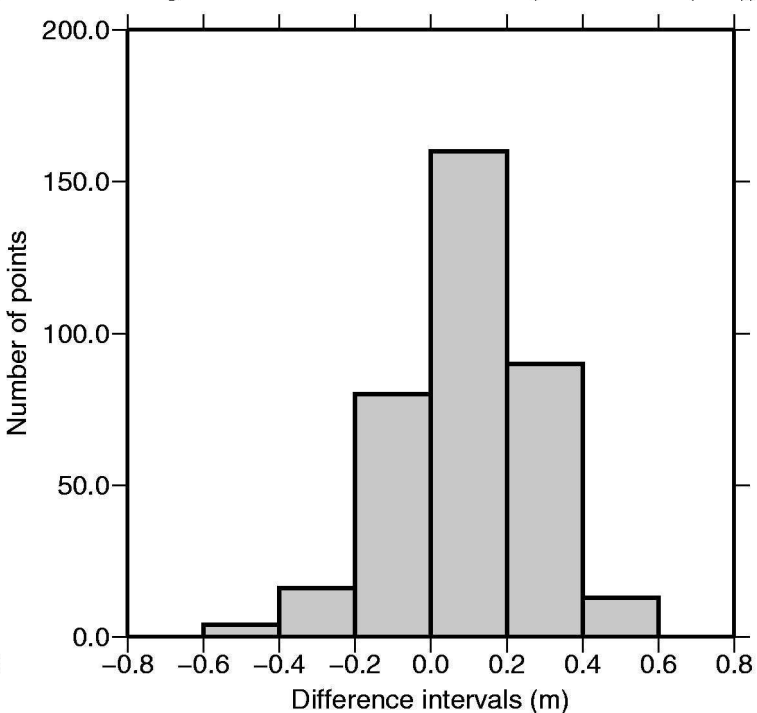



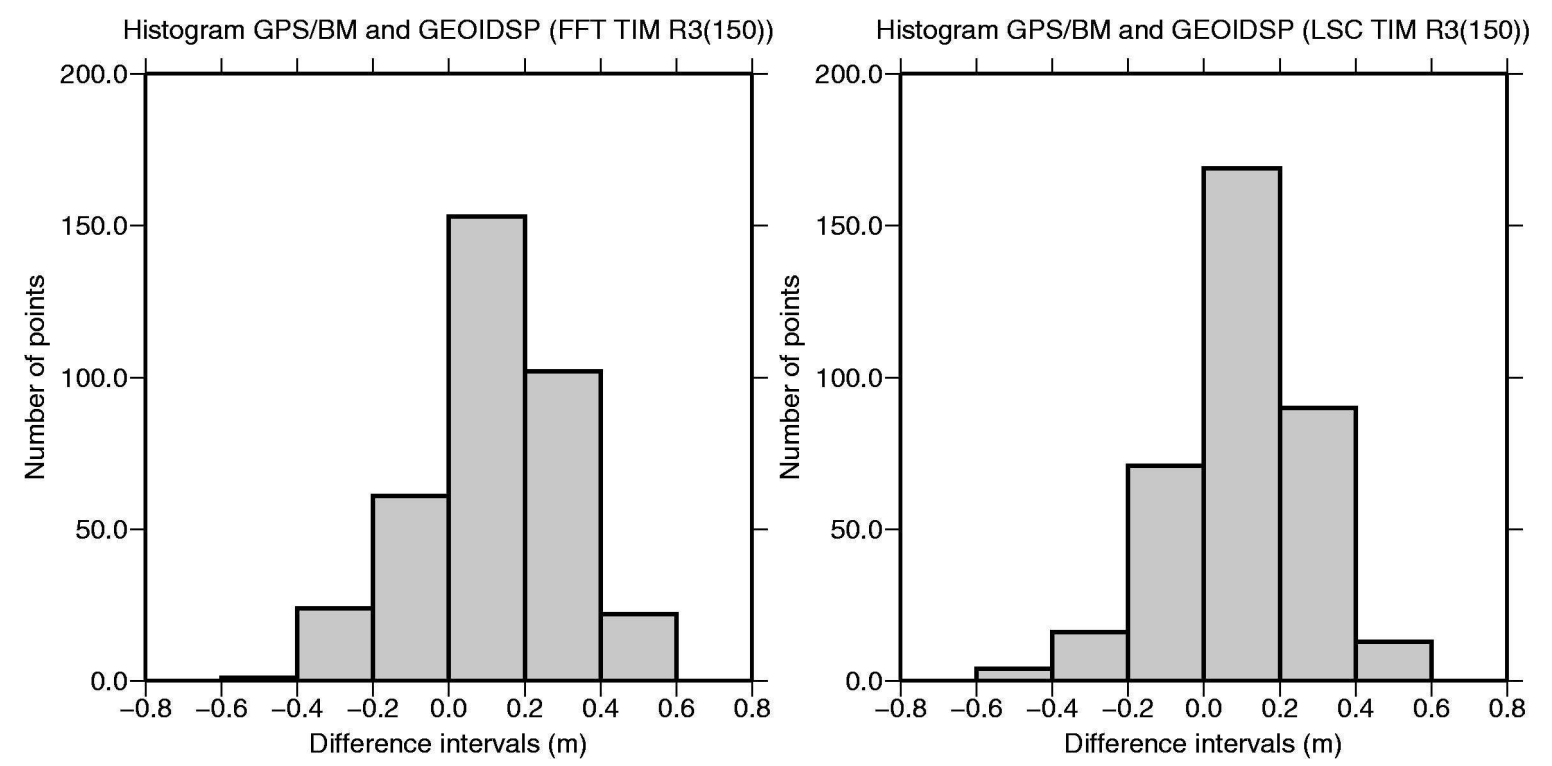

Histogram GPS/BM and GEOIDSP (FFT GOCO03S(150))

Histogram GPS/BM and GEOIDSP (LSC GOCO03S(150))
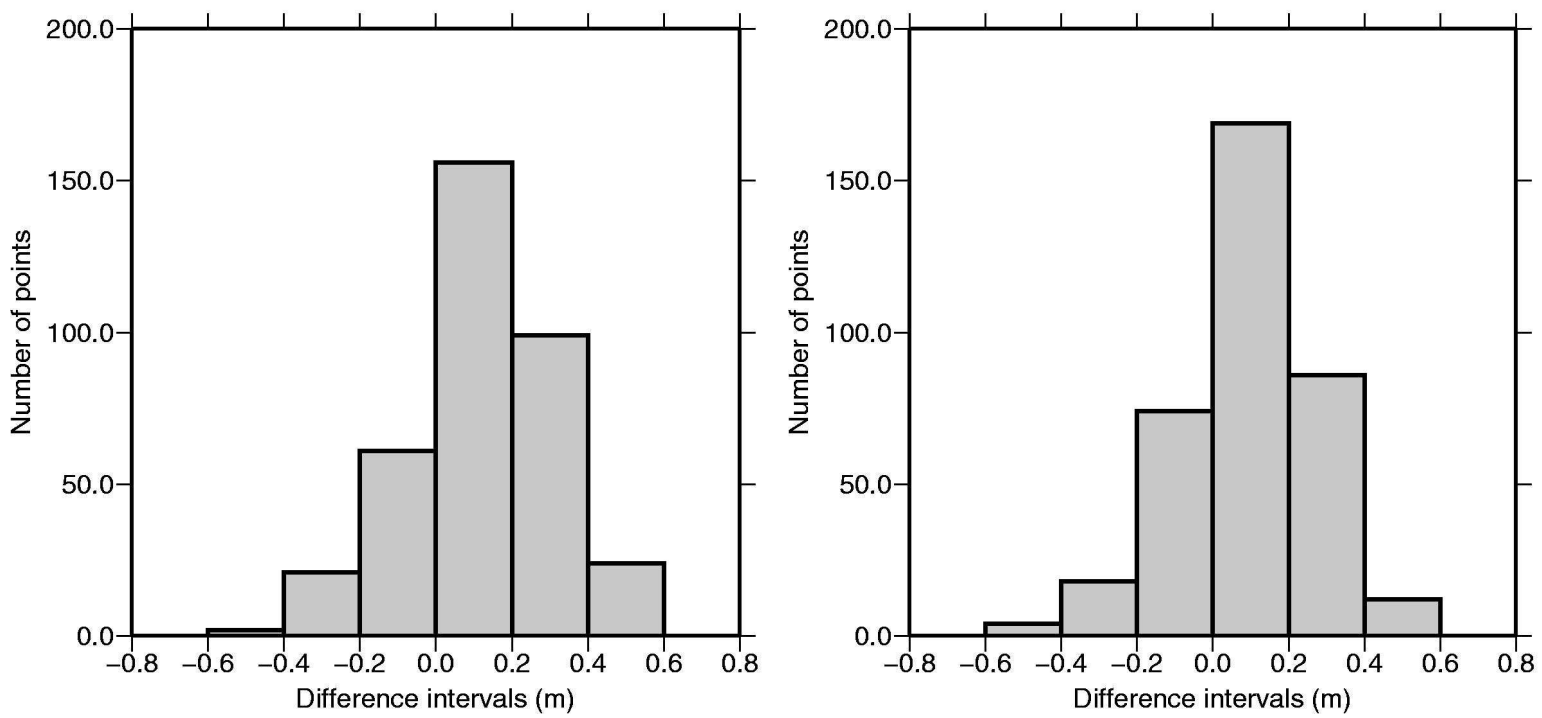

Histogram GPS/BM and GEOIDSP (FFT EIGEN6C(150))

Histogram GPS/BM and GEOIDSP (LSC EIGEN6C(150))
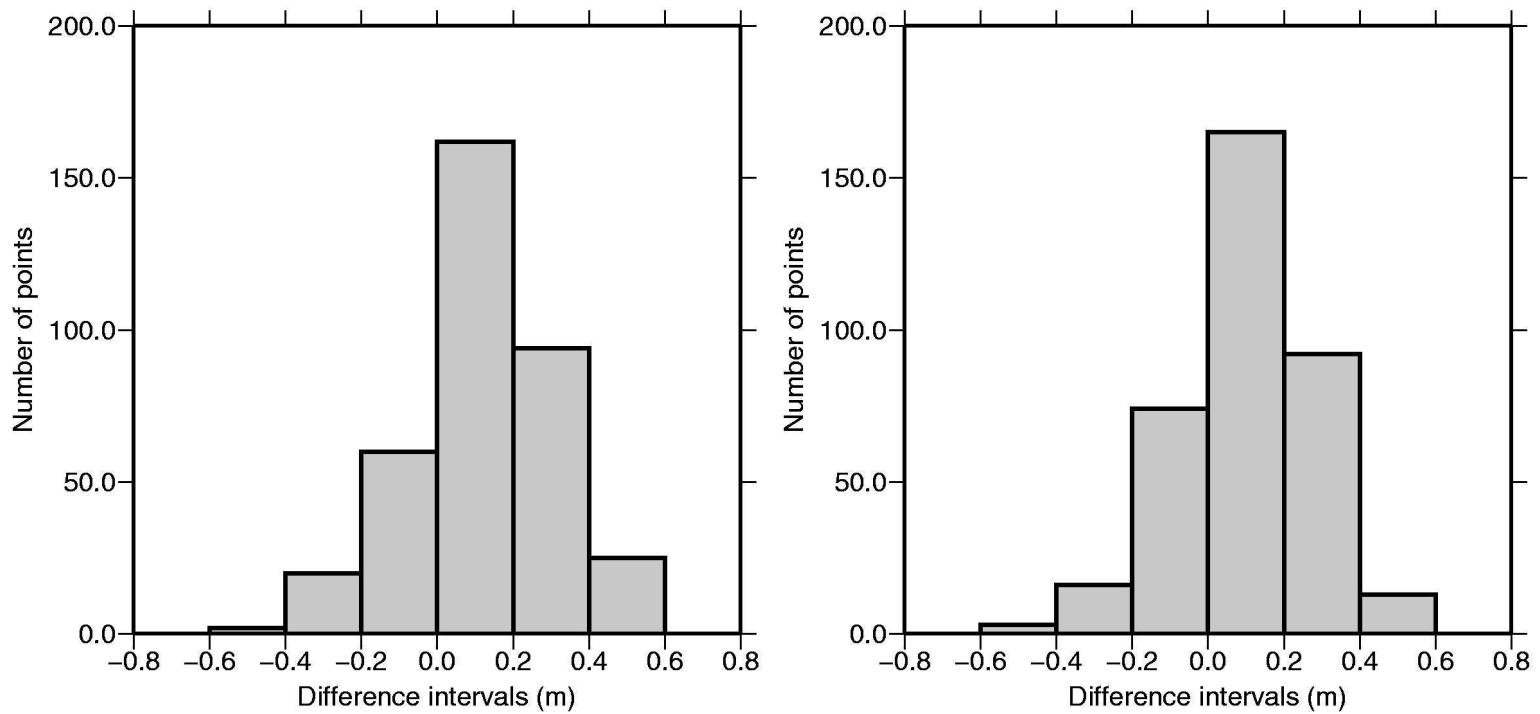

Figure 6.11 - Histogram GPS/leveling and geoid models. 
Table 6.5 - Absolute comparison statistics (units in meters).

\begin{tabular}{lcccc}
\hline & Mean & RMS diff. & Max. & Min. \\
\hline FFT EGM2008 (150) & 0.12 & 0.22 & 0.51 & -0.43 \\
FFT EGM2008 (360) & 0.13 & 0.23 & 0.58 & -0.41 \\
LSC EGM2008 (150) & 0.16 & 0.23 & 0.65 & -0.36 \\
LSC EGM2008 (360) & 0.16 & 0.25 & 0.72 & -0.47 \\
FFT DIR_R3 (150) & 0.11 & 0.21 & 0.49 & -0.44 \\
LSC DIR_R3 (150) & 0.09 & 0.20 & 0.56 & -0.50 \\
FFT TIM_R3 (150) & 0.11 & 0.22 & 0.51 & -0.43 \\
LSC TIM_R3 (150) & 0.09 & 0.20 & 0.58 & -0.47 \\
FFT GOCO03S (150) & 0.12 & 0.22 & 0.51 & -0.43 \\
LSC GOCO03S (150) & 0.09 & 0.20 & 0.54 & -0.47 \\
FFT EIGEN-6C (150) & 0.11 & 0.22 & 0.51 & -0.45 \\
LSC EIGEN-6C (150) & 0.09 & 0.20 & 0.51 & -0.49 \\
\hline
\end{tabular}

Table 6.5 shows that both geoid models (using FFT and LSC) are consistent with GPS/leveling in relation to RMS difference. The differences vary between 0.20-0.22 $\mathrm{m}$ considering only the models up to degree and order 150 . The geoid models based on GOCE data presented results slightly lesser than EGM2008 in terms of mean and RMS difference. The model computed by Least Squares Collocation presented more compatibility than Fast Fourier Transform when GOCE data were used.

\subsubsection{Absolute comparison in the mountains}

In order to evaluate only GPS/leveling stations in the mountain area, 83 points were selected in this region (Figures 6.12). This evaluation pretends to show how is the behavior of both methodologies in a region with less gravity data than in other parts of the state, since the highest differences, according to Figure 6.10, are in this area. 


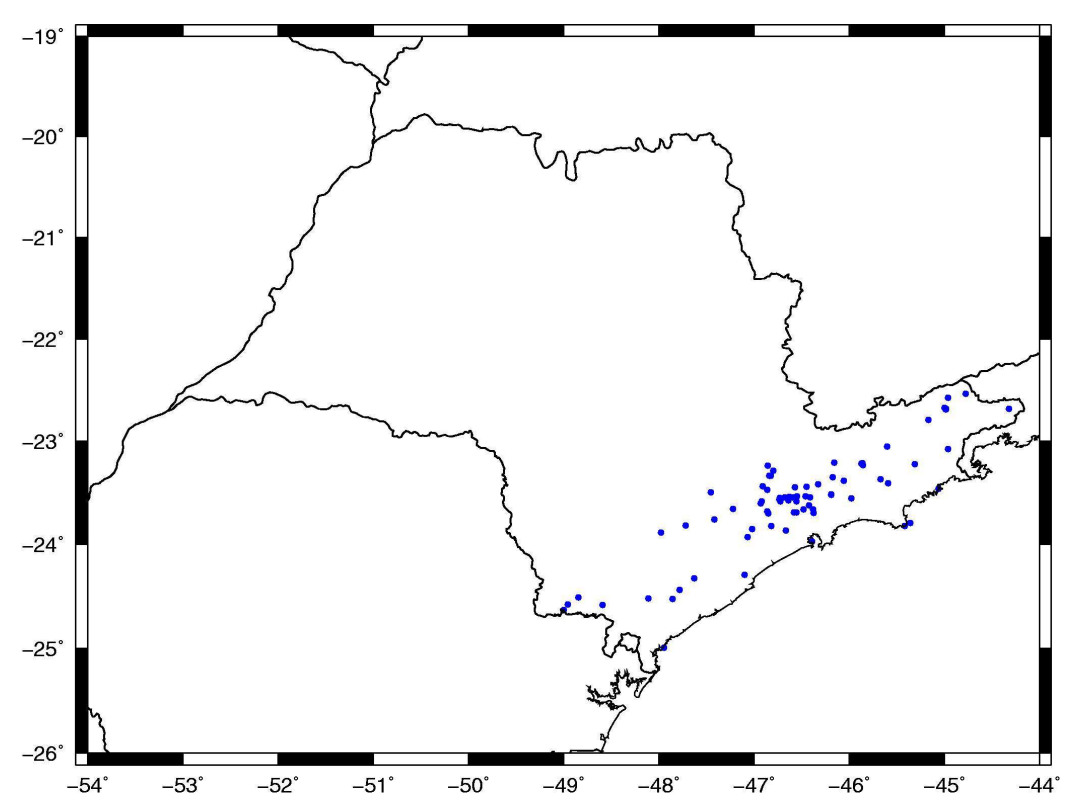

Figure 6.12 - GPS/leveling stations in the mountain area.

Table 6.6 presents the evaluation for the referred area. Considering all models, the results using FFT are more consistent with GPS/leveling than LSC. In terms of RMS difference, the geoid model using EGM2008 presented the highest difference between FFT and LSC $(0.05 \mathrm{~m})$. The obvious conclusion for these differences is that the covariance functions computed did not represent very well this area, since there is a lack of data and the topography is rugged. However, the most important point in this case is that LSC provides the height anomaly $\zeta$ and the GPS/leveling provides the geoid height $N$. Moreover, the higher the altitude, the higher are the differences.

Table 6.6 - Absolute comparison statistics in the mountains (units in meters).

\begin{tabular}{lcccc}
\hline & Mean & RMS diff. & Max. & Min. \\
\hline FFT EGM2008 (150) & 0.17 & 0.25 & 0.49 & -0.43 \\
LSC EGM2008 (150) & 0.25 & 0.30 & 0.59 & -0.33 \\
FFT DIR_R3 (150) & 0.17 & 0.24 & 0.47 & -0.38 \\
LSC DIR_R3 (150) & 0.22 & 0.26 & 0.56 & -0.33 \\
FFT TIM_R3 (150) & 0.18 & 0.25 & 0.50 & -0.40 \\
LSC TIM_R3 (150) & 0.23 & 0.28 & 0.58 & -0.30 \\
FFT GOCO03S (150) & 0.18 & 0.25 & 0.50 & -0.42 \\
LSC GOCO03S (150) & 0.22 & 0.27 & 0.51 & -0.38 \\
FFT EIGEN-6C (150) & 0.18 & 0.25 & 0.50 & -0.42 \\
LSC EIGEN-6C (150) & 0.22 & 0.27 & 0.51 & -0.35 \\
\hline
\end{tabular}




\subsubsection{Relative comparisons}

In relative comparison, pairs of points spaced among $20-50 \mathrm{~km}$ were selected. This range allows evaluating the influence of short wavelength component. A total of 135 pairs were selected. The relative difference was computed applying the following expressions:

$$
\begin{gathered}
N_{G P S 1}-N_{G P S 2}=\Delta N_{G P S} \\
N_{G E O I D 1}-N_{G E O I D 2}=\Delta N_{G E O I D}
\end{gathered}
$$

and is given by:

$$
\text { reldiffe }=\left(\Delta N_{\text {GEOID }}-\Delta N_{G P S}\right) / \text { dist. }
$$

The standard difference value was defined as the mean value resultant of all bases. Figures 6.13 and 6.14 show the pairs of points of the relative comparison in relation to the geoids using FFT and LSC, respectively. In this case, EGM2008 up to degree and order 150 was used as reference field. Table 6.7 presents the relative comparison statistics. 


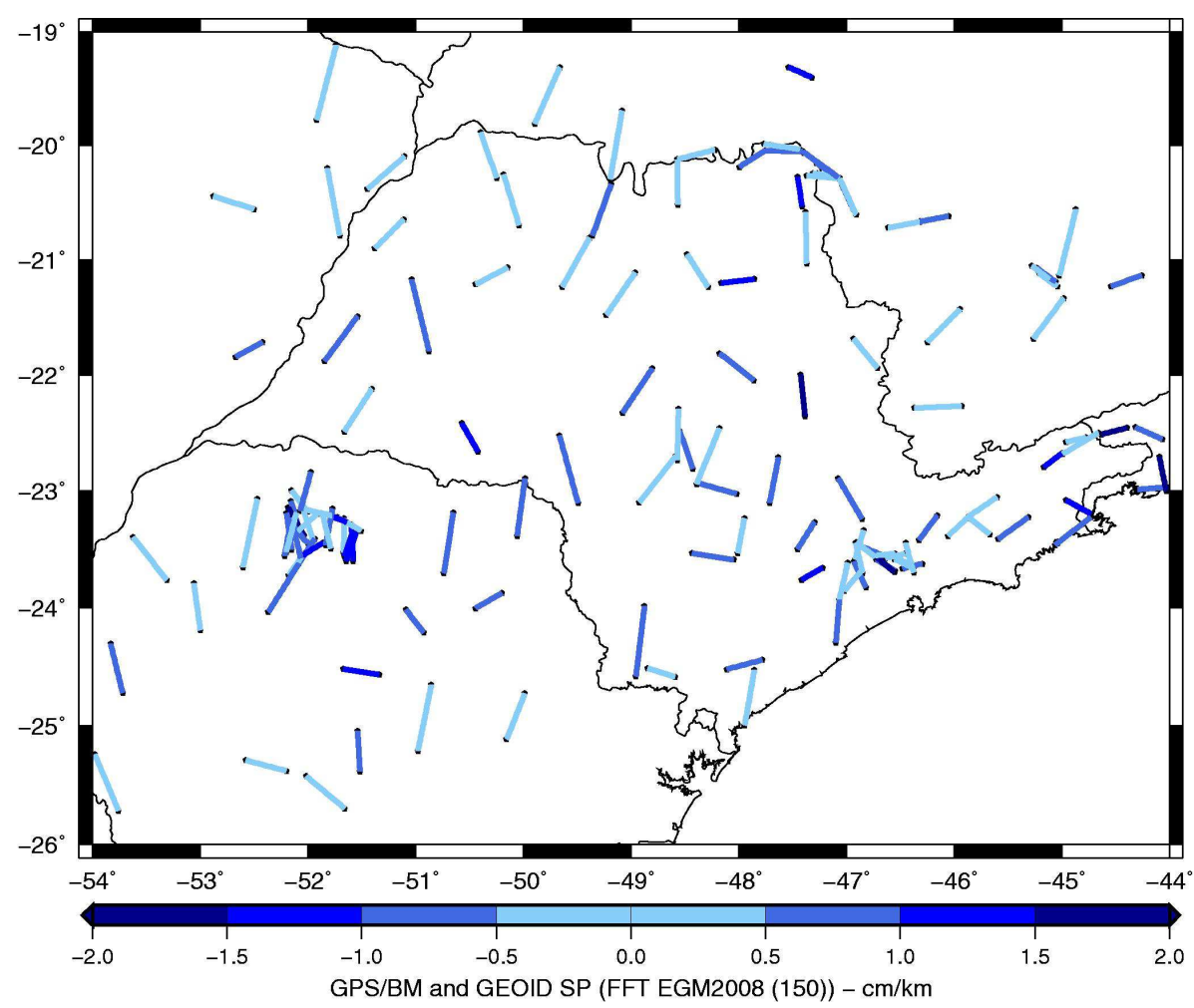

Figure 6.13 - Relative comparison GPS/leveling and GEOIDSP FFT.

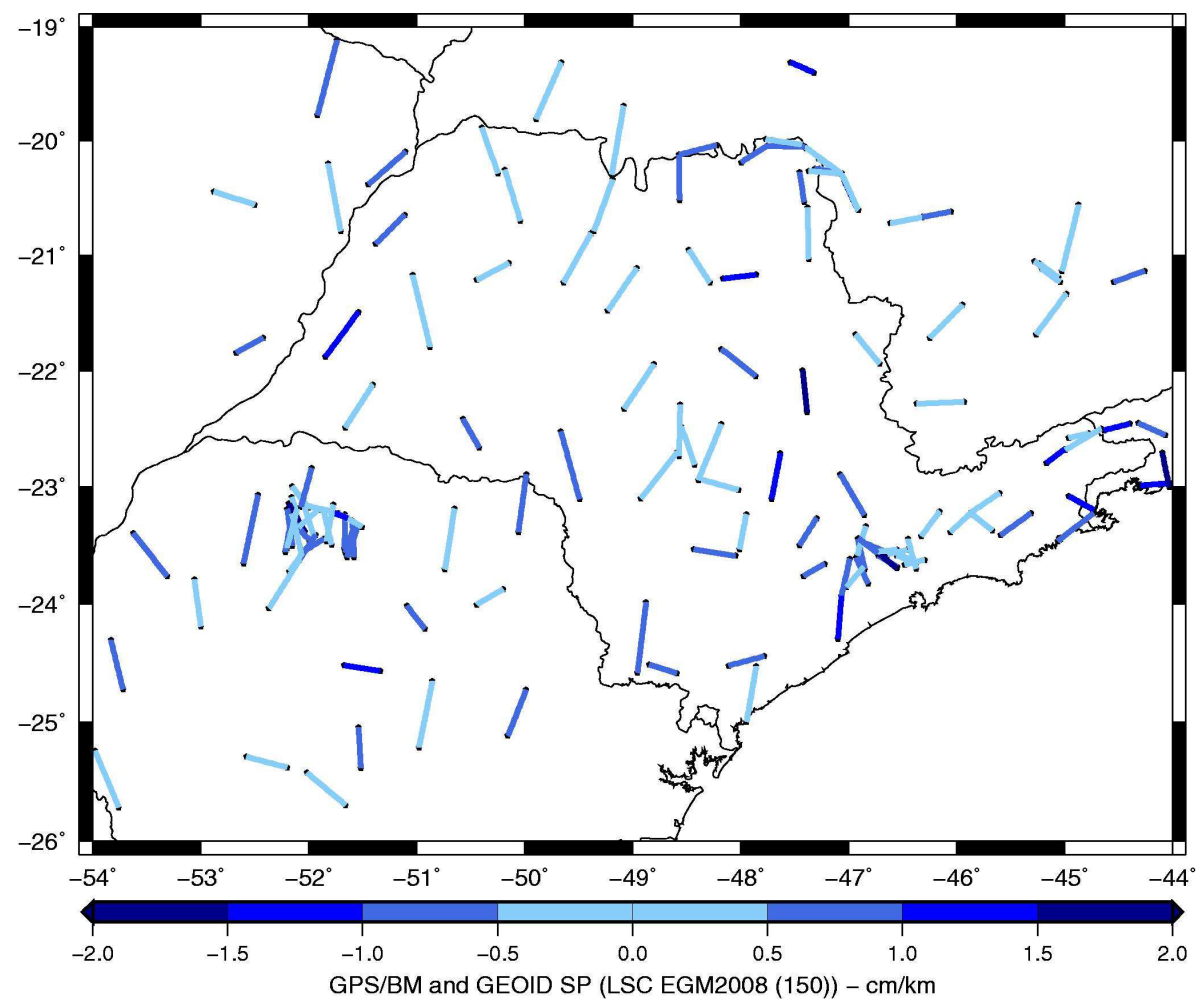

Figure 6.14 - Relative comparison GPS/leveling and GEOIDSP LSC. 
Table 6.7 - Relative comparison statistics.

\begin{tabular}{lcccc}
\hline & $\begin{array}{c}\text { Mean } \\
\text { (cm) }\end{array}$ & $\begin{array}{c}\text { RMS diff. } \\
(\mathbf{c m} / \mathbf{k m})\end{array}$ & $\begin{array}{c}\text { Max. } \\
(\mathbf{c m})\end{array}$ & $\begin{array}{c}\text { Min. } \\
(\mathbf{c m})\end{array}$ \\
\hline FFT EGM2008 (150) & 0.10 & 0.67 & 1.83 & -1.92 \\
FFT EGM2008 (360) & 0.09 & 0.70 & 2.87 & -2.26 \\
LSC EGM2008 (150) & 0.10 & 0.66 & 2.43 & -1.87 \\
LSC EGM2008 (360) & 0.10 & 0.87 & 2.89 & -2.36 \\
FFT DIR_R3 (150) & 0.12 & 0.67 & 2.20 & -1.78 \\
LSC DIR_R3 (150) & 0.09 & 0.66 & 1.98 & -1.54 \\
FFT TIM_R3 (150) & 0.11 & 0.67 & 1.90 & -1.85 \\
LSC TIM_R3 (150) & 0.10 & 0.65 & 2.02 & -1.55 \\
FFT GOCO03S (150) & 0.11 & 0.66 & 2.26 & -1.80 \\
LSC GOCO03S (150) & 0.09 & 0.66 & 1.98 & -1.93 \\
FFT EIGEN-6C (150) & 0.10 & 0.66 & 2.22 & -1.78 \\
LSC EIGEN-6C (150) & 0.10 & 0.66 & 1.94 & -1.80 \\
\hline
\end{tabular}

The statistics in Table 6.7 shows that the comparison for the geoid models up to degree and order 150 are very similar. Regarding the maximum and minimum, FFT models presented higher values than LSC models.

\subsection{GOCE Models Evaluation}

\subsubsection{Comparison in terms of geoid heights}

GOCE based models were compared with GPS/leveling in geoid area in order to evaluate the main models available and to verify the consistency with GPS points. EGM2008 also was employed in the comparisons. The models were computed by the International Centre for Global Earth Model (ICGEM) using the Calculation Service, available at http://icgem.gfz-potsdam.de/ICGEM/ICGEM.html. It has used a regular grid 5' $\times 5$ ', WGS84 as a reference system and the tide free as a tide system. Table 6.8 shows the summary of the spherical harmonic degrees used for each model computation. 
Table 6.8-GGMs degree and order used in the comparisons.

\begin{tabular}{|c|c|c|c|c|}
\hline \multirow{2}{*}{ Models } & \multicolumn{4}{|c|}{$\mathrm{n}$ maximum } \\
\hline & 210 & 224 & 240 & 250 \\
\hline GOCO03S & $\bar{J}$ & & & $\bar{\checkmark}$ \\
\hline DIR_R3 & $\checkmark$ & & $\checkmark$ & \\
\hline TIM_R3 & $\checkmark$ & & & $\checkmark$ \\
\hline EIGEN-6C & $\checkmark$ & & & $\checkmark$ \\
\hline EIGEN-6S & $\checkmark$ & & $\checkmark$ & \\
\hline GOCO02S & $\checkmark$ & & & $\checkmark$ \\
\hline DIR_R2 & $\checkmark$ & & $\checkmark$ & \\
\hline TIM_R2 & $\checkmark$ & & & $\checkmark$ \\
\hline SPW_R2 & $\checkmark$ & & $\checkmark$ & \\
\hline DIR_R1 & $\checkmark$ & & $\checkmark$ & \\
\hline TIM_R1 & $\checkmark$ & $\checkmark$ & & \\
\hline SPW_R1 & $\checkmark$ & & & \\
\hline GOCO01S & $\checkmark$ & $\checkmark$ & & \\
\hline EGM2008 & $\checkmark$ & $\checkmark$ & $\checkmark$ & $\checkmark$ \\
\hline
\end{tabular}

The zero-degree term (in this study it was used $-0.41 \mathrm{~m}$, the same value assumed in the EGM2008) is due to the difference between the geopotential constant $G M$ of the geopotential model and the ellipsoid adopted in all models.

The omission and commission errors in the referred area also were computed for each GOCE-based models. The omission error ensues from the discreteness of, or a lack of resolution in the gravimetric data. This error comprises high-frequency gravity field signals. All gravity field features occurring at scales finer than the GGMs spatial resolution can not be represented by a truncated spherical harmonic series expansion (TORGE, 2001).

Hirt; Featherstone; Marti (2010), describe two ways to model the high-frequency signals not provided by a truncated GGM series expansion, thus reducing the signal omission error. The first methodology is the spectral decomposition approach from regional geoid/quasigeoid modeling via Stokes' or Molodensky's integrals. The second uses residual terrain model (RTM) data for source-modelling of highfrequency gravity field signals. Hirt; Featherstone; Marti (2010) explains that in regions with enough terrestrial gravity data coverage, the first case generally allows more accurate modeling of the gravity field's fine structure than the RTM approach alone. This is because the RTM technique (second option) is usually based on simplifications of the distribution of mass-densities inside the topography. Often, a 
standard rock density is used uniformly for the complete RTM, thus neglecting the impact of any local mass-density variations (HIRT, 2010). In regions with insufficient distribution or scarce availability of gravity data, the local gravimetric refinement of the quasigeoid through first option is of limited use or sometimes even impossible. Particularly in mountainous terrain, second option represents a simple and promising alternative.

Sjöberg (2011) estimated for EGM2008 this error theoretically to $-0.7 \Delta g$ (in millimeter), where $\Delta g$ is the regional mean gravity anomaly in units of mGal. For $\Delta g$ set to 10 and $100 \mathrm{mGal}$ the truncation error becomes -0.007 and $-0.069 \mathrm{~m}$, respectively. The gravity anomaly RMS computed from EGM2008 is $10.32 \mathrm{mGal}$, thus truncation error is $-0.007 \mathrm{~m}$.

When terrestrial data are used in comparison with GGMs, some conditions should be met in their evaluation (HIRT; GRUBER; FEATHERSTONE et al., 2011):

1. It is preferable to use a large amount of terrestrial data, thus making the results in terms of RMS reasonable analysis;

2. It is desirable for the data set to cover a larger area to make the analysis representative, once the spatial resolution of the GOCE-based GGMs is $\sim 100 \mathrm{~km}$;

3. It is beneficial if the comparison data is independent of the data set sourced by the GGMs.

EGM2008 is not completely independent of the terrestrial gravity data because $\sim 80 \%$ was used in the model development. Table 6.9 shows the comparisons results and the estimative of the standard deviation of the omission error $\left(\sigma_{\text {omission }}^{\left(\mathrm{n}_{\max }\right)}\right)$ developed by W.M. Kaula (KAULA, 1966, p. 98). This error is given by:

$$
\sigma_{\text {omission }}^{\left(\mathrm{n}_{\max }\right)}=\sqrt{\sum_{\mathrm{n}=\mathrm{n}_{\max }+1}\left(\sigma_{\mathrm{N}}^{2}\right)} \approx \frac{64}{\mathrm{n}_{\text {max }}}
$$

where $\sigma_{\mathrm{N}}^{2}$ is the degree variance; it is also shown the commission error $\left(\sigma_{\text {commission }}^{\left(\mathrm{n}_{\max }\right)}\right)$ for this region. This last error is independent of the omission error, thus 


$$
\left(\sigma_{\text {commission }}^{\left(\mathrm{n}_{\text {max }}\right)}\right)^{2}=\sigma_{\text {total }}^{2}-\left(\sigma_{\text {cmission }}^{\left(\mathrm{n}_{\text {max }}\right)}\right)^{2}
$$

For a local computation of the geoid height from a particular GGM, the standard deviation of the omission error may be significantly lower or higher because, as previously described, Kaula's rule is a global model for the standard deviation of the omission error. This rule significantly overestimates the power spectral density of the geoid height at the lower frequencies and underestimates at the high frequencies (JEKELI; YANH; KWON, 2009). Thus, it is very difficult to estimate the omission error and the commission error localy. 
Table 6.9 - Statistics of the geoid height (GPS/leveling) minus (GGMs) (units in meters).

\begin{tabular}{lcccccc}
\hline \multicolumn{1}{c}{ GGMs (n=m) } & Mean & $\begin{array}{c}\text { RMS } \\
\text { diff. }\end{array}$ & Max. & Min. & $\sigma_{\text {omission }}^{\left(\mathrm{n}_{\max }\right.}$ & $\sigma_{\text {commision }}^{\left(\mathrm{n}_{\max }\right)}$ \\
\hline GOCO03S (210) & 0.23 & 0.40 & 1.09 & -0.64 & 0.30 & 0.13 \\
DIR_R3 (210) & 0.16 & 0.37 & 1.01 & -0.77 & 0.30 & 0.16 \\
TIM_R3 (210) & 0.18 & 0.38 & 1.01 & -0.73 & 0.30 & 0.16 \\
EIGEN-6C (210) & 0.19 & 0.40 & 1.00 & -0.74 & 0.30 & 0.20 \\
EIGEN-6S (210) & 0.17 & 0.39 & 1.03 & -0.75 & 0.30 & 0.18 \\
GOCO02S (210) & 0.17 & 0.38 & 0.99 & -0.78 & 0.30 & 0.16 \\
DIR_R2 (210) & 0.17 & 0.39 & 1.07 & -0.73 & 0.30 & 0.20 \\
TIM_R2 (210) & 0.17 & 0.34 & 0.99 & -0.78 & 0.30 & 0.23 \\
SPW_R2 (210) & 0.14 & 0.39 & 1.02 & -0.74 & 0.30 & 0.22 \\
DIR_R1 (210) & 0.18 & 0.40 & 1.06 & -0.69 & 0.30 & 0.18 \\
TIM_R1 (210) & 0.13 & 0.37 & 1.00 & -0.81 & 0.30 & 0.18 \\
SPW_R1 (210) & 0.15 & 0.42 & 1.31 & -0.81 & 0.30 & 0.25 \\
GOCO01S (210) & 0.15 & 0.38 & 1.01 & -0.74 & 0.30 & 0.18 \\
EGM2008 (210) & 0.18 & 0.40 & 1.05 & -0.79 & 0.30 & 0.20 \\
TIM_R1 (224) & 0.10 & 0.36 & 0.97 & -0.75 & 0.29 & 0.19 \\
GOCO01S (224) & 0.12 & 0.37 & 0.98 & -0.69 & 0.29 & 0.19 \\
EGM2008 (224) & 0.13 & 0.35 & 1.05 & -0.85 & 0.29 & 0.13 \\
DIR_R3 (240) & 0.11 & 0.33 & 0.97 & -0.83 & 0.27 & 0.15 \\
EIGEN-6S (240) & 0.12 & 0.38 & 1.09 & -0.80 & 0.27 & 0.24 \\
DIR_R2 (240) & 0.18 & 0.39 & 1.06 & -0.82 & 0.27 & 0.22 \\
SPW_R2 (240) & 0.13 & 0.38 & 1.01 & -0.75 & 0.27 & 0.24 \\
DIR_R1 (240) & 0.07 & 0.31 & 0.83 & -0.64 & 0.27 & 0.13 \\
EGM2008 (240) & 0.08 & 0.31 & 0.93 & -0.62 & 0.27 & 0.13 \\
GOCO03S (250) & 0.17 & 0.34 & 1.00 & -0.67 & 0.26 & 0.13 \\
TIM_R3 (250) & 0.11 & 0.32 & 1.01 & -0.74 & 0.26 & 0.15 \\
EIGEN-6C (250) & 0.08 & 0.30 & 0.87 & -0.64 & 0.26 & 0.13 \\
GOCO02S (250) & 0.12 & 0.34 & 0.96 & -0.76 & 0.26 & 0.19 \\
TIM_R2 (250) & 0.12 & 0.35 & 1.01 & -0.77 & 0.26 & 0.19 \\
EGM2008 (250) & 0.08 & 0.30 & 0.92 & -0.59 & 0.26 & 0.13 \\
\hline
\end{tabular}


Looking at the statistics in Table 6.9, one can see that release 3 from the model based on the direct approach (DIR) and the release 1 from TIM approach presented the smallest RMS $(0.37 \mathrm{~m})$ concerning only the degree and order 210 . For spherical harmonic degrees 224 and 240, EGM2008 presented the smallest RMS $(0.35 \mathrm{~m}$ and $0.31 \mathrm{~m}$ ), respectively, while for $\mathrm{n}=\mathrm{m}=250$ this model and EIGEN-6C have the same results $(0.30 \mathrm{~m})$. DIR model, release 3 , reduced the RMS errors in $0.02 \mathrm{~m}(5.40 \%$ reduction) in comparison with release 1 and $0.03 \mathrm{~m}$ (8.11\% reduction) with release 2 , for $n=m=210$. With respect to $n=m=240$, releases 1 and 3 present slightly close results. Concerning TIM model, the release 2 presented the smaller result in terms of RMS difference $(0.34 \mathrm{~m})$ for $n=m=210$. Regarding SPW model, release 1 presented the less consistent result $(0.42 \mathrm{~m})$ for $\mathrm{n}=\mathrm{m}=210$ and the difference in relation to the second release is $0.03 \mathrm{~m}$.

Figure 6.15 shows the results in a graphical form. The solid black line (EGM2008 results) is represented in all plots to serve as a reference. For degree and order 210 , EGM2008 presented less consistent results than the other models (except DIR_R2 and SPW_R1). This suggests that GOCE-based models present a better performance for the spectral band of spherical harmonics up to degree 210 in comparison to EGM2008. For $n=m=250$, EGM2008 and EIGEN-6C provided the smallest results $(0.31 \mathrm{~m})$. 

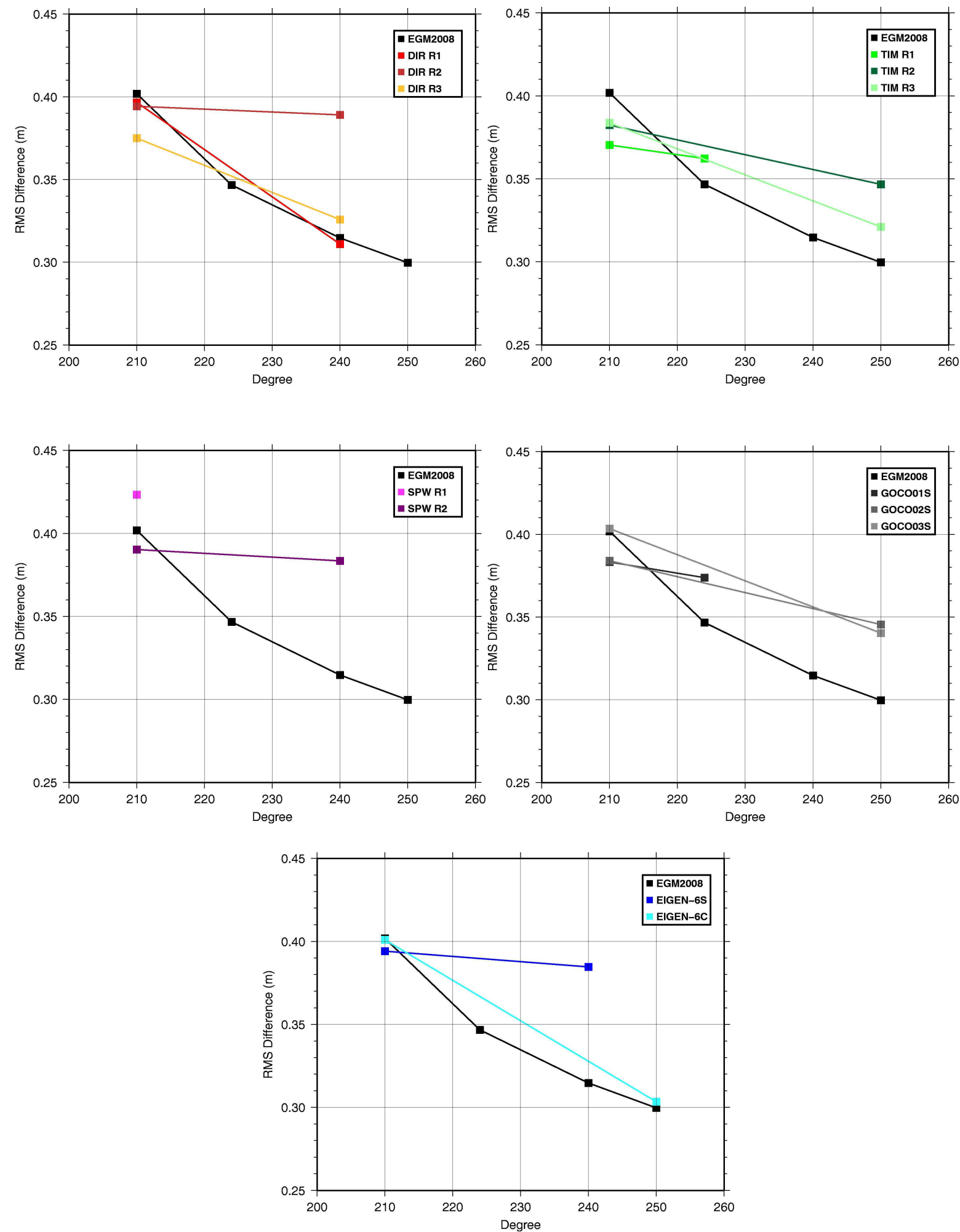

Figure 6.15 - RMS of the geoid height (GPS/leveling) minus (GGMs). 


\subsubsection{Comparison in terms of gravity disturbances}

In this second assessment, the gravity disturbance from terrestrial gravity data was compared with the GOCE-based models gravity disturbance. The 'observed' gravity disturbances have been computed by subtracting the normal gravity and the observed gravity.

This topic shows two comparisons. In the first one it was added the RTC to GGMs gravity disturbances. This correction for each station was computed using TC program (FORSBERG, 1984) and the digital terrain model SAM3s_V2 (§ 5.3). The RMS, related to RTC, is reported in Table 6.10 , and Table 6.11 shows the results without the RTC for degree and order up to 210 . The omission error comprises highfrequency gravity field signals that cannot be represented by a truncated spherical harmonic series expansion of the GGMs (TORGE, 2001). The RTC estimate these signals, so omission errors are modelled. The use of RTC omission error corrections are comparable to the RTM omission error corrections and it should be stated which data set was used as long-wavelength reference in the RTC computations. 
Table 6.10 - Statistics of gravity disturbance differences (observed minus models) with RTC (units in $\mathrm{mGal})$.

\begin{tabular}{|c|c|c|c|c|}
\hline GGMs $(n=m)$ & Mean & $\begin{array}{l}\text { RMS } \\
\text { diff. }\end{array}$ & Max. & Min. \\
\hline GOCO03S (210) & 0.75 & 10.42 & 116.41 & -142.74 \\
\hline DIR_R3 (210) & 1.01 & 10.52 & 116.02 & -143.15 \\
\hline TIM_R3 (210) & 0.72 & 10.47 & 117.28 & -142.78 \\
\hline EIGEN-6C (210) & 0.42 & 10.60 & 116.82 & -140.94 \\
\hline EIGEN-6S (210) & 0.92 & 10.63 & 115.09 & -140.31 \\
\hline GOCO02S (210) & 0.84 & 10.34 & 116.47 & -137.51 \\
\hline DIR_R2 (210) & 0.82 & 10.65 & 115.71 & -141.71 \\
\hline TIM_R2 (210) & 0.79 & 10.37 & 116.78 & -136.89 \\
\hline SPW_R2 (210) & 0.69 & 10.73 & 112.44 & -138.57 \\
\hline DIR_R1 (210) & 0.51 & 10.66 & 118.54 & -144.77 \\
\hline TIM_R1 (210) & 1.36 & 11.06 & 116.42 & -142.46 \\
\hline SPW_R1 (210) & -0.98 & 11.75 & 113.66 & -141.20 \\
\hline GOCO01S (210) & 1.37 & 11.03 & 115.78 & -143.48 \\
\hline EGM2008 (210) & 0.35 & 10.66 & 117.49 & -138.84 \\
\hline TIM_R1 (224) & 1.05 & 26.56 & 135.63 & -126.94 \\
\hline GOCO01S (224) & 1.47 & 11.22 & 113.97 & -144.05 \\
\hline EGM2008 (224) & 0.77 & 10.12 & 113.74 & -137.49 \\
\hline DIR_R3 (240) & 1.17 & 10.31 & 111.40 & -147.09 \\
\hline EIGEN-6S (240) & 1.32 & 11.23 & 110.58 & -144.31 \\
\hline DIR_R2 (240) & 1.22 & 11.19 & 110.44 & -145.76 \\
\hline SPW_R2 (240) & 0.80 & 10.74 & 111.37 & -139.98 \\
\hline DIR_R1 (240) & -0.69 & 10.21 & 116.89 & -143.68 \\
\hline EGM2008 (240) & 0.43 & 10.16 & 114.71 & -136.53 \\
\hline GOCO03S (250) & 1.12 & 10.13 & 110.72 & -145.93 \\
\hline TIM_R3 (250) & 1.09 & 10.28 & 111.50 & -146.37 \\
\hline EIGEN-6C (250) & 0.72 & 9.69 & 116.34 & -139.74 \\
\hline GOCO02S (250) & 1.14 & 10.29 & 111.75 & -138.77 \\
\hline TIM_R2 (250) & 1.10 & 10.38 & 111.56 & -138.32 \\
\hline EGM2008 (250) & 0.62 & 9.72 & 115.66 & -136.95 \\
\hline
\end{tabular}


Table 6.11 - Statistics of gravity disturbance differences (observed minus models) without RTC (units in $\mathrm{mGal})$.

\begin{tabular}{lcccc}
\hline \multicolumn{1}{c}{ GGMs (n=m) } & Mean & $\begin{array}{c}\text { RMS } \\
\text { diff. }\end{array}$ & Max. & Min. \\
\hline GOCO03S (210) & 0.33 & 16.46 & 121.29 & -119.43 \\
DIR_R3 (210) & 0.59 & 16.57 & 120.90 & -119.84 \\
TIM_R3 (210) & 0.30 & 16.47 & 122.16 & -119.47 \\
EIGEN-6C (210) & 0.00 & 16.48 & 121.70 & -117.63 \\
EIGEN-6S (210) & 0.50 & 16.61 & 119.97 & -117.00 \\
GOCO02S (210) & 0.42 & 16.43 & 121.35 & -114.20 \\
DIR_R2 (210) & 0.40 & 16.56 & 120.59 & -118.40 \\
TIM_R2 (210) & 0.37 & 16.43 & 121.66 & -113.58 \\
SPW_R2 (210) & 0.27 & 16.72 & 117.32 & -115.26 \\
DIR_R1 (210) & 0.09 & 16.58 & 123.42 & -121.46 \\
TIM_R1 (210) & 0.93 & 17.06 & 121.30 & -119.15 \\
SPW_R1 (210) & 0.55 & 17.50 & 118.54 & -117.89 \\
GOCO01S (210) & 0.42 & 16.43 & 121.35 & -114.20 \\
EGM2008 (210) & -0.08 & 16.47 & 122.37 & -115.53 \\
\hline
\end{tabular}

In general, the results in terms of RMS, (Table 6.10) are very close, except the model TIM_R1 (26.56 mGal). GOCE approaches (DIR, TIM and SPW) present slight decrease in terms of RMS considering $n=m=210$, but the model TIM_R3 presented an increase when compared to release 2. The results obtained for DIR and TIM, release 3 are smaller than EGM2008. Furthermore, GOCO02S and GOCO03S also presented values lower than EGM2008. The results of the latest releases improved a little bit compared to EGM2008.

Comparing Tables 6.10 and 6.11, the use of RTC reduced the RMS errors in $\sim 6.00$ mGal ( 40\%). The significant improvement occurred close to the coast line (in a mountain region). Some comparisons involving EGM2008 were carried out by Hirt; Featherstone and Marti (2010) and Hirt, Gruber and Featherstone (2011), and demonstrated the improvement when applying RTC. Figure 6.16 depicts the differences, for five models, up to degree and order 210 . On the left, the results without the RTC and, on the right, applying the correction. 

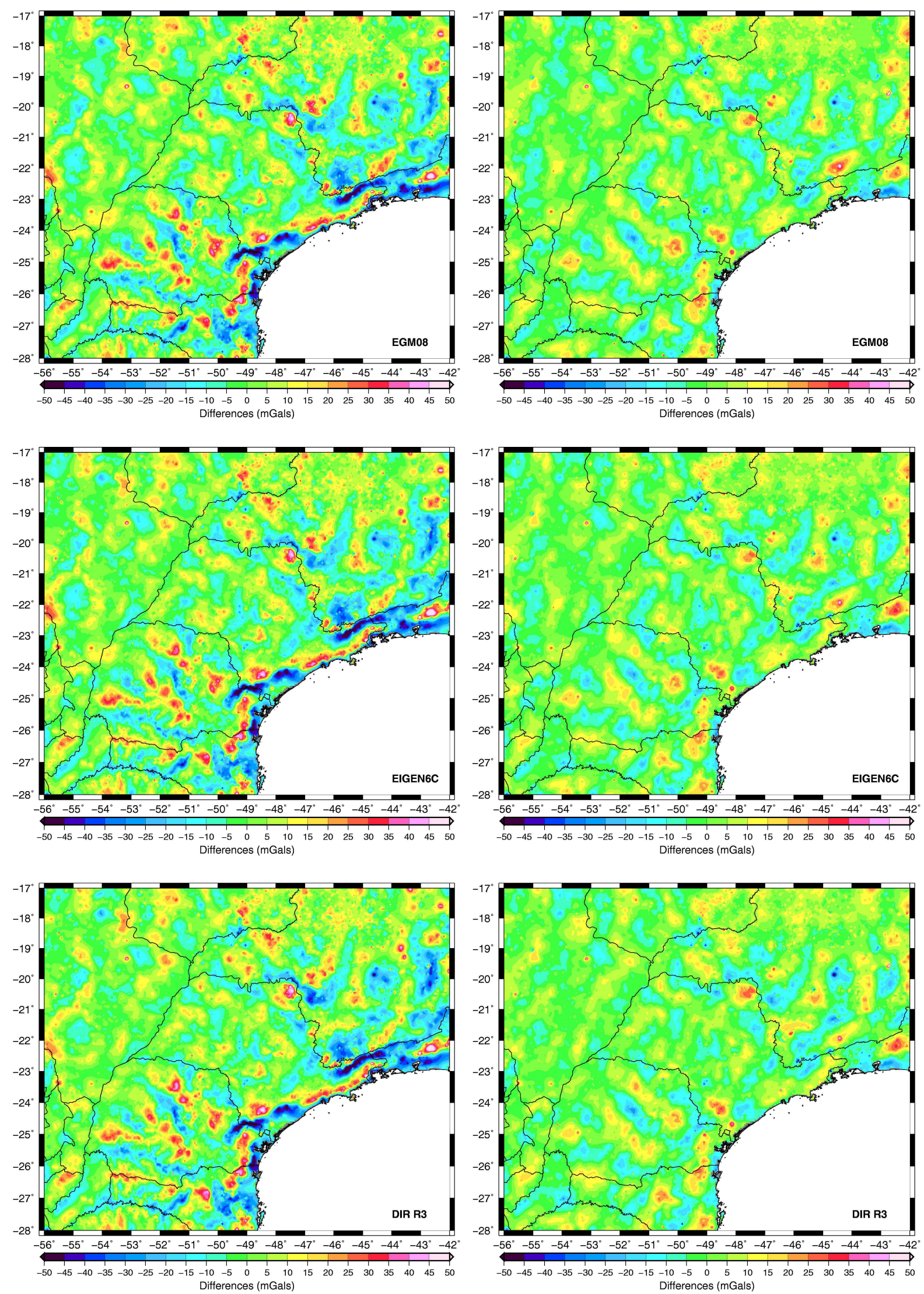

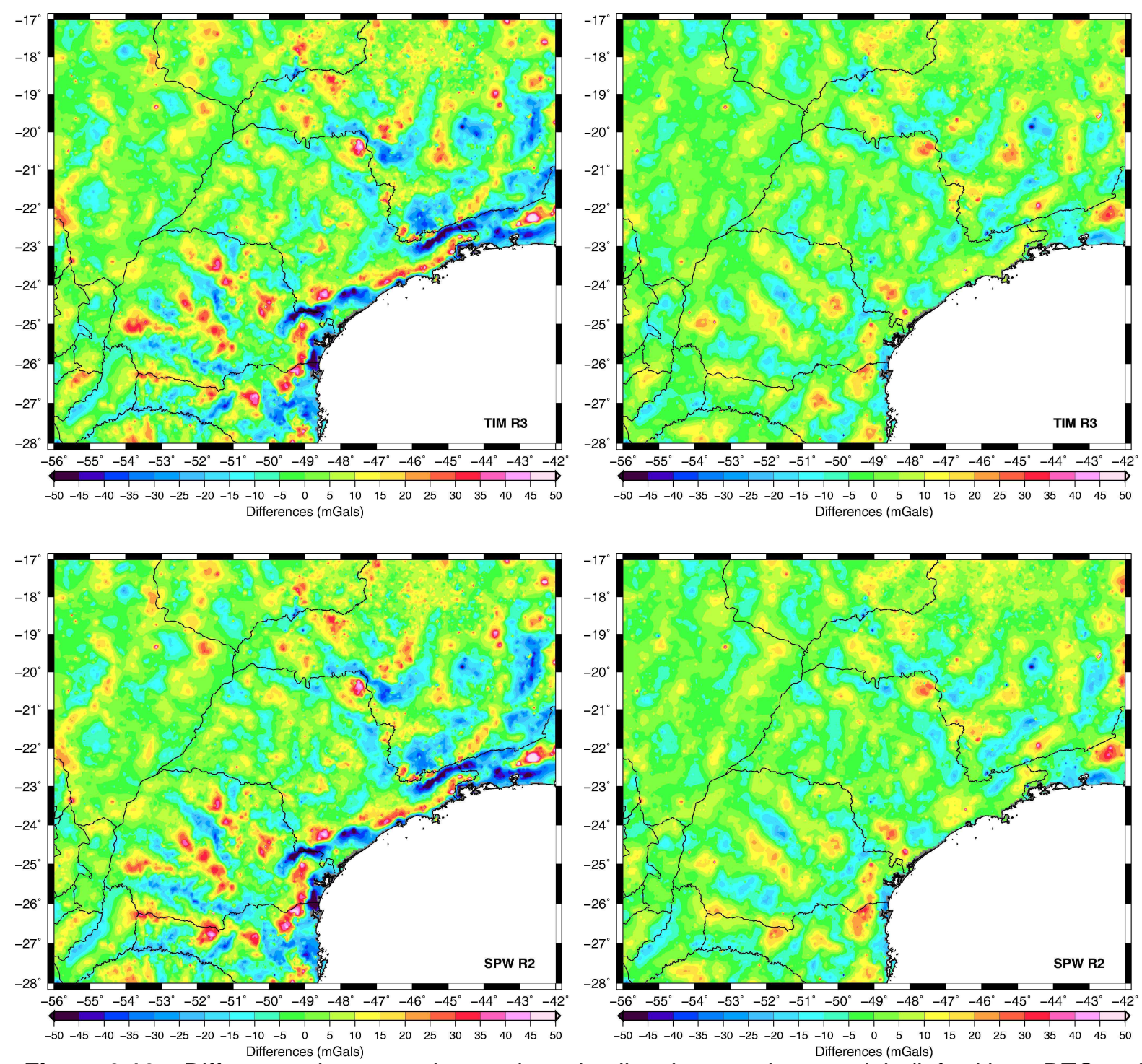

Figure 6.16 - Differences between observed gravity disturbance minus models (left without RTC and right with RTC). 


\section{CONCLUSIONS AND RECOMMENDATIONS}

The geoid model in the State of São Paulo was computed using two different methodologies (Stokes' integral applying Fast Fourier Transform and Least Squares Collocation). The computation was performed using EGM2008 (degree and order 150 and 360), GOCE-based models (DIR_R3 and TIM_R3), GOCO03S and EIGEN6C (degree and order 150) as the reference field for long wavelength component. Three comparisons were carried out to verify the quality and consistency of the models. In the first evaluation, the geoid height residual computed by FFT and LSC was compared for the same degree and order. Regarding all geoid models, for $\mathrm{n}=\mathrm{m}=150,65-70 \%$ of the area has differences between $0.00 \mathrm{~m}$ and $\pm 0.10 \mathrm{~m}, 30$ $35 \%$ between $\pm 0.10 \mathrm{~m}$ and $\pm 0.20 \mathrm{~m}$ and $0.40-1.00 \%$ larger than $\pm 0.20 \mathrm{~m}$. In the comparison using $\mathrm{n}=\mathrm{m}=360$ the statistics are: $91.10 \%$ of the area has difference between $0.00 \mathrm{~m}$ and $\pm 0.10 \mathrm{~m}, 7.90 \%$ between $\pm 0.10 \mathrm{~m}$ and $\pm 0.20 \mathrm{~m}$ and $1.00 \%$ larger than $\pm 0.20 \mathrm{~m}$. In this case, differences larger than $0.20 \mathrm{~m}$ were found in the places that there are some gaps in terms of gravity data. It can be concluded that in the most part of the State of São Paulo both methodologies are consistent in the order of $0.10 \mathrm{~m}$.

In the second comparison the geoid models were verified by comparing GPS/leveling points in the absolute way. This is a very powerful tool to analyze the consistency of each other. It was used 363 points distributed all over the area. The differences in terms of root mean square are between $0.20 \mathrm{~m}$ and $0.23 \mathrm{~m}$ for models up to 150 . The geoid models based on GOCE data presented better consistency with GPS/leveling points than EGM2008. Furthermore, LSC models fitted better than FFT for the GOCE models. However, in the absolute comparison in the mountains, FFT is more consistent than LSC. In this case, the RMS differences for the geoid models using FFT are between $0.24 \mathrm{~m}$ and $0.25 \mathrm{~m}$, while the LSC models are between 0.26 $\mathrm{m}$ and $0.30 \mathrm{~m}$. The reason for LSC be less compatible than FFT can be explained by the lack of data and the topography that affected the LSC computation, since the gravity anomalies are highly correlated with the topography and can influence the modelling of covariance functions. Again, the models based on GOCE data presented similar results in relation to EGM2008 in the mountain area. In the third evaluation, relative comparisons showed that FFT and LSC $(n=m=150)$ presented 
very close results. In terms of RMS, the differences are between 0.65 and 0.67 $\mathrm{cm} / \mathrm{km}$.

The aim of the item 6.4 was to evaluate and to analyze GOCE-based models from terrestrial data. As a first evaluation, the geoid height from GPS/levelling and from GOCE-based models were used. This is a powerful evaluation technique to check GGMs performance. The results showed a gain in accuracy of the GOCE-based models with respect to EGM2008 (spherical harmonic degree 210). In the GPS/leveling comparisons, for degree and order 210, GOCE models improved over EGM2008. Out of 13 models evaluated, 12 of them presented findings smaller or equal than EGM2008 in terms of RMS. Comparisons at higher degrees (e.g. degree and order 240) include the effect of gravity attenuation in GOCE models and the use of terrestrial or predicted gravity in EGM2008.

TIM_R2 model provided the best consistency related to GPS/leveling $(0.34 \mathrm{~m}$ for degree and order 210). The release 3 solution DIR_R3 (degree and order 210) improved significantly against those of releases 1 and 2. Similarly, release 2 of the SPW solution presented better results than release 1. For degree and order 250, EGM2008 and EIGEN-6C presented the smallest value $(0.29 \mathrm{~m})$. It is worth mentioning that the models loose accuracy for resolutions above degree and order 210. This suggests that GOCE-based models do not present the same performance for the spectral band of spherical harmonics above degree 210. This agrees with demonstrations of similar results in other regions (Gruber et al., 2011).

In the second evaluation, gravity disturbances derived from terrestrial gravity data and from GOCE-based models were compared. The results indicate slight decrease in the latest releases DIR_R3 (10.52 mGal), TIM_R3 (10.47 mGal) and GOCO02S (10.34 mGal) in comparison with EGM2008 (10.66 mGal) for degree and order 210. Also, the RTC reflected its contribution to reduce the RMS, especially in the mountain area, where the highest discrepancies were found. Considering only this area, the mean improvement, considering all models, was about $45 \%$. The models DIR and TIM, release 3, presented a similar performance in both comparisons. While DIR_R3 fitted slightly better with GPS/leveling $(0.39 \mathrm{~m}$ against $0.40 \mathrm{~m})$, TIM_R3 performed somewhat better than DIR_R3 in terms of terrestrial gravity data (10.47 mGal against $10.52 \mathrm{mGal}$ ). 
In summary, the comparisons involving GOCE-based models presented significant findings. The models DIR_R3 and TIM_R3 showed excellent results in all comparisons. It can be assumed that GOCE mission moves to achieve the goals. Deeper investigations are expected to be made in Brazil and also in South America to show the contribution of GOCE mission.

It is worth mentioning that a study in terms of tide system should be carried out, since the spirit leveling network is referred to the mean tide and the geopotencial models are tide free. Also, it is important to cover the lacks of gravity data in the State of São Paulo and surrounding area. In this way, the geoid model would be improved. The geoid model computation in an area with poor gravity data distribution could be an opportunity to verify the LSC methodology and also the covariance functions. LSC could be tested in the Amazon region. In most part of the forest there is no gravity data, however, close to the rivers there are a substantial quantity of data. Furthermore, this region is quite flat, which could be a positive indication in determining the covariance functions. The use of GPS and gravity data in the LSC determination could be undertaken to verify how GPS can contributes in the geoid model computation. 


\section{REFERENCES}

ALMEIDA FILHO, F.G.V. Variação temporal do campo gravitacional detectado pelo satélite GRACE: aplicação na bacia Amazônica. 2009. 146p. PhD Thesis Escola Politécnica, Universidade de São Paulo, São Paulo, 2009.

ANDERSEN, O.B. The DTU10 Gravity field and Mean sea surface. Second international symposium of the gravity field of the Earth (IGFS2), Fairbanks, Alaska. 2010.

ARABELOS, D.; TSCHERNING C.C. A comparison of recent Earth gravitational models with emphasis on their contribution in refining the gravity and geoid at continental or regional scale. Journal of Geodesy, v.84, p. 643-660. Doi: 10.1007/s00190-010-0397-z, 2010.

AUBIN, J.P. Applied Functional Analysis. New York: Wiley 423p, 1979.

BAKER, E.M. A finite element model of the Earth's anomalous gravitational potential, OSU Rep 391, Dept Geod Sci Surv, Ohio State University, Columbus, 1988.

BIANCALE, R. 1ère Ecole d'Eté du GRGS. Forcalquier: France, 2002. (Notes of Summer Course, CNES/GRGS).

BLITZKOW, D. O problema do valor de contorno da geodésia: resultados práticos para a América do Sul. 1996. 81p. Tese (Livre Docência) - Escola Politécnica, Universidade de São Paulo, São Paulo, 1996.

BLITZKOW, D. CINTRA, J. P, FORTES L.P.S. A contribution to the geoid determination. In: Recent Geodetic and Gravimetric research in Latin America. Edited by W. Torge. Springer-Verlag. Berlin, 1991.

BLITZKOW, D.; et al. An attempt for an Amazon geoid model using Helmert gravity anomaly. In: Michael G. Sideris. (Org.). Observing our Changing Earth. 1 ed. : Springer-Verlag, 2008, v. 133, p. 187-194, 2008.

BLITZKOW, D.; et al. A new version of the geoid model for South America. Second International Symposium of the International Gravity Field Service - IGFS2 20 22 September 2010 Fairbanks, Alaska, 2010.

BOTTONI, G.P.; BARZAGHI, R. Fast collocation. Bulletin Géodésique. v. 67, p. 119-126, 1993.

BRACEWELL, R. The Fourier transform and its applications. $3^{\text {rd }}$ edition, McGraw Hill, New York, 1986. 
BRUINSMA, S.L.; et al. GOCE gravity field recovery by means of the direct numerical method, presented at the ESA Living Planet Symposium, 27th June - 2nd July, Bergen, Norway, 2010, See also: earth.esa.int/GOCE.

DRINKWATER, M.R.; et al. The GOCE gravity mission: ESA's first core earth explorer, Proceedings... of 3rd International GOCE User Workshop, 6-8 November, 2006, Frascati, Italy, ESA SP-627, ISBN 92-9092-938-3, p.1-8, 2007.

DURBIN, J. The fitting of the time series models. Revue de L'institut International de Statistique, v. 28, n. 3, p. 233-244, 1960.

ELLMANN, A. SHGEO software packages-An UNB Application to StokesHelmert Approach for Precise Geoid Computation, reference manual I , 36 p. 2005a.

http://gge.unb.ca/Research/GRL/GeodesyGroup/SHGeo_package/Manual/SHGeo_ manual_I_v2.pdf. Access in 2012.

ELLMANN A. SHGEO software packages-An UNB Application to Stokes-Helmert Approach for Precise Geoid Computation, reference manual II, 43 p. 2005b Available in: http://gge.unb.ca/Research/GRL/GeodesyGroup/SHGeo_package. Access in 2012.

ELLMANN, A.; VANÍČEK, P. UNB applications of Stokes-Helmert's approach to geoid computation. Journal of Geodynamics, v. 43, p. 200-213, 2007.

ESA - ESA's gravity mission - GOCE. BR-2009. Revised June 2006. ESA Publications Division. ESTEC, Noordwijk, The Netherlands, 2006.

EWING, M. Marine Gravimetric Methods and Surveys. Proceedings of the American Philosophical Society, v. 79, n. 1, 1938.

FARR, T.G.; et al. The Shuttle Radar Topography Mission, Reviews of Geophysics, v. 45,2007 , DOI:10.1029/2005RG000183.

FEATHERSTONE, W.E. Software for computing five existing types of deterministically modified integration kernel for gravimetric geoid determination. Computer \& Geosciences, v. 29, p. 183-193, 2003. Software available at: http://www.iamg.org/CGEditor/index.html

FEATHERSTONE, W.E.; EVANS, J.; OLLIVER, J. A Meissl-modified Vanícek and Kleusberg kernel to reduce the truncation error in gravimetric geoid computations. Journal of Geodesy, v.72, p. 154-160, 1998.

FEATHERSTONE, W.E.; et al. The AUSGeoid98 geoid model of Australia: data treatment, computations and comparisons with GPS-levelling data. Journal of Geodesy, v. 75, p. 313-330, 2001.

FEATHERSTONE, W.E.; SIDERIS, M.G. Modified kernels in spectral geoid determination: first results from Western Australia. In: R. Forsberg and M. Feissl and R. Dietrich (Ed.). Geodesy on the move: gravity, geoids, geodynamics, and 
Antarctica, Berlin: Springer-Verlag. International Association of Geodesy Symposia , 119:188-193, 1998.

FEHRINGER, M.; et al. GOCE and its gravity measurements systems, ESA Bulletin 133, p. 14-23, February, 2008.

FLOBERGHAGEN, R.; et al. GOCE's measurements of the gravity field and beyond and its gravity measurements systems, ESA Bulletin 133, 24-31, February, 2008.

FORSBERG, R. A study of terrain reductions, density anomalies and geophysical inversion methods in gravity field modelling. Report 355, Department of Geodetic Science and Surveying, Ohio State University, Columbus, 1984.

FORSBERG. R.; TSCHERNING C.C. An overview manual for the GRAVSOFT geodetic gravity field modelling programs. $2^{\text {nd }}$ edition, Denmark, 2008.

FÖRSTE C.; et al. A mean global gravity field model from the combination of satellite mission and altimetry/gravimetry surface data - EIGEN-GL04C, Geophysical Research Abstracts, v. 8, 2006.

FÖRSTE C.; et al. EIGEN-6 - A new combined global gravity field model including GOCE data from the collaboration of GFZ-Potsdam and GRGS-Toulouse, Geophysical Research Abstracts, v. 13, EGU General Assembly, 2011.

GOIGINGER $\mathrm{H}$; ; et al. The combined satellite-only global gravity field model GOCO02S, presented at the 2011 General Assembly of the European Geosciences Union, Vienna, Austria, April 4-8, 2011.

GRACE - Gravity recovery and climate experiment: Science and mission. Requirements document, revision A, JPLD-15928, NASA's Earth System Science Pathfinder Program, 1998.

GRUBER T.; et al. Validation of GOCE gravity field models by means orbit residuals and geoid comparisons. Journal of Geodesy, 2011, DOI: 10.1007/s00190-011-04867.

HASTING, D.A.; DUNBAR, P.K. Global land one-kilometer base elevation (GLOBE) Digital Elevation Model Documentation, v 1, National Oceanic and Atmospheric Administration, National Geophysical Data Center, 1999.

HECK, B.; GRÜNINGER, W. Modification of Stokes's integral formula by combining two classical approaches. Poster Presentation in IUGG General Assembly, Vancouver, 1987.

HEISKANEN, W.A.; MORITZ, H. Physical Geodesy, W. H. Freeman, San Francisco, CA, 364 p 1967.

HIRT, C. Prediction of vertical deflections from high-degree spherical harmonic synthesis and residual terrain model data. Journal of Geodesy, v. 84 n. 3, p. 179190, 2010. DOI: 10.1007/s00190-009-0354-x. 
HIRT, C.; FEATHERSTONE, W.E.; MARTI U. Combining EGM2008 and SRTM/DTM2006.0 residual terrain model data to improve quasigeoid computations in mountains area devoid of gravity data. Journal of Geodesy, v. 84, p. 557-567, 2010. DOI: 10.1007/s00190-010-0395-1.

HIRT, C.; GRUBER, T.; FEATHERSTONE, W.E. Evaluation of the first GOCE static gravity field models using terrestrial gravity, vertical deflections and EGM2008 quasigeoid heights. Journal of Geodesy, v. 85 n. 10, p. 723-740, 2011. DOI: 10.1007/s00190-011-0482-y.

HOFMANN-WELLENHOF, B.; MORITZ, H. Physical Geodesy. $2^{\text {nd }}$ Edition, New York: Springer. 403 p, 2006.

HOLMES, S.; PAVLIS, N.K. Earth Gravitational Model 2008 (EGM2008). Available in: http://earthinfo. nga.mil/GandG/wgs84/gravitymod/egm2008/first release.html. Access in: October 2012.

IBGE. Ajustamento simultâneo da rede altimétrico de alta precisão do sistema brasileiro geodésico brasileiro. Relatório, Rio de Janeiro, 62p. Available in: <http://www.ibge.gov.br/home/geociencias/geodesia/altimetrica.shtm> access in October 2011.

JEKELI, C. Potential theory and static gravity field on the Earth In: Schubert G. (Ed.) Geodesy, treatise on geophysics v. 3 p 11-43, 2009.

JEKELI, C.; YANH, H.J.; KWON, J.H. Evaluation of EGM08 - globally and locally in South Korea. Newton's Bulletin, Publication the International Association of Geodesy and International Gravity Field Service. v. 4, p. 38-49, 2009. ISSN $1810-$ 8555.

KAULA, W.M. Theory of Satellite Geodesy. Blaisdell Publ. Co., Waltham, Mass, 1966.

LEMOINE, F.G.; et al. New high-resolution model developed for Earth' gravitational field, EOS, Transactions AGU, 79, 9, March 3, v. 113, p. 117-118. 1998a.

LEMOINE, F.G.; et al. The development of the joint NASA GSFC and the National Imagery and Mapping Agency (NIMA) geopotential model EGM96, NASA/TP-1998206861. National Aeronautics and Space Administration, Maryland, USA. 1998b.

LEVISON, N. The Wiener RMS error criterion in filter design and prediction. Journal of Mathematical Physics. v. 25, p. 261-278, 1947.

LI, Y.; SIDERIS, M. Estimation and minimization of geoid undulation errors, In: International Association of Geodesy General Meeting. Beijing, China, 1993.

LOBIANCO, M.C.B. Determinação das alturas do geoide no Brasil. 2005. 165p. PhD Thesis - Escola Politécnica da Universidade de São Paulo, São Paulo, 2005. 
MATOS, A.C.O.C.; BLITZKOW, D. Modelagem Digital de Terrenos (MDT) de 3" para a América do Sul Pos-Doc Report - Escola Politécnica, Universidade de São Paulo, São Paulo, 2008.

MARTINEC, Z. Effect of lateral density variations of topographical masses in view of improving geoid model accuracy over Canada. Final Report of the contract DSS No. 23244-2-4356. Geodetic Survey of Canada, Ottawa, 1993.

MARTINEC, Z. Boundary-value problems for gravimetric determination of a precise geoid. Lecture Notes in Earth Sciences, v. 73, 1998.

MARTINEC, Z.; VANÍČEK, P. Direct topographical effect of Helmert's condensation for a spherical approximation of the geoid. Manuscripta Geodaetica, n. 19, p. 257268, 1994.

MAYER-GÜRR, T.; et al. The new combined satellite only model GOCO03s. International Symposium on Gravity, Geoid and Height Systems GGHS. October 2012.

MEISSL, P. Preparations for the numerical evaluation of second-order Molodenskii-type formulas. Columbus, Dep. of Geodetic Science and Surveying. Ohio State University, Report 163, 1971.

The use of finite elements in physical geodesy, OSU Rep 313, Dept Geod Sci Surv, Ohio State University, Columbus, 1981.

MIGLIACCIO, F.; et al. GOCE data analysis: the space-wise approach and the first space-wise gravity field model. Proceedings of the ESA Living Planet Symposium, 28 June - 2 July 2010, Bergen, Norway, 2010.

MIKHAIL, E.M.; AKERMANN, F. Observations and least square. New York: IEP-A Dun-Donnelley Publisher, 497 p. 1976.

MOLODENSKY, M.S.; EREMEV, V.F.; YURKINA, M.I. Methods for study of the external gravity field and figure of the earth. Israel Jerusalém Scientific Translations, Jerusalém, 1962.

MORITZ, H. Advanced physical geodesy, $2^{\text {nd }}$ Edition, Karlsruhe: Wichmann, 1989.

NOVÁK, P. Evaluation of gravity data for the Stokes-Helmert solution to the geodetic boundary-value problem. Technical Report No. 207, Department of Geodesy and Geomatics Engineering, University of New Brunswick, Fredericton. 2000.

PAIL R.; et al. GOCE gravity field model derived from orbit and gradiometry data applying the time-wise method Proceedings... of the ESA Living Planet Symposium, 28 June - 2 July 2010, Bergen, Norway, See also: earth.esa.int/GOCE. 2010a. 
PAIL R.; et al. Combined satellite gravity field model GOCO01S derived from GOCE and GRACE, Geophysical Research Letters, v. 37, L20314, doi:10.1029/2010GL044906, 2010b.

PAIL R.; et al. First GOCE gravity field models derived by three different approaches. Journal of Geodesy, v. 85, p. 819-843 DOI 10.1007/s00190-011-0467-x, 2011.

PAPOULIS, A. Signal analysis. McGraw-Hill, New York, 1977.

PAVLIS, N.K. Development and applications of the geopotential models. Lectures notes for the Second International School for he Determination and use of the Geoid. Rio de Janeiro, Brazil, 1997.

. Global gravitational modeling \& development and applications of geopotential models. (An overview considering current and future dedicated gravity mapping missions) Lectures notes for the Ninth International School for he Determination and use of the Geoid. La Plata, Argentina, 2010.

PAVLIS, N.K.; et al. An Earth gravitational model to degree 2160: EGM2008. presented at the 2008 General Assembly of the European Geosciences Union, Vienna, Austria, April 13-18, 2008.

PAVLIS, N.K.; et al. The development and evaluation of the Earth Gravitational Model 2008 (EGM2008), Journal of Geophysical Research, v. 117, p. 1-38, 2012.

RAPP, R.H. Geoid information by wavelength, Bulletin Géodésique, v. 110, n. 1, p. 405-411, 1973.

RAPP, R.H. Past and future developments in geopotential modeling, geodesy on the move. Forsberg, Feissel, Dietrich (eds), Springer-Verlag Berlin. New York, p. $58-78,1998$.

RAPP, R.H. ; RUMMEL, R. Methods for the computation of detailed geoids and their accuracy. OSU Rep 233, Dept Geod Sci Surv, Ohio State University, Columbus, 1975.

REIGBER, C.; et al. CHAMP phase-B executive summary. GFZ, STR96/13, 1996.

ROBBINS, J.W. Introduction to Hilbert space methods in physical geodesy. A term paper written for a course in Advanced Gravimetric Geodesy. 2008.

RUMMEL, R. Geoid and gravity in earth sciences-an overview. Workshop on Enabling Observation Techniques for Future Solid Earth Missions, Jan 30-Feb 01, 2003, Int. Space Sci. Inst. Bern Switzerland, Earth Moon and Planets n. 94, p. 3-11, 2005.

SÁ, N.C.; VIEIRA, C. Rede GPS do estado de São Paulo: aprimoramento de modelos geoidais e apoio básico local. Relatório científico final FAPESP (Processo: 99/012691-9) Instituto de Astronomia, Geofísica e Ciências Atmosféricas, Universidade de São Paulo, São Paulo, 90p, 2006. 
SALEH, J.; PAVLIS, N.K. The development and evaluation of the global digital terrain model DTM2002. Presented at the 3rd Meeting of the International Gravity and Geoid Commission, Thessaloniki, Greece 26 - 30 August 2002.

SCHWARZ, K.P.; SIDERIS, M.G.; FORSBERG, R. Orthometric heights without leveling. Journal of Surveying Engineering, v. 113, n. 1, p. 28-40, 1987.

. The use of FFT techniques in physical geodesy. Review Paper, Geophysical Journal International, v. 100, p 485-514, 1990.

SIDERIS, M.G. Geoid determination by FFT techniques. Lectures notes for the Ninth International School for he Determination and use of the Geoid. La Plata, Argentina, 2010.

SJÖBERG, L.E. Least-squares modification of Stokes's and Vening Meinesz's formula by accounting for truncation and potential coefficient errors. Manuscripta Geodaetica, v. 9, p. 209-229, 1984.

Refined least-squares modification of Stokes's formula. Manuscripta Geodaetica, v. 16, p. 367-375, 1991.

Quality estimates in Geoid Computation by EGM08. Journal of Geodetic Science, v. 1, n. 4, p. 361-366. 2011 Doi: 10.2478/v10156-011-0014-y.

SÜNKEL, H. Point mass models and the anomalous gravitational field, OSU Rep 328, Dept Geod Sci Surv, Ohio State University, Columbus, 1981.

SÜNKEL, $H$. The generation of mass point model from surface gravity data, OSU Rep 353, Dept Geod Sci Surv, Ohio State University, Columbus, 1983.

TAYLOR, A.E. Introduction to functional analysis. New York : John Wiley \& Sons, Inc. 423 p. 1958.

TORGE, W. Geodesy. $3^{\text {rd }}$ edition. Berlin; New York: de Gruyter. 2001.

TRENCH, W.F. An algorithm for the inverson of finite Toeplitz matrices. Journal of the Society for Industrial and Applied Mathematics, v. 12, n. 3, p. 515-522, 1964.

TSCHERNING C.C. Collocation methods in harmonic spaces. Proceedings... of the Free Boundary Value Problems Meeting. Born, 1971.

. Introduction to Functional Analysis with a View to its Application in Approximation Theory. In: Moritz, H. and H.Suenkel (Ed's): Approximation Methods in Geodesy, H.Wichmann Verlag, Karlsruhe, p. 157-192, 1978.

Computation of spherical harmonic coefficients and their error estimates using

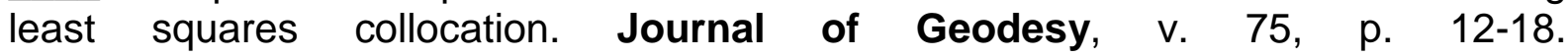
doi:10.1007/s001900000150, 2001. 
TSCHERNING C.C.; RAPP, R.H. Closed covariance expressions for gravity anomalies, geoid undulation and deflections of the vertical implied by the anomaly degree covariance models. Report of the Ohio State University. Columbus, n. 208, 1974

VANÍČEK, P.; KLEUSBERG, A. The Canadian geoid - Stokesian approach. Manuscripta Geodaetica, v. 12, p. 86-98, 1987.

VANIČEK P.; et al. The Canadian geoid. Technical Report $n^{\circ}$ 129. Dept. of Surveying Engineering-UNB. Fredericton, 1987.

VANÍČEK, P.; SJÖBERG, L.E. Reformulation of Stokes's theory for higher than second-degree reference field and modification of integration kernels, Journal of Geophysical Research, v. 96, p. 6529-6539, 1991.

VANÍČEK, P.; MARTINEC, Z. The Stokes-Helmert scheme for the evaluation of a precise geoid. Manuscripta Geodaetica, v. 19, p. 119-128, 1994.

VANÍČEK, P.; FEATHERSTONE, W.E. Performance of three types of Stoke's kernel in the combined solution for the geoid. Journal of Geodesy, Berlin v. 71, n. 12, p. 684-697, 1998.

VANÍČEK, P.; et al. Determination of the boundary values for the Stokes-Helmert problem. Journal of Geodesy, v. 73, p. 160-192, 1999.

VISSER, P.N.A.M.; VAN DEN IJSSEL, J; KOOP, R.; KLEES, R. Exploring gravity field determination from orbit perturbations of the European Gravity Mission GOCE. Journal of Geodesy, v. 75, p. 89-98, 2001.

WENZEL, H.G. Geoid computation by least-squares spectral combination using integration kernels. In: (ed) Proceedings... IAG General Meeting, Tokyo, p. 438-453, 1982.

WONG, L.; GORE, R. Accuracy of geoid heights from modified Stokes kernels, The Geophysical Journal of the Royal Astronomical Society, v. 18, p. 81-91, 1969. 